\title{
Afterglow Observations of Fermi Large Area Telescope Gamma-ray Bursts and the Emerging Class of Hyper-energetic Events
}

\section{Citation}

Cenko, S. B., D. A. Frail, F. A. Harrison, J. B. Haislip, D. E. Reichart, N. R. Butler, B. E. Cobb, et al. 2011. "AFTERGLOW OBSERVATIONS OFFERMILARGE AREA TELESCOPE GAMMA-RAY BURSTS AND THE EMERGING CLASS OF HYPER-ENERGETIC EVENTS." The Astrophysical Journal 732 (1): 29. https://doi.org/10.1088/0004-637x/732/1/29.

\section{Permanent link}

http://nrs.harvard.edu/urn-3:HUL.InstRepos:41397425

\section{Terms of Use}

This article was downloaded from Harvard University's DASH repository, and is made available under the terms and conditions applicable to Other Posted Material, as set forth at http:// nrs.harvard.edu/urn-3:HUL.InstRepos:dash.current.terms-of-use\#LAA

\section{Share Your Story}

The Harvard community has made this article openly available.

Please share how this access benefits you. Submit a story.

\section{Accessibility}




\title{
AFTERGLOW OBSERVATIONS OF FERMI LARGE AREA TELESCOPE GAMMA-RAY BURSTS AND THE EMERGING CLASS OF HYPER-ENERGETIC EVENTS
}

\author{
S. B. Cenko ${ }^{1}$, D. A. Frail ${ }^{2}$, F. A. Harrison ${ }^{3}$, J. B. Haislip ${ }^{4}$, D. E. Reichart ${ }^{4}$, N. R. Butler ${ }^{1,14}$, B. E. CobB ${ }^{1}$, \\ A. Cucchiara ${ }^{1,5}$, E. Berger ${ }^{6}$, J. S. Bloom ${ }^{1}$, P. Chandra $^{7}$, D. B. Fox ${ }^{5}$, D. A. Perley ${ }^{1}$, J. X. Prochaska ${ }^{8}$, \\ A. V. Filippenko ${ }^{1}$, K. Glazebrook ${ }^{9}$, K. M. Ivarsen $^{4}$, M. M. Kasliwal ${ }^{10}$, S. R. Kulkarni ${ }^{10}$, A. P. LaCluyze ${ }^{4}$, \\ S. Lopez ${ }^{11}$, A. N. Morgan ${ }^{1}$, M. Pettini ${ }^{12,13}$, And V. R. Rana ${ }^{3}$ \\ ${ }^{1}$ Department of Astronomy, University of California, Berkeley, CA 94720-3411, USA \\ 2 National Radio Astronomy Observatory, 1003 Lopezville Road, Socorro, NM 87801, USA \\ ${ }^{3}$ Space Radiation Laboratory, California Institute of Technology, MS 105-24, Pasadena, CA 91125 , USA \\ ${ }^{4}$ Department of Physics and Astronomy, University of North Carolina, Chapel Hill, NC 27599, USA \\ ${ }^{5}$ Department of Astronomy and Astrophysics, Pennsylvania State University, 525 Davey Laboratory, University Park, PA 16802, USA \\ ${ }^{6}$ Harvard-Smithsonian Center for Astrophysics, 60 Garden Street, Cambridge, MA 02138, USA \\ ${ }^{7}$ Department of Physics, Royal Military College of Canada, Kingston, ON, Canada \\ ${ }^{8}$ Department of Astronomy and Astrophysics, UCO/Lick Observatory, University of California, 1156 High Street, Santa Cruz, CA 95064, USA \\ ${ }^{9}$ Centre for Astrophysics and Supercomputing, Swinburne University of Technology, 1 Alfred Street, Hawthorn, Victoria 3122, Australia \\ ${ }^{10}$ Department of Astronomy, California Institute of Technology, MS 105-24, Pasadena, CA 91125, USA \\ ${ }^{11}$ Departamento de Astronomía, Universidad de Chile, Casilla 36-D, Santiago, Chile \\ ${ }^{12}$ Institute of Astronomy, Madingley Road, Cambridge CB3 OHA, UK \\ ${ }^{13}$ International Center for Radio Astronomy Research, University of Western Australia, 35 Stirling Hwy, Crawley, WA 6009, Australia \\ Received 2010 April 19; accepted 2011 February 16; published 2011 April 12
}

\begin{abstract}
We present broadband (radio, optical, and X-ray) light curves and spectra of the afterglows of four long-duration gamma-ray bursts (GRBs; GRBs 090323, 090328, 090902B, and 090926A) detected by the Gamma-Ray Burst Monitor and Large Area Telescope (LAT) instruments on the Fermi satellite. With its wide spectral bandpass, extending to GeV energies, Fermi is sensitive to GRBs with very large isotropic energy releases (10 $\left.{ }^{54} \mathrm{erg}\right)$. Although rare, these events are particularly important for testing GRB central-engine models. When combined with spectroscopic redshifts, our afterglow data for these four events are able to constrain jet collimation angles, the density structure of the circumburst medium, and both the true radiated energy release and the kinetic energy of the outflows. In agreement with our earlier work, we find that the relativistic energy budget of at least one of these events (GRB 090926A) exceeds the canonical value of $10^{51} \mathrm{erg}$ by an order of magnitude. Such energies pose a severe challenge for models in which the GRB is powered by a magnetar or a neutrino-driven collapsar, but remain compatible with theoretical expectations for magnetohydrodynamical collapsar models (e.g., the Blandford-Znajek mechanism). Our jet opening angles $(\theta)$ are similar to those found for pre-Fermi GRBs, but the large initial Lorentz factors $\left(\Gamma_{0}\right)$ inferred from the detection of $\mathrm{GeV}$ photons imply $\theta \Gamma_{0} \approx 70-90$, values which are above those predicted in magnetohydrodynamic models of jet acceleration. Finally, we find that these Fermi-LAT events preferentially occur in a low-density circumburst environment, and we speculate that this might result from the lower mass-loss rates of their lower-metallicity progenitor stars. Future studies of Fermi-LAT afterglows at radio wavelengths with the order-of-magnitude improvement in sensitivity offered by the Extended Very Large Array should definitively establish the relativistic energy budgets of these events.
\end{abstract}

Key words: cosmology: observations - gamma-ray burst: general - radio continuum: general

Online-only material: color figures

\section{INTRODUCTION}

Long-duration gamma-ray bursts $\left(\mathrm{GRBs}^{15}\right)$, like hydrogendeficient Type $\mathrm{Ib} / \mathrm{c}$ supernovae ( $\mathrm{SNe} \mathrm{Ib} / \mathrm{c}$; e.g., Filippenko 1997), result from the gravitational collapse of the evolved core of a massive star. The main characteristic that sets GRBs apart from other core-collapse $\mathrm{SNe}$ is that a substantial fraction of the energy of the explosion is coupled to relativistic ejecta. A compact central engine is responsible for accelerating and collimating these jet-like outflows and driving the SN explosions (Woosley \& Bloom 2006; Gehrels et al. 2009; Soderberg et al. 2010). The precise nature of the central engine which powers GRB-SNe, however, remains an open question.

\footnotetext{
${ }^{14}$ Einstein Fellow.

15 Throughout this work, we shall refer exclusively to long-duration GRBs (i.e., those apparently with massive-star progenitors; for a review, see Woosley \& Bloom 2006) unless explicitly stated otherwise.
}

Motivated by empirical constraints, all viable central-engine models for long-duration GRBs share some common characteristics (e.g., Piran 2005). They must produce a collimated outflow with an initial Lorentz factor $\left(\Gamma_{0}\right)$ of a few hundred on observed time scales of 10-100 s, with luminosities and kinetic energies of order $10^{50} \mathrm{erg} \mathrm{s}^{-1}$ and $10^{51} \mathrm{erg}$, respectively. Leading models include the "collapsar" model in which a relativistic jet is produced from a rotating black hole/accretion-disk system (Woosley 1993; MacFadyen \& Woosley 1999) and the "magnetar" model in which the rapid energy loss from a newly born millisecond neutron star with a $10^{15} \mathrm{G}$ magnetic field drives a Poynting-flux-dominated relativistic outflow (Usov 1992).

These and other more exotic models for GRB central engines are highly constrained by their energetics. The prompt highenergy emission, when combined with a spectroscopically determined redshift (and hence distance measurement), yields the isotropic radiated gamma-ray energy $\left(E_{\gamma, \text { iso }}\right)$. Well-sampled 


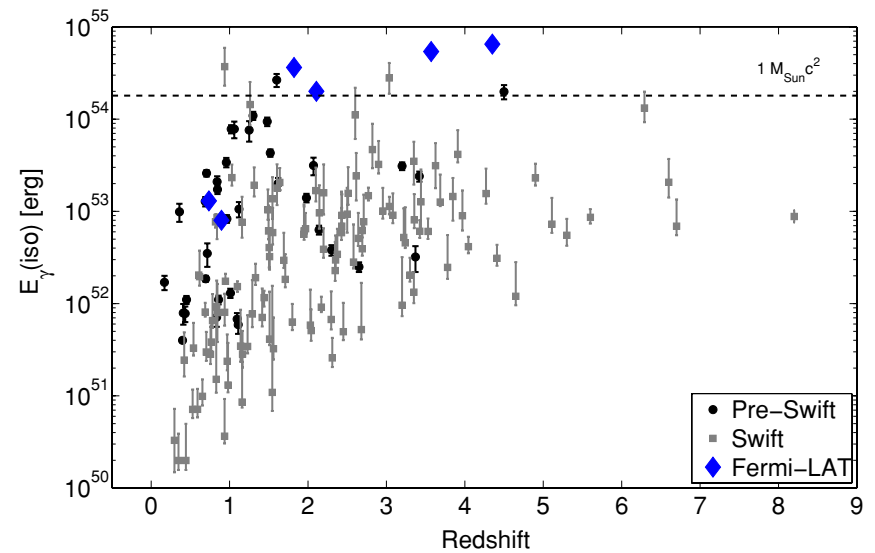

Figure 1. Prompt isotropic gamma-ray energy release $\left(E_{\gamma, \text { iso }}\right)$ of GRBs. With its soft, narrow bandpass (15-150 keV), Swift typically selects events having smaller isotropic energy release but larger opening angles than previous missions, which triggered predominantly in the MeV bandpass (Perna et al. 2003). GRBs detected at GeV energies with the Fermi-LAT all fall at the brightest end of the isotropic energy distribution, and must therefore be highly collimated to achieve a canonical beaming-corrected energy release of $\sim 10^{51}$ erg. References: pre-Swift: Amati 2006; Swift: Butler et al. 2007; FermiLAT: Greiner et al. 2009, this work.

(A color version of this figure is available in the online journal.)

afterglow observations allow both a measurement of the degree of collimation (and hence the true beaming-corrected energy release in the prompt emission, $E_{\gamma}$ ) and the kinetic energy remaining in the shock that powers the broadband afterglow emission $\left(E_{\mathrm{KE}}\right)$. Such measurements, made nearly a decade ago, pointed to a total relativistic energy yield $\left(E_{\mathrm{rel}} \approx E_{\gamma}+E_{\mathrm{KE}}\right)$ of $\sim 10^{51}$ erg (Frail et al. 2001; Panaitescu \& Kumar 2001a; Freedman \& Waxman 2001; Bloom et al. 2003; Berger et al. 2003a).

Since that time, there has been growing evidence for a considerable range in the relativistic energy scale $E_{\text {rel }}$, suggesting either a diversity in central engines or their properties. Most notably, a population of nearby (redshift $z \lesssim 0.1$ ), subenergetic GRBs has been identified (Bloom et al. 2003; Soderberg et al. 2004, 2006). They too are associated with $\mathrm{SNe} \mathrm{Ib} / \mathrm{c}$, but their relativistic energy release is a factor of 100 less than that of typical cosmological GRBs, and their outflows are significantly less collimated (quasi-spherical). Since they can only be detected at low redshifts where the comparative volume for discovery is low, they are small in total known number. But their volumetric rate is inferred to be 10-100 times larger than that of the more distant long-duration GRBs (Soderberg et al. 2006; Cobb et al. 2006; Liang et al. 2007).

More recently, evidence has been growing for a class of GRBs whose total relativistic energy release is at least an order of magnitude above the canonical value of $10^{51}$ erg (e.g., Cenko et al. 2010, and references therein). Unlike subluminous events, the total energy budget of these hyper-energetic events poses a significant challenge for some progenitor models. In particular, models in which the GRB is powered by a magnetar or a neutrino-driven collapsar are strongly disfavored. On the other hand, collapsars driven by magnetohydrodynamical (MHD) processes, such as the Blandford-Znajek mechanism (Blandford $\&$ Znajek 1977), can naturally accommodate energy budgets as large as $10^{53} \mathrm{erg}$.

Unfortunately, it has been rather difficult to constrain the beaming-corrected energetics for the hundreds of GRBs detected by the Swift satellite (Gehrels et al. 2004). The reasons for this difficulty are now largely understood. First, the relatively narrow energy bandpass $(15-150 \mathrm{keV})$ can miss entirely the peak of the gamma-ray spectrum, making estimates of $E_{\gamma, \text { iso }}$ highly uncertain. Second, there has been a dearth of measurements of jet opening angles (e.g., Panaitescu 2007; Kocevski \& Butler 2008; Liang et al. 2008; Racusin et al. 2009) and wellsampled multi-wavelength GRB afterglows (used to derive the afterglow kinetic energy $\left.E_{\mathrm{KE}}\right)$. Swift GRBs are on average more than twice as distant (Jakobsson et al. 2006) and therefore significantly fainter ( $\sim 1.5 \mathrm{mag}$ in the optical; Berger et al. 2005; Kann et al. 2010) than GRBs in previous samples. This is due in large part to selection effects: a combination of soft bandpass and high sensitivity from Swift has preferentially selected the faint end of the luminosity function-GRBs with low isotropic energy release but large opening angles (Perna et al. 2003).

With its nearly seven decades in energy coverage (10 keV-100 GeV), Fermi can provide unparalleled constraints on the spectral properties of the prompt emission. Furthermore, in light of the empirical relation between the peak energy of the gamma-ray spectrum and the isotropic gamma-ray energy release (the $E_{\mathrm{p}}-E_{\gamma, \text { iso }}$, or "Amati" relation; Amati 2006), MeV/ $\mathrm{GeV}$ events detected by either the Gamma-Ray Burst Monitor (GBM; $8 \mathrm{keV}-40 \mathrm{MeV}$; Meegan et al. 2009) or the Large Area Telescope (LAT; $20 \mathrm{MeV}-300 \mathrm{GeV}$; Atwood et al. 2009) on board Fermi preferentially select a sample of GRBs with large isotropic energy release (Figure 1). High- $E_{\gamma \text {,iso }}$ events also have brighter $\mathrm{X}$-ray and optical afterglows on average ( $\mathrm{Ny}$ sewander et al. 2009). Follow-up afterglow observations can then determine whether these GRBs are highly beamed events $\left(\theta \lesssim 2^{\circ}\right)$ with a typical energy release or true hyper-energetic GRBs.

The Fermi-LAT offers a further advantage over previous GRB missions sensitive only at $\mathrm{MeV}$ and $\mathrm{keV}$ energies by providing strict constraints on the initial Lorentz factor of the relativistic outflow. To avoid $e^{+}-e^{-}$pair production (and the accompanying thermal spectrum), the GRB jet must be moving toward the observer with ultra-relativistic speeds (the "compactness" problem; Cavallo \& Rees 1978). The higher the energy of the most energetic photon detected from a GRB, the stricter the lower limit on the outflow Lorentz factor will be. Combining the Lorentz factor limits for the most relativistic GRBs with inferred jet opening angles from broadband afterglow models can provide critical diagnostics of the jet acceleration mechanism.

Here we report on broadband (radio, optical, and X-ray) observations of four long-duration GRBs detected by the FermiLAT at GeV energies: GRBs 090323, 090328, 090902B, and 090926A. For each event we construct afterglow models to constrain the collimation and beaming-corrected energetics, and we compare these LAT events with previous GRBs detected at other energies (i.e., keV energies from Swift and $\mathrm{MeV}$ energies from pre-Swift satellites). For three of these GRBs, we also present the optical spectra used to determine the afterglow redshift. A more thorough analysis of the host-galaxy properties of these events will be presented in a forthcoming work.

Throughout this paper, we adopt a standard $\Lambda$ CDM cosmology with $H_{0}=71 \mathrm{~km} \mathrm{~s}^{-1} \mathrm{Mpc}^{-1}, \Omega_{\mathrm{m}}=0.27$, and $\Omega_{\Lambda}=$ $1-\Omega_{\mathrm{m}}=0.73$ (Spergel et al. 2007). We define the fluxdensity power-law temporal and spectral decay indices $\alpha$ and $\beta$ as $f_{v} \propto t^{-\alpha} v^{-\beta}$ (e.g., Sari et al. 1998). Optical magnitudes have been converted to flux densities using zero points from Fukugita et al. (1995). All quoted uncertainties are $1 \sigma(68 \%)$ confidence intervals unless otherwise noted. 


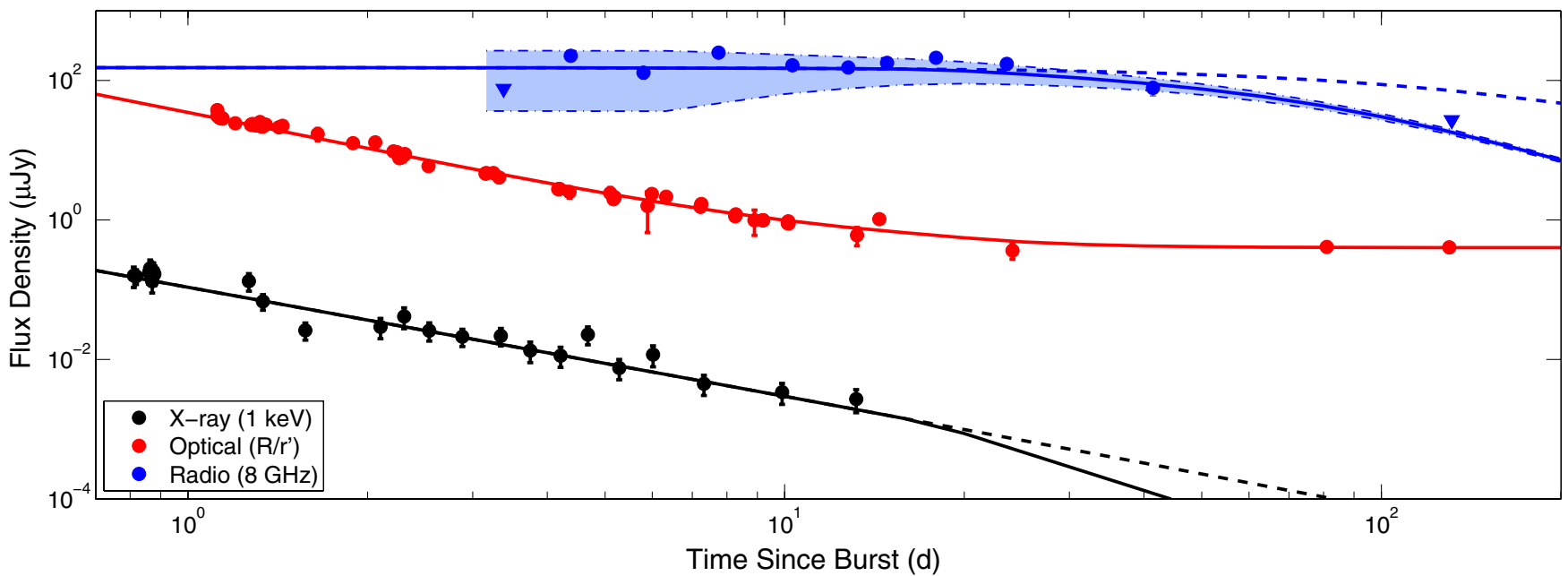

Figure 2. Broadband radio (blue), optical (red), and X-ray (black) light curves of GRB 090323. The best-fit model is plotted in solid lines (see Table 8 for parameters). The identical model parameters for an isotropic explosion are plotted as the dashed lines. The strength of the possible modulation of the radio afterglow caused by interstellar scintillation (e.g., Frail et al. 2000a) is indicated by the light-blue shaded region. The model provides a reasonable fit in all bandpasses. It is clear that any jet break must occur at $t>10$ days, although the upper bound on the jet break time is only weakly constrained.

(A color version of this figure is available in the online journal.)

\section{OBSERVATIONS}

\subsection{GRB 090323}

\subsubsection{High-energy Properties}

GRB 090323 was detected by the Fermi GBM at 00:02:42.63 on 2009 March 23 (Ohno et al. 2009; UT dates are used throughout this work). In the $8 \mathrm{keV}$ to $40 \mathrm{MeV}$ bandpass of the GBM, the light curve was multipeaked with a duration ${ }^{16}$ of $t_{90}=133.1 \pm 1.4 \mathrm{~s}$ (Bissaldi 2010). GRB 090323 was also detected at $\mathrm{MeV}$ energies by several of the satellites comprising the Inter-Planetary Network (IPN; Hurley et al. 1999), including the Konus instrument on the Wind satellite (Hurley et al. 2009; Golenetskii et al. 2009c).

Bissaldi (2010) analyzed the time-averaged spectrum of GRB 090323 (see also van der Horst 2009a), finding that the spectrum is best fit with a Band function (Band et al. 1993) having $\alpha=-1.05 \pm 0.02, \beta=-2.7_{-0.4}^{+0.2}$, and $E_{\mathrm{p}}=$ $591_{-33}^{+36} \mathrm{keV}$. The resulting fluence in the $8-10^{3} \mathrm{keV}$ (observer frame) bandpass is $f_{\gamma}=(1.22 \pm 0.01) \times 10^{-4} \mathrm{erg} \mathrm{cm}^{-2}$. The Konus-Wind instrument was able to measure the high-energy spectrum over the entire duration of the $\mathrm{MeV}$ emission. Fitting a Band model to their spectrum, Golenetskii et al. (2009c) find a reasonable fit with $\alpha=-0.96_{-0.09}^{+0.12}, \beta=-2.09_{-0.22}^{+0.16}$, $E_{\mathrm{p}}=416_{-73}^{+76} \mathrm{keV}$, and $f_{\gamma}=2.02_{-0.25}^{+0.28} \times 10^{-4} \mathrm{erg} \mathrm{cm}^{-2}$ (20-10 $\mathrm{keV}$ observer-frame bandpass).

In order to facilitate direct comparisons between events, we must transform this high-energy fluence into a common (restframe) bandpass (i.e., a " $k$ "-correction; Bloom et al. 2001). Here we adopt the rest-frame $1-10^{4} \mathrm{keV}$ bandpass, as this encompasses the full range of peak energies observed from GRBs (Band et al. 1993) without (for most pre-Fermi satellites) requiring large extrapolations outside the observed bandpass. At $z=3.568$ (Section 2.1.3), the GBM measurement corresponds to a prompt fluence of $f_{\gamma}=(1.24 \pm 0.05) \times 10^{-4} \mathrm{erg} \mathrm{cm}^{-2}$ in

\footnotetext{
16 It is customary to report GRB durations measured as the time between the arrival of $5 \%$ and $95 \%$ of the background-subtracted fluence. This quantity is referred to as $t_{90}$.
}

the $1-10^{4} \mathrm{keV}$ rest-frame bandpass (correcting the Konus-Wind spectrum yields a consistent result).

In addition to the detection at $\mathrm{MeV}$ energies by the GBM, GRB 090323 was detected at $\mathrm{GeV}$ energies by the Fermi LAT. Like several previous GRBs observed at $\mathrm{GeV}$ energies (e.g., Hurley et al. 1994; González et al. 2003; Giuliani et al. 2008; Abdo et al. 2009b), the GeV emission began several seconds after the $\mathrm{MeV}$ emission was detected, and remained significant long $(\sim 1000 \mathrm{~s})$ after the $\mathrm{MeV}$ component had faded beyond detectability (Ohno et al. 2009). The highest energy photon from the direction of GRB 090323 had an energy of $E \approx 7.5 \mathrm{GeV}$ (Piron et al. 2009). However, this photon arrived after the prompt $\mathrm{MeV}$ emission ended, and so its origin (i.e., prompt emission or afterglow, or, in physical terms, related to the internal or external shock) is somewhat uncertain. The highest energy photon detected by the LAT during the formal $\mathrm{MeV} t_{90}$ measurement had an energy of $E \approx 500 \mathrm{keV}$ (at $t \approx 90 \mathrm{~s}$; Piron et al. 2009).

\subsubsection{Afterglow Observations}

The Swift satellite began observations of the field of GRB 090323 with the onboard X-ray Telescope (XRT; Burrows et al. 2005b) at 19:27 on 2009 March $23(\sim 19.4 \mathrm{hr}$ after the burst; Kennea et al. 2009a). A candidate afterglow was promptly identified at $\alpha=12^{\mathrm{h}} 42^{\mathrm{m}} 50 \mathrm{~s} 26, \delta=$ $+17^{\circ} 03^{\prime} 14^{\prime \prime}$. 2 , with a $90 \%$ containment radius of $2^{\prime \prime} .7$ (J2000.0). The XRT continued to monitor the evolution of the fading counterpart for the following two weeks. We have obtained the XRT light curve from the online compilation of N.R.B.; ${ }^{17}$ the resulting evolution is plotted in Figure 2.

The optical afterglow of GRB 090323 was discovered shortly thereafter by GROND (Updike et al. 2009). The optical/nearinfrared (NIR) spectral energy distribution, constructed from simultaneous imaging in the $g^{\prime} r^{\prime} i^{\prime} z^{\prime} J H K$ filters, implied a spectral steepening around the observed $g^{\prime}$ band. Associating this break with absorption from Ly $\alpha$ in the GRB host galaxy, Updike et al. (2009) derive a photometric redshift of $4.0 \pm 0.3$ for

\footnotetext{
17 http://astro.berkeley.edu/ nat/swift; see Butler \& Kocevski (2007) for details.
} 
Table 1

Optical/NIR Observations of GRB 090323

\begin{tabular}{|c|c|c|c|c|c|c|}
\hline $\begin{array}{l}\text { Date }^{\mathrm{a}} \\
\text { (UT) }\end{array}$ & $\begin{array}{c}\text { Time Since Burst }{ }^{\mathrm{b}} \\
\text { (days) }\end{array}$ & Telescope/Instrument & Filter & $\begin{array}{c}\text { Exposure Time } \\
\text { (s) }\end{array}$ & Magnitude $^{c}$ & Reference $^{\mathrm{d}}$ \\
\hline 2009 Mar 24.19 & 1.20 & $\mathrm{P} 60$ & $r^{\prime}$ & 1800.0 & $20.50 \pm 0.07$ & $*$ \\
\hline 2009 Mar 24.20 & 1.21 & $\mathrm{P} 60$ & $i^{\prime}$ & 1800.0 & $19.95 \pm 0.04$ & $*$ \\
\hline 2009 Mar 24.23 & 1.23 & Gemini-S/GMOS & $i^{\prime}$ & 90.0 & $20.04 \pm 0.03$ & $*$ \\
\hline 2009 Mar 24.32 & 1.32 & $\mathrm{P} 60$ & $r^{\prime}$ & 900.0 & $20.46 \pm 0.04$ & $*$ \\
\hline 2009 Mar 24.33 & 1.33 & P60 & $i^{\prime}$ & 900.0 & $20.21 \pm 0.04$ & $*$ \\
\hline 2009 Mar 24.34 & 1.35 & P60 & $r^{\prime}$ & 900.0 & $20.56 \pm 0.04$ & $*$ \\
\hline 2009 Mar 24.36 & 1.36 & P60 & $i^{\prime}$ & 900.0 & $20.20 \pm 0.04$ & $*$ \\
\hline 2009 Mar 24.42 & 1.42 & $\mathrm{P} 60$ & $r^{\prime}$ & 900.0 & $20.65 \pm 0.05$ & $*$ \\
\hline 2009 Mar 24.43 & 1.43 & $\mathrm{P} 60$ & $i^{\prime}$ & 900.0 & $20.36 \pm 0.05$ & $*$ \\
\hline 2009 Mar 24.44 & 1.44 & P60 & $r^{\prime}$ & 900.0 & $20.60 \pm 0.04$ & $*$ \\
\hline 2009 Mar 24.45 & 1.46 & P60 & $i^{\prime}$ & 900.0 & $20.34 \pm 0.06$ & $*$ \\
\hline 2009 Mar 24.65 & 1.65 & Xinglong/TNT & $R$ & 6000.0 & 20.71 & 1 \\
\hline 2009 Mar 24.88 & 1.88 & TLS Tautenberg & $R$ & 4800.0 & $21.04 \pm 0.04$ & 2 \\
\hline 2009 Mar 25.06 & 2.06 & RTT150 & $R$ & 3600.0 & $21.01 \pm 0.04$ & 3 \\
\hline 2009 Mar 25.21 & 2.21 & P60 & $r^{\prime}$ & 720.0 & $21.51 \pm 0.10$ & $*$ \\
\hline 2009 Mar 25.22 & 2.22 & P60 & $i^{\prime}$ & 900.0 & $20.96 \pm 0.09$ & $*$ \\
\hline 2009 Mar 25.23 & 2.24 & P60 & $r^{\prime}$ & 900.0 & $21.54 \pm 0.11$ & $*$ \\
\hline 2009 Mar 25.24 & 2.25 & P60 & $i^{\prime}$ & 900.0 & $21.05 \pm 0.09$ & $*$ \\
\hline 2009 Mar 25.26 & 2.26 & P60 & $r^{\prime}$ & 720.0 & $21.76 \pm 0.09$ & $*$ \\
\hline 2009 Mar 25.27 & 2.27 & P60 & $i^{\prime}$ & 720.0 & $21.24 \pm 0.08$ & $*$ \\
\hline 2009 Mar 25.28 & 2.28 & P60 & $r^{\prime}$ & 720.0 & $21.72 \pm 0.08$ & $*$ \\
\hline 2009 Mar 25.29 & 2.29 & P60 & $i^{\prime}$ & 900.0 & $21.33 \pm 0.08$ & $*$ \\
\hline 2009 Mar 25.30 & 2.30 & Nickel & $R$ & 3300.0 & $21.46 \pm 0.2$ & 4 \\
\hline 2009 Mar 25.30 & 2.31 & P60 & $r^{\prime}$ & 720.0 & $21.61 \pm 0.07$ & $*$ \\
\hline 2009 Mar 25.31 & 2.32 & $\mathrm{P} 60$ & $i^{\prime}$ & 720.0 & $21.25 \pm 0.07$ & $*$ \\
\hline 2009 Mar 25.50 & 2.50 & Faulkes South & $i^{\prime}$ & 1800.0 & $21.3 \pm 0.2$ & 5 \\
\hline 2009 Mar 25.53 & 2.53 & Faulkes South & $R$ & 1800.0 & $21.7 \pm 0.1$ & 5 \\
\hline 2009 Mar 26.23 & 3.25 & P60 & $r^{\prime}$ & 3240.0 & $22.29 \pm 0.14$ & $*$ \\
\hline 2009 Mar 26.24 & 3.26 & P60 & $i^{\prime}$ & 2880.0 & $22.38 \pm 0.19$ & $*$ \\
\hline 2009 Mar 27.31 & 4.31 & P60 & $i^{\prime}$ & 5400.0 & $>22.60$ & $*$ \\
\hline 2009 Mar 27.36 & 4.36 & Nickel & $R$ & 15600.0 & $22.79 \pm 0.18$ & 6 \\
\hline 2009 Mar 28.11 & 5.10 & TLS Tautenberg & $R$ & 6600.0 & $22.83 \pm 0.20$ & 7 \\
\hline 2009 Mar 28.25 & 5.28 & $\mathrm{P} 60$ & $i^{\prime}$ & 4500.0 & $22.92 \pm 0.14$ & $*$ \\
\hline 2009 Mar 28.89 & 5.89 & TLS Tautenberg & $R$ & 2400.0 & $23.29 \pm 0.50$ & 8 \\
\hline 2009 Mar 29.00 & 5.99 & Shajn & $R$ & 5460.0 & $22.86 \pm 0.1$ & 9 \\
\hline 2009 Mar 29.32 & 6.33 & NOT & $R$ & 1800.0 & $22.96 \pm 0.06$ & 10 \\
\hline 2009 Mar 31.91 & 8.90 & TLS Tautenberg & $R$ & 7200.0 & $23.80 \pm 0.36$ & 8 \\
\hline 2009 Apr 6.40 & 14.41 & Gemini-N/GMOS & $r^{\prime}$ & 1200.0 & $23.94 \pm 0.10$ & $*$ \\
\hline 2009 Jul 29.96 & 129.97 & Gemini-S/GMOS & $r^{\prime}$ & 1800.0 & $24.95 \pm 0.15$ & $*$ \\
\hline
\end{tabular}

Notes.

${ }^{\text {a }}$ UT at the beginning of exposure.

b Time from midpoint of exposure to Fermi-GBM trigger.

${ }^{\mathrm{c}}$ Reported magnitudes have not been corrected for Galactic extinction ( $E(B-V)=0.025 \mathrm{mag}$; Schlegel et al. 1998). Observations in the $R$ band are referenced to Vega, while all other filters are reported on the AB magnitude system (Oke \& Gunn 1983).

d (*) This work; (1) Wang et al. 2009; (2) Kann et al. 2009b; (3) Burenin et al. 2009; (4) Perley et al. 2009a; (5) Guidorzi et al. 2009; (6) Perley 2009;

(7) Kann et al. 2009c; (8) Kann et al. 2009a; (9) Rumyantsev \& Pozanenko 2009; (10) de Ugarte Postigo et al. 2009.

GRB 090323. The full set of GROND observations is presented by McBreen et al. (2010); we include them in our modeling efforts, but for clarity they are omitted from Table 1.

We began observations of the afterglow of GRB 090323 with the automated Palomar 60 inch $(1.5 \mathrm{~m})$ telescope (P60; Cenko et al. 2006) beginning on 2009 March 24 (Cenko \& Perley 2009). Images were obtained in the Sloan $r^{\prime}$ and $i^{\prime}$ filters and individual frames were automatically reduced using our custom IRAF $^{18}$ software pipeline. To increase the signal-to-noise ratio $(\mathrm{S} / \mathrm{N})$, individual frames were astrometrically aligned using

\footnotetext{
18 IRAF is distributed by the National Optical Astronomy Observatory, which is operated by the Association of Universities for Research in Astronomy, Inc., under cooperative agreement with the National Science Foundation.
}

the Scamp software package and coadded using Swarp. ${ }^{19}$ We used aperture photometry to extract the flux of the afterglow from these coadded frames with the aperture radius roughly matched to the full width at half-maximum (FWHM) intensity of the point-spread function (PSF). Aperture magnitudes were then calibrated relative to field sources from the Sloan Digital Sky Survey (SDSS) Data Release 7 (Abazajian et al. 2009). Imaging continued on subsequent nights with the P60 until the afterglow was below our detection threshold. The results of this monitoring campaign, uncorrected for foreground Galactic extinction $(E[B-V]=0.025 \mathrm{mag}$; Schlegel et al. 1998), are presented in Table 1.

\footnotetext{
${ }^{19}$ See http://astromatic.iap.fr.
} 
Table 2

Radio Observations of GRB 090323

\begin{tabular}{lrccc}
\hline \hline $\begin{array}{c}\text { Date } \\
(\mathrm{UT})\end{array}$ & $\begin{array}{c}\Delta t \\
(\text { days })\end{array}$ & $\begin{array}{c}v \\
(\mathrm{GHz})\end{array}$ & $\begin{array}{c}f_{v} \\
(\mu \mathrm{Jy})\end{array}$ & $\begin{array}{c}\text { Facility } \\
\text { Mar 26.38 }\end{array}$ \\
Mar 27.38 & 4.38 & 8.46 & $27 \pm 38$ & VLA \\
Mar 27.99 & 4.99 & 8.46 & $225 \pm 35$ & VLA \\
Mar 28.43 & 5.43 & 8.9 & $105 \pm 24$ & WSRT \\
Mar 29.16 & 6.16 & 8.46 & $100 \pm 40$ & VLA \\
Mar 30.18 & 7.18 & 8.46 & $219 \pm 39$ & VLA \\
Mar 31.32 & 8.32 & 8.46 & $281 \pm 38$ & VLA \\
Apr 1.30 & 9.30 & 8.46 & $164 \pm 35$ & VLA \\
Apr 3.29 & 11.29 & 8.46 & $166 \pm 27$ & VLA \\
Apr 3.28 & 11.28 & 4.86 & $110 \pm 45$ & VLA \\
Apr 4.41 & 12.41 & 8.46 & $183 \pm 35$ & VLA \\
Apr 5.14 & 13.14 & 8.46 & $123 \pm 29$ & VLA \\
Apr 6.28 & 14.28 & 8.46 & $312 \pm 27$ & VLA \\
Apr 7.42 & 15.42 & 8.46 & $43 \pm 27$ & VLA \\
Apr 9.44 & 17.44 & 8.46 & $127 \pm 30$ & VLA \\
Apr 10.42 & 18.42 & 8.46 & $295 \pm 27$ & VLA \\
Apr 14.09 & 22.09 & 8.46 & $178 \pm 56$ & VLA \\
Apr 17.04 & 25.04 & 8.46 & $167 \pm 33$ & VLA \\
Apr 25.46 & 33.46 & 8.46 & $78 \pm 32$ & VLA \\
May 3.41 & 41.41 & 8.46 & $77 \pm 31$ & VLA \\
May 11.35 & 49.35 & 8.46 & $61 \pm 29$ & VLA \\
Aug 1.18 & 131.18 & 8.46 & $-13 \pm 27$ & VLA \\
\hline
\end{tabular}

Note. ${ }^{\text {a }}$ Reference: van der Horst (2009b).

We obtained additional imaging of the field of GRB 090323 with the two Gemini Multi-Object Spectrographs (GMOS-N and GMOS-S; Hook et al. 2004). Images were taken at both Gemini North and Gemini South in the Sloan $r^{\prime}$ and $i^{\prime}$ filters, and they were reduced using the IRAF gemini package. Photometry was performed with the same methodology used for the P60 imaging of the field, and the resulting measurements are shown in Table 1.

Additionally, we have compiled optical and NIR measurements of the afterglow of GRB 090323 from the GCN ${ }^{20}$ Circulars and included these in Table 1 . The majority of the late-time measurements (of most interest to us for modeling purposes) were obtained in the $R$ band. We note that most of these measurements were calibrated with respect to a single object (10700238439) from the USNO-B1 catalog (Monet et al. 2003), following Kann et al. (2009b). Using the SDSS observations of this field and the filter transformations of Jordi et al. (2006), we find an $R$-band magnitude for this source of $R=17.31 \mathrm{mag}$ (compared to an assumed value of 17.15 mag from Kann et al. 2009 b). We therefore offset all reported $R$-band magnitudes from the GCN Circulars by +0.16 mag.

Finally, we observed the afterglow of GRB 090323 with the Very Large Array ${ }^{21}$ (VLA) beginning a few days after the initial burst trigger. The flux-density scale was tied to 3C 286 or $3 \mathrm{C} 147$ and the phase was measured by switching between the GRB and a nearby bright point-source calibrator. To maximize sensitivity, the full VLA continuum bandwidth $(100 \mathrm{MHz})$ was recorded in two $50 \mathrm{MHz}$ bands. Data reduction was carried out following standard practice in the AIPS software package.

Our initial VLA detection of GRB 090323 was reported by Harrison et al. (2009). GRB 090323 was also detected at ra-

\footnotetext{
20 See http://gcn.gsfc.nasa.gov/gcn3_archive.html.

21 The Very Large Array is operated by the National Radio Astronomy Observatory, a facility of the National Science Foundation operated under cooperative agreement by Associated Universities, Inc.
}

dio wavelengths by the Westerbork Synthesis Radio Telescope (WSRT; van der Horst 2009b). The full set of VLA measurements is listed in Table 2. In order to improve the $\mathrm{S} / \mathrm{N}$ and to reduce the modulation of the light curve caused by interstellar scintillation (ISS; e.g., Frail et al. 2000a), we binned the data from adjacent epochs. These binned points were used for our afterglow modeling (Section 3 ) and are plotted in Figure 2.

\subsubsection{Optical Spectroscopy}

We began spectroscopic observations of the optical afterglow of GRB 090323 with GMOS-S on 2009 March 24 ( 29.93 hr after the GBM trigger; Chornock et al. 2009). We first obtained $2 \times 600$ s spectra with the B600 grating and a central wavelength of $6000 \AA$, providing coverage over the range $\sim 4500-7500 \AA$ with a resolution of $2.5 \AA$. Immediately following these exposures, we obtained $2 \times 600$ s spectra with the R400 grating and a central wavelength of $8000 \AA$, providing a coverage of $\sim 6000-10000 \AA$ A with a resolution of $5.0 \AA$.

The spectra were reduced in a standard manner using routines from the IRAF gemini and specred packages (see, e.g., Cenko et al. 2008 and references therein for details). Wavelength calibration was performed relative to $\mathrm{CuAr}$ lamps and then adjusted based on measured night-sky emission lines. The resulting root-mean-square wavelength uncertainty was $\lesssim 0.25 \AA$ for the spectra with the B600 grating and $\lesssim 0.40 \AA$ for the spectra taken with the R400 grating. Telluric features were removed using the continuum from well-exposed spectrophotometric standard stars (e.g., Wade \& Horne 1988; Matheson et al. 2000).

Flux calibration was performed relative to the spectrophotometric standard star LTT 7379 (Stone \& Baldwin 1983; Baldwin \& Stone 1984). We caution, however, that the standardstar observations were conducted on different nights from the GRB observations (2009 August 31 for the B600 grating; 2009 August 4 for the R400 grating), so the absolute flux calibration is somewhat uncertain (estimated to be $\sim 30 \%$ ).

The resulting spectrum of the afterglow of GRB 090323 is shown in Figure 3 . The broad absorption feature at $\lambda \approx 5570 \AA$ is produced by $\operatorname{Ly} \alpha$ at $z \approx 3.6$. The spectrum blueward of this wavelength is dominated by absorption from the Ly $\alpha$ forest, while the lack of Ly $\alpha$ forest features redward of this transition indicates that it corresponds to the redshift of the GRB host galaxy. Unfortunately, the GMOS CCD chip gap over the range 5500-5525 $\AA$ precludes an accurate measurement of the Ly $\alpha$ profile and hence a determination of the Hi column density.

Redward of $\mathrm{Ly} \alpha$, we find a series of strong (rest-frame equivalent width $W_{0} \geqslant 1 \AA$ ) absorption features superposed on a relatively flat, featureless continuum. In particular, we identify Si II $\lambda 1260, \mathrm{Si}_{\text {II}}^{*} \lambda 1264$, O I $\lambda 1302$, Si II $\lambda 1304$, Si II* $\lambda 1309$, C II $\lambda 1334$, II $^{*} \lambda 1335$, Si IV $\lambda \lambda 1393,1402$, Si II $\lambda 1526$, Si II* $\lambda 1533$, and $\mathrm{C}_{\mathrm{IV}} \lambda \lambda 1548,1550$ at $z=3.568 \pm 0.004$. Excited fine-structure lines are indicative of ultraviolet (UV) pumping and further suggest these features arise in the host galaxy of GRB 090323. The strongest features exhibit a complex velocity structure that is only marginally resolved with the B600 grating. We also detect intervening absorption systems of C IV $\lambda \lambda 1548,1550$ at $z=3.379$ and $\mathrm{Mg}$ II $\lambda \lambda 2796,2803$ at $z=2.101$. A more thorough analysis of the host-galaxy properties of GRB 090323 will be presented in a subsequent work.

At $z=3.568$, the isotropic prompt energy release from GRB 090323 in the rest-frame $1-10^{4} \mathrm{keV}$ bandpass is $E_{\gamma, \text { iso }}=$ 


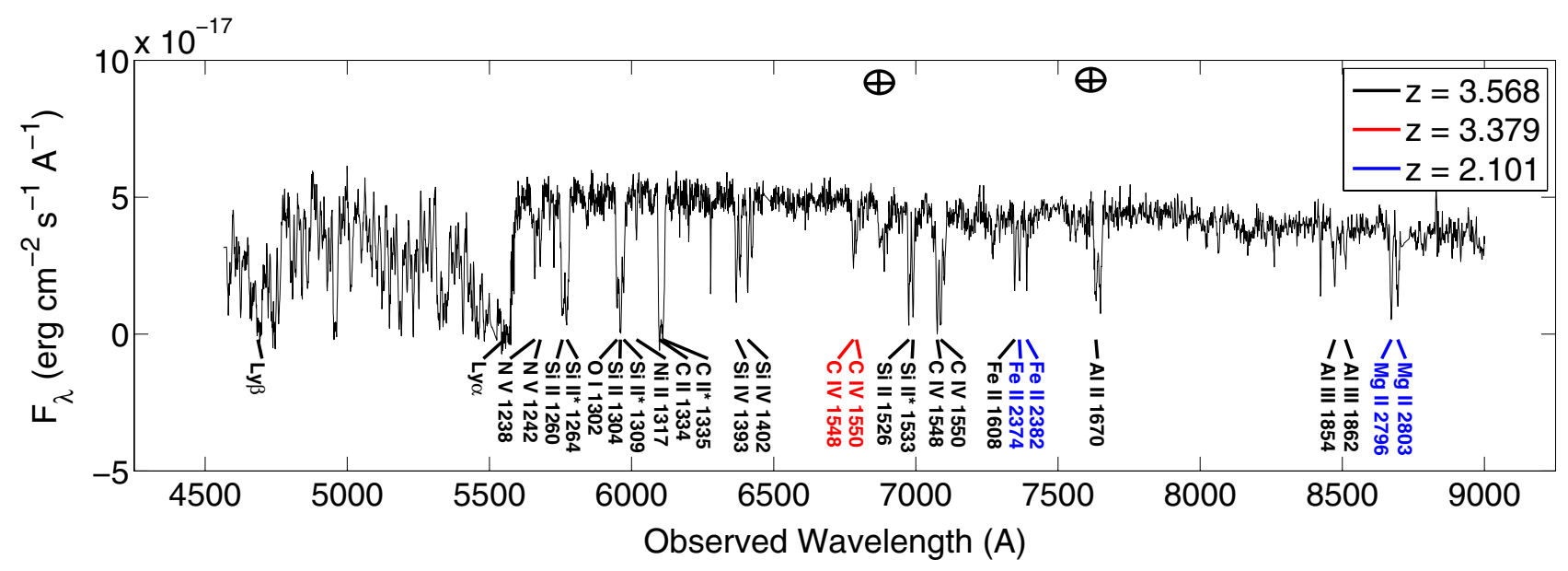

Figure 3. GMOS-S optical spectrum of the afterglow of GRB 090323. The broad absorption feature at $\lambda \approx 5570 \AA$ results from Ly $\alpha$ in the GRB host galaxy. We identify a number of strong, narrow absorption features redward of Ly $\alpha$ from the GRB host galaxy at a common redshift of $3.568 \pm 0.004$ (black annotations). The emission blueward of Ly $\alpha$ is strongly affected by the Ly $\alpha$ forest. We identify two additional intervening absorbers, based on Mg II $\lambda \lambda 2796,2803$ ( $z=2.101$; blue annotations) and C IV $\lambda \lambda 1548,1550(z=3.379$; red annotations). Areas of the spectrum affected by telluric absorption in the atmosphere of the Earth are marked with crossed circles. Because of the increased noise in the NIR due to fringing, we have cut off the plot at $\lambda=9000 \AA$.

(A color version of this figure is available in the online journal.)

$(3.30 \pm 0.13) \times 10^{54} \mathrm{erg}$. Using the formulation of Lithwick \& Sari (2001), the lower limit on the outflow Lorentz factor (assuming a nonthermal spectrum up to $E_{\text {obs }} \approx 500 \mathrm{keV}$ ) is $\Gamma_{0} \gtrsim 600$.

\subsection{GRB 090328}

\subsubsection{High-energy Properties}

GRB 090328 triggered the Fermi GBM at 09:36:46 on 2009 March 28 (McEnery et al. 2009). In the GBM bandpass, the light curve is multipeaked with a duration of $t_{90} \approx 57 \pm 3 \mathrm{~s}$ (Bissaldi 2010). GRB 090328 was also detected at $\mathrm{MeV}$ energies by several IPN satellites (Golenetskii et al. 2009a), including Konus-Wind (Golenetskii et al. 2009d).

The time-averaged GBM spectrum is well fit by a power law having an exponential cutoff, with $\alpha=-1.07 \pm 0.02$ and $E_{\mathrm{p}}=744_{-47}^{+50} \mathrm{keV}$ (Bissaldi 2010; see also Rau et al. 2009). The resulting fluence in the $8 \mathrm{keV}$ to $1 \mathrm{MeV}$ (observer frame) bandpass is $f_{\gamma}=(5.09 \pm 0.04) \times 10^{-5} \mathrm{erg} \mathrm{cm}^{-2}$. These results are all consistent with the analogous values derived by the Konus-Wind instrument. Given the wider bandpass of the GBM and the higher precision of its fluence measurement, we adopt this value for the remainder of this work. Using the observed redshift of 0.736 (Section 2.1.3), we find a prompt fluence of $f_{\gamma}=(6.80 \pm 0.7) \times 10^{-5} \mathrm{erg} \mathrm{cm}^{-2}$ in the $1-10^{4} \mathrm{keV}$ rest-frame bandpass.

GRB 090328 was also detected at GeV energies by the FermiLAT. Much like GRB 090323, flux in the LAT band from GRB 090328 is detected out to $\sim 900 \mathrm{~s}$ after the GBM trigger (Cutini et al. 2009). Many photons with energies above $1 \mathrm{GeV}$ are detected from the direction of GRB 090328; however, they are all detected well after the end of the $\mathrm{MeV}$ emission (Cutini et al. 2009; Piron et al. 2009). The highest energy photon detected from the direction of GRB 090328 was measured at $\sim 5 \mathrm{GeV}\left(t_{0}+798 \mathrm{~s}\right)$, while the most energetic photon detected during the prompt emission had $E \approx 700 \mathrm{keV}$ at $t_{0}+60 \mathrm{~s}$ (Piron et al. 2009).

\subsubsection{Afterglow Observations}

The Swift XRT began target-of-opportunity observations of GRB 090328 at 01:26 on 2009 March $29(\sim 15.9 \mathrm{hr}$ after the
GBM trigger). A fading X-ray source at $\alpha=6^{\mathrm{h}} 02^{\mathrm{m}} 39.58, \delta=$ $-41^{\circ} 52^{\prime} 57^{\prime \prime} .5$ (J2000.0; 2.'0 containment radius) was promptly identified as the X-ray afterglow (Kennea 2009; Kennea et al. $2009 b$ ). The resulting X-ray light curve, showing the afterglow evolution over the following 10 days, is plotted in Figure 4.

The Ultraviolet Optical Telescope (UVOT; Roming et al. 2005) onboard Swift began settled observations of the field of GRB 090328 approximately $16 \mathrm{hr}$ after the Fermi trigger. The optical afterglow, detected in both the $U$-band and white filters, was identified shortly thereafter (Oates 2009). UVOT observations of the afterglow continued for $\sim 2$ weeks, almost exclusively in these two filters. We have downloaded the UVOT $U$-band data from the HEASARC archive ${ }^{22}$ and conducted photometry with the images following the technique described by Li et al. (2006). Given the large uncertainty associated with flux calibration for the white filter, we have not included data taken in this band. The results of this analysis, not including a correction for foreground Galactic extinction $(E[B-V]=$ 0.057 mag; Schlegel et al. 1998), are shown in Table 3.

We obtained a single $i^{\prime}$ image of the afterglow of GRB 090328 with GMOS-S on 2009 April 29. This image was reduced in an identical manner to our observations of GRB 090323, and the zero point was calculated using predetermined values from the Gemini Web site. ${ }^{23}$ We have also included the simultaneous GROND optical and NIR measurements from McBreen et al. (2010) in our modeling, and therefore display them in Figure 4 as well (but not in Table 3).

Finally, we began observing the afterglow of GRB 090328 with the VLA on 2009 March 30 (Frail et al. 2009) and continued for nearly two months. The data were reduced in a manner identical to that described in Section 2.1.2. The results of our radio campaign are displayed in Table 4 (individual epochs) and plotted in Figure 4 (combined epochs).

\subsubsection{Optical Spectroscopy}

We began spectroscopic observations of GRB 090328 with GMOS-S at 00:05 on 2009 April 30 ( 38.5 hr after the GBM

\footnotetext{
22 See http://heasarc.gsfc.nasa.gov.

23 http://www.gemini.edu/sciops/instruments/ gmos/?q=sciops/instruments/gmos
} 


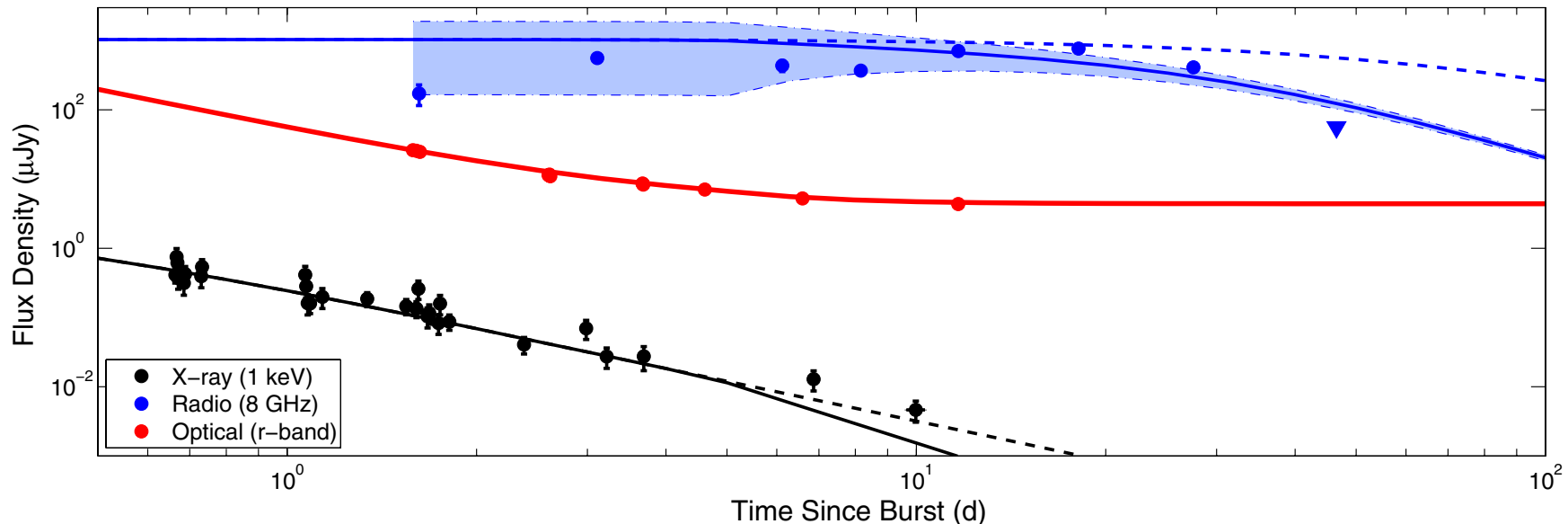

Figure 4. Broadband radio (blue), optical (red), and X-ray (black) light curves of GRB 090328. The best-fit model is plotted in solid lines (see Table 9 for parameters). The identical model parameters for an isotropic explosion are plotted as the dashed lines. The radio light curve is not very well fit at early times, although it likely suffers from strong interstellar scintillation (light-blue shaded region). In this case, a jet break is required by the data to fall at $t \gtrsim 10$ days.

(A color version of this figure is available in the online journal.)

Table 3

Optical Observations of GRB 090328

\begin{tabular}{|c|c|c|c|c|c|}
\hline $\begin{array}{l}\text { Date }^{\mathrm{a}} \\
\text { (UT) }\end{array}$ & $\begin{array}{c}\text { Time Since Burst } \\
\text { (days) }\end{array}$ & Telescope/Instrument & Filter & $\begin{array}{c}\text { Exposure Time } \\
(\mathrm{s})\end{array}$ & Magnitude $^{c}$ \\
\hline 2009 Mar 29.06 & 0.67 & UVOT & $U$ & 793.1 & $19.38 \pm 0.10$ \\
\hline 2009 Mar 29.13 & 0.73 & UVOT & $U$ & 462.1 & $19.58 \pm 0.16$ \\
\hline 2009 Mar 29.98 & 1.58 & Gemini-S/GMOS & $i^{\prime}$ & 150.0 & $19.98 \pm 0.10$ \\
\hline 2009 Mar 30.06 & 1.69 & UVOT & $U$ & 4111.2 & $20.80 \pm 0.11$ \\
\hline 2009 Mar 31.42 & 3.04 & UVOT & $U$ & 4162.2 & $21.96 \pm 0.20$ \\
\hline 2009 Apr 3.79 & 6.40 & UVOT & $U$ & 2094.3 & $21.52 \pm 0.24$ \\
\hline 2009 Apr 4.79 & 7.40 & UVOT & $U$ & 2507.4 & $21.69 \pm 0.23$ \\
\hline 2009 Apr 9.68 & 12.33 & UVOT & $U$ & 9493.6 & $22.67 \pm 0.24$ \\
\hline
\end{tabular}

Notes.

${ }^{\text {a }}$ UT at the beginning of exposure.

${ }^{\mathrm{b}}$ Time from midpoint of exposure to Fermi-GBM trigger.

${ }^{\mathrm{c}}$ Reported magnitudes have not been corrected for Galactic extinction $(E(B-V)=0.057 \mathrm{mag}$; Schlegel et al. 1998). Observations in the $U$ band are referenced to Vega, while all other filters are reported on the AB magnitude system (Oke \& Gunn 1983).

Table 4

VLA Radio Observations of GRB 090328

\begin{tabular}{cccc}
\hline \hline $\begin{array}{c}\text { Date } \\
(\mathrm{UT})\end{array}$ & $\begin{array}{c}\Delta t \\
(\text { days })\end{array}$ & $\begin{array}{c}v \\
(\mathrm{GHz})\end{array}$ & $\begin{array}{c}f_{v} \\
(\mu \mathrm{Jy})\end{array}$ \\
\hline Mar 30.02 & 1.62 & 8.46 & $172 \pm 57$ \\
Mar 30.99 & 2.59 & 8.46 & $337 \pm 60$ \\
Apr 1.02 & 3.62 & 8.46 & $783 \pm 57$ \\
Apr 3.03 & 5.63 & 8.46 & $195 \pm 34$ \\
Apr 4.01 & 6.61 & 8.46 & $674 \pm 155$ \\
Apr 5.09 & 7.69 & 8.46 & $214 \pm 41$ \\
Apr 6.02 & 8.62 & 8.46 & $523 \pm 57$ \\
Apr 6.97 & 9.57 & 8.46 & $809 \pm 39$ \\
Apr 11.14 & 13.74 & 8.46 & $603 \pm 67$ \\
Apr 14.00 & 16.60 & 8.46 & $643 \pm 59$ \\
Apr 16.99 & 19.59 & 8.46 & $886 \pm 58$ \\
Apr 24.97 & 27.57 & 8.46 & $410 \pm 59$ \\
May 9.96 & 42.56 & 8.46 & $78 \pm 43$ \\
May 11.86 & 44.46 & 8.46 & $-20 \pm 40$ \\
May 17.92 & 50.52 & & \\
\hline
\end{tabular}

trigger; Cenko et al. 2009). We obtained $2 \times 1500 \mathrm{~s}$ spectra with the R400 grating, the first with a central wavelength of $6000 \AA$ (providing a coverage of $\sim 4000-8000 \AA$ ) and the second with a central wavelength of $8000 \AA$ (providing a coverage of
6000-10000 $\AA$ ). The spectra were reduced in the manner described in Section 2.1.3. Flux calibration was performed relative to the standard star LTT 7379 taken with identical instrumental setups on 2009 August 4.

The resulting spectrum of GRB 090328 is shown in Figure 5. Superposed on a relatively flat continuum, we identify strong emission lines of oxygen ([O II $] \lambda 3727,[\mathrm{O}$ III $] \lambda \lambda 4959,5007$ ) as well as a series of narrow absorption features (Fe II $\lambda 2586$, Fe II $\lambda 2600, \mathrm{Mg}_{\text {II }} \lambda \lambda 2796,2803, \mathrm{Mg}_{\mathrm{I}} \lambda 2852, \mathrm{Ca}$ II $\lambda \lambda 3934$, $3969)$, all at $z=0.7357 \pm 0.0004$.

At this redshift, the prompt isotropic gamma-ray energy release from GRB 090328 in the $1-10^{4} \mathrm{keV}$ bandpass is $E_{\gamma, \text { iso }}=(9.6 \pm 1.0) \times 10^{52} \mathrm{erg}$. Using the formulation of Lithwick \& Sari (2001), the lower limit on the outflow Lorentz factor (assuming a nonthermal spectrum up to $E_{\mathrm{obs}} \approx 700 \mathrm{keV}$ ) is $\Gamma_{0} \gtrsim 200$.

\subsection{GRB 090902B}

\subsubsection{High-energy Properties}

At 11:05:08.31 on 2009 September 2, the Fermi-GBM triggered and located GRB 090902B (Bissaldi \& Connaughton 2009). In the GBM bandpass, the light curve consisted of a bright, multipeaked pulse with a duration $t_{90} \approx 21 \mathrm{~s}$. 


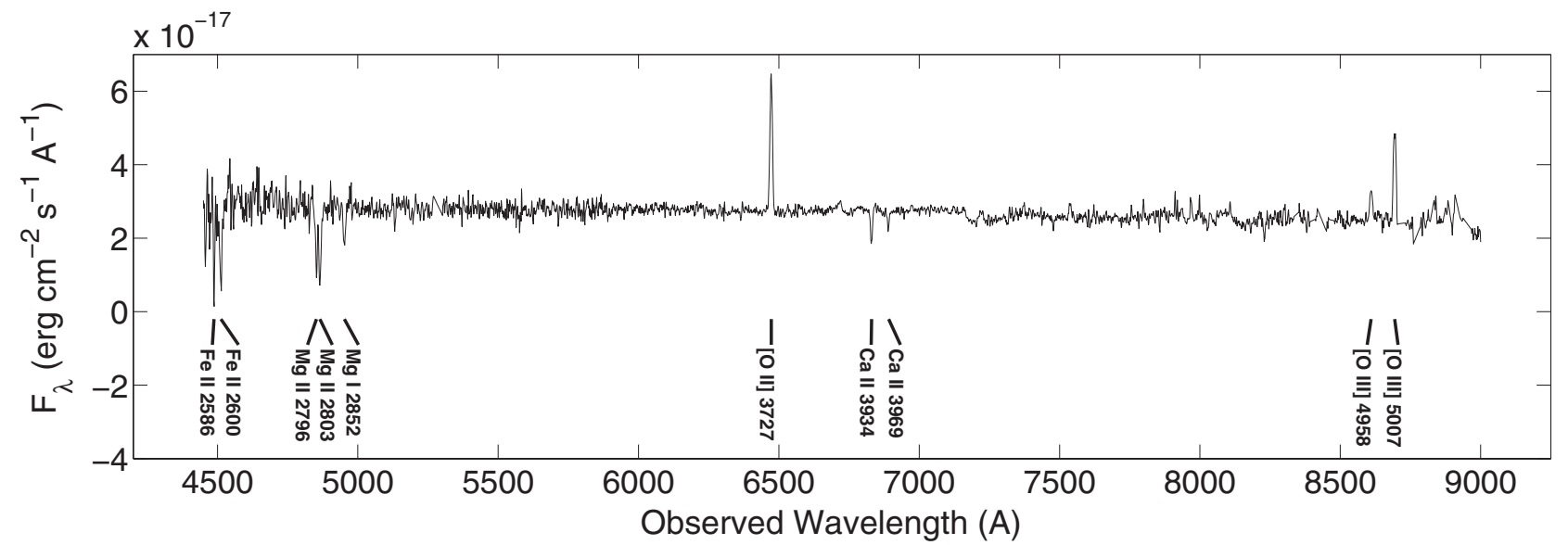

Figure 5. GMOS-S optical spectrum of the afterglow of GRB 090328. We identify a series of forbidden O emission lines and Fe, Mg, and Ca absorption features at a common redshift of $0.7357 \pm 0.0004$. We have only plotted the wavelength range $4500-9000 \AA$, due to the lower $\mathrm{S} / \mathrm{N}$ in the blue (spectrograph throughput) and the red (fringing).

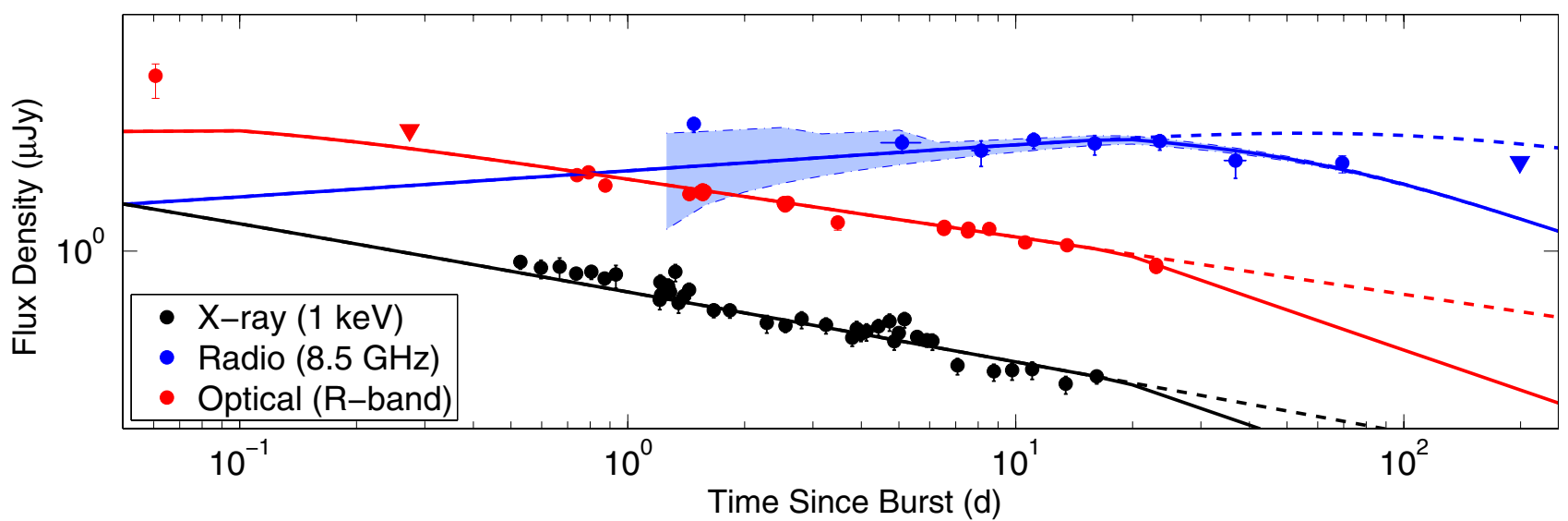

Figure 6. Broadband radio (blue), optical (red), and X-ray (black) light curves of GRB 090902B. The best-fit model is plotted in solid lines (see Table 10 for parameters). The identical model parameters for an isotropic explosion are plotted as the dashed lines. The strength of the possible modulation of the radio afterglow caused by interstellar scintillation (e.g., Frail et al. 2000a) is indicated by the light-blue shaded region. As suggested by Pandey et al. (2010), the early $(t \lesssim 0.3$ days) optical (and, to a lesser extent, radio) data are significantly larger than predicted by our forward-shock afterglow models. The most likely explanation is the presence of reverse-shock emission. The lack of a bright radio afterglow at late times suggests the presence of a jet break at $t \approx 20$ days.

(A color version of this figure is available in the online journal.)

GRB 090902B was also detected in a similar bandpass by Suzaku-WAM (Terada et al. 2009).

Furthermore, GRB 090902B was bright enough to be detected at $\mathrm{GeV}$ energies by the Fermi-LAT (de Palma et al. 2009). Like the other events in our sample, LAT emission was seen out to $t_{0}+1000 \mathrm{~s}$, at late times decaying like a power law with temporal index $\alpha_{\text {LAT }} \approx 1.5$ (Abdo et al. 2009a). GRB 090902B is responsible for the highest energy photon detected from a GRB to date, with $E=33.4_{-3.5}^{+3.7} \mathrm{GeV}$ at $t_{0}+82 \mathrm{~s}$. Within the $\mathrm{MeV}$ prompt emission phase, the highest energy photon was measured at $E=11.16_{-0.58}^{+1.48} \mathrm{GeV}$ (Abdo et al. 2009a).

Unlike the other events considered here, the prompt highenergy spectrum of GRB 090902B is not adequately described by a single Band function. While the $100-1000 \mathrm{keV}$ bandpass is reasonably well fit by such a model, the spectrum exhibits excess emission at both low $(\lesssim 100 \mathrm{keV})$ and high $(\gtrsim 10 \mathrm{MeV})$ energies. Abdo et al. (2009a) have suggested that the highenergy spectrum of GRB 090902B can be reproduced as the sum of two components: a Band function peaking at $\sim 700 \mathrm{keV}$, and a single power law (photon index $\Gamma \equiv \beta+1=1.93$ ) extending over the entire GBM+LAT bandpass. In this model, the powerlaw component accounts for $\sim 24 \%$ of the total $10 \mathrm{keV}$ to $10 \mathrm{GeV}$ fluence. The physical mechanism responsible for this complex spectrum is still not entirely understood; possible explanations include a hadronic origin (either proton synchrotron radiation (Razzaque et al. 2010) or photohadronic interactions (Asano et al. 2009)) or thermal emission from the jet photosphere (Mészáros \& Rees 2000; Ryde 2004; Ryde et al. 2010).

Using their two-component spectrum, Abdo et al. (2009a) report a total fluence of $f_{\gamma}=(4.59 \pm 0.05) \times 10^{-4} \mathrm{erg} \mathrm{cm}^{-2}$ in the $8 \mathrm{keV}$ to $30 \mathrm{GeV}$ (observer frame) bandpass. At a redshift of 1.8229 (Section 2.3.3), this corresponds to a prompt fluence of $(3.83 \pm 0.05) \times 10^{-4} \mathrm{erg} \mathrm{cm}^{-2}$ in the $1-10^{4} \mathrm{keV}$ rest-frame bandpass.

\subsubsection{Afterglow Observations}

The Swift XRT began target-of-opportunity observations of the field of GRB 090902B at 23:36 on 2009 September 2 $(\sim 12.5 \mathrm{hr}$ after the GBM trigger). A fading X-ray source at $\alpha=17^{\mathrm{h}} 39^{\mathrm{m}} 45^{\mathrm{s}} .26, \delta=+27^{\circ} 19^{\prime} 28^{\prime \prime} .1$ (J2000.0; $2^{\prime \prime} .1$ containment radius) was promptly identified as the X-ray afterglow (Kennea \& Stratta 2009; Evans 2009; Stratta et al. 2009). The resulting $\mathrm{X}$-ray light curve is shown in Figure 6.

The Swift UVOT began concurrently observing the field of GRB 090902B and first reported the detection of a candidate optical afterglow consistent with the X-ray position (Swenson 
Table 5

Optical Observations of GRB 090902B

\begin{tabular}{|c|c|c|c|c|c|}
\hline $\begin{array}{l}\text { Date }^{\mathrm{a}} \\
\text { (UT) }\end{array}$ & $\begin{array}{c}\text { Time Since Burst }{ }^{\mathrm{b}} \\
\text { (days) }\end{array}$ & Telescope/Instrument & Filter & $\begin{array}{c}\text { Exposure Time } \\
\text { (s) }\end{array}$ & Magnitude $^{\mathrm{c}}$ \\
\hline 2009 Sep 2.98 & 0.53 & UVOT & $U$ & 1074.7 & $20.31 \pm 0.15$ \\
\hline 2009 Sep 3.08 & 0.64 & UVOT & $U$ & 3612.9 & $20.54 \pm 0.09$ \\
\hline 2009 Sep 3.24 & 0.79 & Gemini-N/GMOS & $r^{\prime}$ & 180.0 & $20.68 \pm 0.15$ \\
\hline 2009 Sep 3.24 & 0.82 & UVOT & $U$ & 8756.0 & $20.92 \pm 0.08$ \\
\hline 2009 Sep 3.74 & 1.30 & UVOT & $U$ & 3852.8 & $22.06 \pm 0.26$ \\
\hline 2009 Sep 6.37 & 3.97 & UVOT & $U$ & 10549.1 & $22.25 \pm 0.21$ \\
\hline 2009 Sep 10.37 & 7.97 & UVOT & $U$ & 9969.0 & $>22.56$ \\
\hline
\end{tabular}

Notes.

${ }^{\text {a }} \mathrm{UT}$ at the beginning of exposure.

b Time from midpoint of exposure to Fermi-GBM trigger.

${ }^{\mathrm{c}}$ Reported magnitudes have not been corrected for Galactic extinction $(E(B-V)=0.042$ mag; Schlegel et al. 1998). Observations in the

$r^{\prime}$ filter are reported on the AB magnitude system (Oke \& Gunn 1983), while all other filters are referenced to Vega.

\& Siegel 2009). Subsequent observations revealed that the candidate had faded, confirming its association with GRB 090902B (Swenson \& Stratta 2009). In Table 5 we present UVOT $U$-band observations of GRB 090902B, reduced in a manner identical to that described in Section 2.2.2.

We obtained a single $r^{\prime}$ image of the afterglow of GRB 090902B with GMOS-N on 2009 September 3. This image was reduced in the same manner as those of the other events, and the resulting photometry is presented in Table 5. We have included optical and NIR photometry of GRB 090902B from Pandey et al. (2010) and McBreen et al. (2010) in our modeling, and therefore display them in Figure 6 as well.

Finally, we began observing the afterglow of GRB 090902B with the VLA on 2009 September 3 (Chandra \& Frail 2009) and continued for $\sim 5$ months. The data were reduced as described in Section 2.2.2. The results of our radio campaign are displayed in Table 6 (individual epochs) and plotted in Figure 6 (combined epochs). We have also included the early radio detection at $4.8 \mathrm{GHz}$ reported by the WSRT (van der Horst et al. 2009) in Table 6.

\subsubsection{Optical Spectroscopy}

We began spectroscopic observations of GRB 090902B with GMOS-N at 06:29 on 2009 September $3(\sim 19.4 \mathrm{hr}$ after the GBM trigger; Cucchiara et al. 2009). We obtained $2 \times 900 \mathrm{~s}$ exposures, both with the R400 grating and a central wavelength of $6000 \AA$, providing a coverage of $\sim 4000-8000 \AA$. The spectra were reduced as described in Sections 2.1.3 and 2.2.3. Flux calibration was performed relative to spectra of the standard star Feige 34 (Oke 1990) taken with the same instrumental setup on 2008 May 1.

The resulting spectrum of GRB 090902B is shown in Figure 7. Superimposed on a smooth power-law continuum, we identify strong absorption features corresponding to $\mathrm{Mg}_{\mathrm{I}} \lambda 2852$, Mg II $\lambda \lambda 2796,2803$, Mn II $\lambda 2606$, Fe II $\lambda 2600$, Mn II $\lambda 2594$, Fe II $\lambda 2586$, Mn II $\lambda 2576$, Fe I $\lambda 2484$, Fe II $\lambda 2382$, Fe II $\lambda 2344$, Fe II $\lambda 2260$, Fe I $\lambda 2167$, Cr II $\lambda \lambda 2056,2062$, and Mg I $\lambda 2026$, all at a common redshift of $1.8229 \pm 0.0004$.

The lack of evidence for Ly $\alpha$ absorption down to $\lambda \lesssim 4000 \AA$ implies an upper bound on the redshift of the GRB host galaxy of $z \lesssim 2.3$. Together with the strength of the above features (in particular Fe I 22167), we consider it quite likely that the observed system at $z=1.8229$ derives from the GRB host galaxy.
Table 6

Radio Observations of GRB 090902B

\begin{tabular}{ccccc}
\hline \hline $\begin{array}{c}\text { Date } \\
(\mathrm{UT})\end{array}$ & $\begin{array}{c}\Delta t \\
(\text { days })\end{array}$ & $\begin{array}{c}v \\
(\mathrm{GHz})\end{array}$ & $\begin{array}{c}f_{v} \\
(\mu \mathrm{Jy})\end{array}$ & Facility \\
\hline 2009 Sep 3.77 & 1.31 & 4.8 & $111 \pm 28$ & WSRT \\
2009 Sep 3.94 & 1.48 & 8.46 & $141 \pm 39$ & VLA \\
2009 Sep 7.05 & 4.59 & 8.46 & $13 \pm 31$ & VLA \\
2009 Sep 8.05 & 5.59 & 8.46 & $130 \pm 34$ & VLA \\
2009 Sep 10.15 & 7.69 & 8.46 & $10 \pm 32$ & VLA \\
2009 Sep 11.05 & 8.59 & 8.46 & $80 \pm 32$ & VLA \\
2009 Sep 13.10 & 10.64 & 8.46 & $99 \pm 31$ & VLA \\
2009 Sep 14.14 & 11.60 & 8.46 & $71 \pm 33$ & VLA \\
2009 Sep 18.04 & 15.50 & 8.46 & $52 \pm 32$ & VLA \\
2009 Sep 19.00 & 16.46 & 8.46 & $89 \pm 36$ & VLA \\
2009 Sep 25.09 & 22.51 & 8.46 & $26 \pm 29$ & VLA \\
2009 Sep 27.08 & 24.50 & 8.46 & $67 \pm 29$ & VLA \\
2009 Oct 7.01 & 34.43 & 8.46 & $38 \pm 28$ & VLA \\
2009 Oct 9.01 & 36.43 & 8.46 & $66 \pm 27$ & VLA \\
2009 Oct 11.81 & 39.23 & 8.46 & $21 \pm 31$ & VLA \\
2009 Nov 6.90 & 65.44 & 8.46 & $9 \pm 20$ & VLA \\
2009 Nov 7.77 & 66.31 & 8.46 & $22 \pm 19$ & VLA \\
2009 Nov 9.00 & 67.54 & 8.46 & $48 \pm 19$ & VLA \\
2009 Nov 14.88 & 73.42 & 8.46 & $31 \pm 21$ & VLA \\
2010 Mar 20.62 & 199.16 & 8.46 & $18 \pm 16$ & VLA \\
\hline
\end{tabular}

Note. ${ }^{\text {a }}$ Reference: van der Horst et al. (2009).

At this redshift, the prompt isotropic gamma-ray energy release in the $1-10^{4} \mathrm{keV}$ rest-frame bandpass is $E_{\gamma \text {,iso }}=$ $(3.20 \pm 0.04) \times 10^{54} \mathrm{erg}$. Assuming a nonthermal spectrum up to $E_{\text {obs }}=11 \mathrm{GeV}$, Abdo et al. (2009a) have determined a lower limit to the initial outflow Lorentz factor of $\Gamma_{0} \gtrsim 1000$.

\subsection{GRB 090926A}

\subsubsection{High-energy Properties}

GRB 090926A triggered the GBM on Fermi at 04:20:26.99 on 2009 September 26 (Bissaldi 2009). The light curve consists of a single pulse with duration $t_{90} \approx 20 \mathrm{~s}$ in the GBM bandpass. The prompt emission was sufficiently bright to trigger several additional satellite instruments, including Suzaku-WAM (Noda et al. 2009), Konus-Wind (Golenetskii et al. 2009b), and RT-2 on CORONAS-PHOTON (Chakrabarti et al. 2009).

Simultaneously fitting both the GBM and LAT (see below) data from $t_{0}$ to $t_{0}+21 \mathrm{~s}$, Bissaldi et al. (2009) report that the spectrum is reasonably well modeled by a Band function 


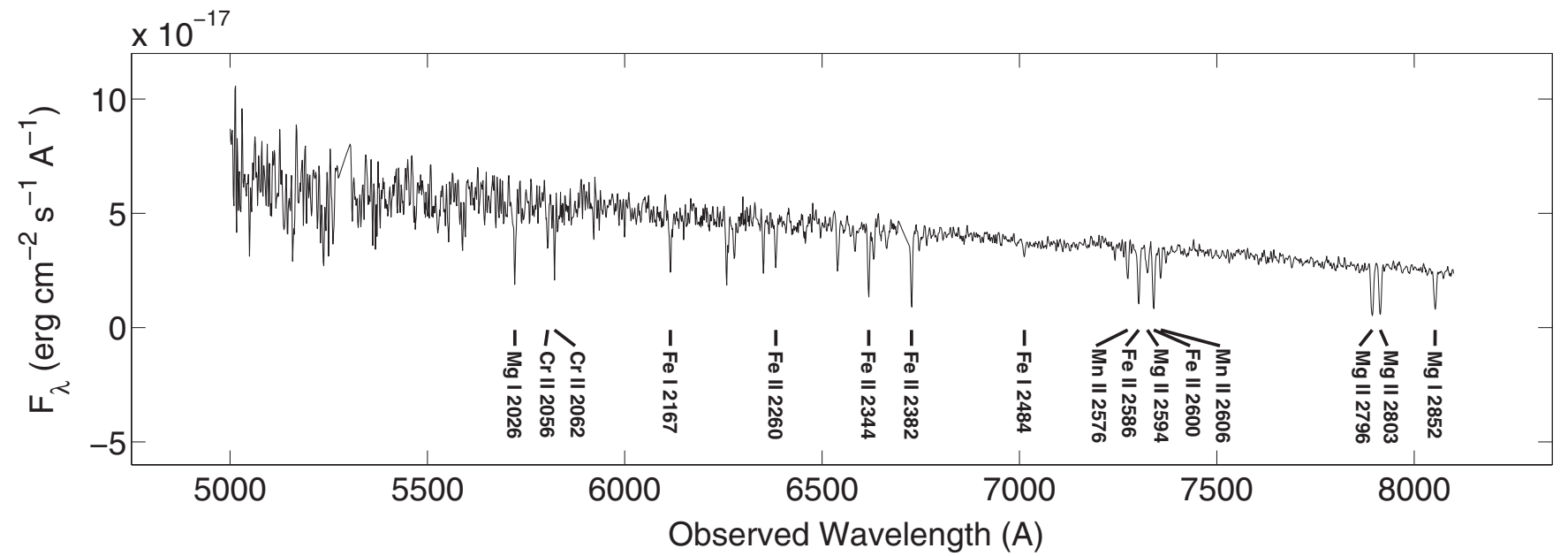

Figure 7. GMOS-N optical spectrum of the afterglow of GRB 090902B. We identify a series of absorption features from neutral and singly ionized Mg, Mn, Fe, and $\mathrm{Cr}$ at a common redshift of $1.8229 \pm 0.0004$. We have not plotted the shortest wavelengths due to the decreased spectrograph throughput.

with $\alpha=-0.693 \pm 0.009, \beta=-2.342 \pm 0.011$, and $E_{\mathrm{p}}=$ $268 \pm 4 \mathrm{keV}$. The corresponding $10 \mathrm{keV}$ to $10 \mathrm{GeV}$ fluence (observer frame) is $f_{\gamma}=(2.47 \pm 0.03) \times 10^{-4} \mathrm{erg} \mathrm{cm} \mathrm{cm}^{-2}$. These results differ only slightly from the values reported from other satellites. At $z=2.1062$ (Malesani et al. 2009), the corresponding prompt fluence in the rest-frame $1-10^{4} \mathrm{keV}$ bandpass is $(1.73 \pm 0.03) \times 10^{-4} \mathrm{erg} \mathrm{cm}^{-2}$.

GRB 090926A was also detected by the Fermi-LAT. The emission in the LAT bandpass lasted at least $400 \mathrm{~s}$, with possible indications of significant flux out to $\sim 1000 \mathrm{~s}$ after the GBM trigger. Many photons with $E>1 \mathrm{GeV}$ were detected from the position of GRB 090926A, with the highest energy measured of $20 \mathrm{GeV}$ at $t_{0}+26 \mathrm{~s}$ (Uehara et al. 2009).

\subsubsection{Afterglow Observations}

Swift XRT began observing the field of GRB 090926A at $17: 17$ on 2009 September $26(\sim 13 \mathrm{hr}$ after the GBM trigger). A fading X-ray counterpart at $\alpha=23^{\mathrm{h}} 33^{\mathrm{m}} 36^{\mathrm{s}} .18$, $\delta=-66^{\circ} 19^{\prime} 25^{\prime \prime} .9$ (J2000.0, $1^{\prime \prime} .5$ containment radius) was promptly identified in the XRT data (Vetere et al. 2009; Vetere 2009). The XRT observed GRB 090926A for the next 3 weeks, and we plot the X-ray light curve in Figures 8 and 9.

Under the control of Skynet, four of the 16 inch diameter PROMPT telescopes (Reichart et al. 2005) at the Cerro Tololo Inter-American Observatory (CTIO) observed the Fermi-LAT localization of GRB 090926 A beginning $19.0 \mathrm{hr}$ after the GBM trigger in the $B-, V-, R$-, and $I$-band filters. Within the Swift-XRT localization, we identified an uncataloged and fading source as the optical afterglow of GRB 090926A (Haislip et al. 2009b, 2009a). Individual images were automatically reduced using our custom, IRAF-based reduction pipeline and then astrometrically aligned and stacked. We subsequently measured the afterglow flux with aperture photometry, where the inclusion radius was approximately matched to the FWHM of the PSF. PROMPT continued to observe the field for 10 more nights (Haislip et al. 2009e, 2009c, 2009d).

We also obtained three epochs of $I$ - and $J$-band imaging of the afterglow of GRB 090926A using the ANDICAM (A Novel Dual Imaging CAMera) instrument mounted on the $1.3 \mathrm{~m}$ telescope at CTIO. ${ }^{24}$ This telescope is operated as part of the Small and Moderate Aperture Research Telescope System (SMARTS)

\footnotetext{
24 http://www.astronomy.ohio-state.edu/ANDICAM
}

consortium. ${ }^{25}$ Each epoch consisted of six individual $360 \mathrm{~s}$ $I$-band observations and 30 individual $60 \mathrm{~s} J$-band observations. Between optical exposures, the telescope was slightly offset and the individual $J$-band exposures were additionally dithered via an internal tilting mirror system. Standard data reduction was performed on these images, including cosmic ray rejection, overscan bias subtraction, zero subtraction, flat fielding, and sky subtraction to correct for the NIR background and the $I$-band fringing. For each epoch, the individual images were then aligned and averaged to produce a single frame in each band with summed exposure times of 36 minutes in $I$ and 30 minutes in $J$.

Relative aperture photometry was performed on the SMARTS data, in comparison with a number of nonvariable sources in the field of GRB 090926A. The I-band field was photometrically calibrated by comparison (on photometric nights) with Landolt standard stars in the field of $\mathrm{T}$ Phe (Landolt 1992). J-band photometric calibration was performed using Two Micron All Sky Survey (Skrutskie et al. 2006) field stars.

SMARTS BVRI observations of the field of GRB 090926A were also obtained on two photometric nights a few months after the GRB occurred (2009 December 16 and 18). For these observations, total summed exposure times amounted to $180 \mathrm{~s}$ in $B R I$ and $120 \mathrm{~s}$ in $V$. The absolute photometry of the field was again established based on same-night observations of the $T$ Phe Landolt standard stars. These observations were then used to provide absolute calibration for the PROMPT observations of GRB 090926A.

We acquired additional late-time images of the field of GRB 090926A on 2009 October 19 with GMOS-S on Gemini South. A total of $600 \mathrm{~s}$ of exposure time was obtained in the Sloan $g^{\prime}-, r^{\prime}$-, and $i^{\prime}$-band filters. The data were reduced in the manner described in Section 2.1.2 and calibrated in the same way as the PROMPT and SMARTS observations.

The results of our optical and NIR monitoring campaign of the afterglow of GRB 090926A, uncorrected for the modest amount of Galactic extinction $(E(B-V)=0.024 \mathrm{mag}$; Schlegel et al. 1998), are shown in Table 7 and Figures 8 and 9. Also included in Figures 8 and 9 are the optical and NIR observations presented by Rau et al. (2010).

\footnotetext{
$25 \mathrm{http}: / / \mathrm{www} \cdot a$ astro.yale.edu/smarts
} 
Table 7

Optical/NIR Observations of GRB 090926A

\begin{tabular}{|c|c|c|c|c|c|}
\hline $\begin{array}{l}\text { Date }^{\mathrm{a}} \\
\text { (UT) }\end{array}$ & $\begin{array}{c}\text { Time Since Burst }{ }^{\mathrm{b}} \\
\text { (days) }\end{array}$ & Telescope/Instrument & Filter & $\begin{array}{c}\text { Exposure Time } \\
\text { (s) }\end{array}$ & Magnitude $^{c}$ \\
\hline 2009 Sep 26.72 & 0.5415 & UVOT & $U$ & 358.0 & $18.33 \pm 0.06$ \\
\hline 2009 Sep 26.72 & 0.5501 & UVOT & $V$ & 358.0 & $18.34 \pm 0.10$ \\
\hline 2009 Sep 26.78 & 0.5989 & UVOT & $U$ & 675.0 & $18.50 \pm 0.05$ \\
\hline 2009 Sep 26.79 & 0.6149 & UVOT & $V$ & 675.0 & $18.91 \pm 0.09$ \\
\hline 2009 Sep 26.84 & 0.6649 & UVOT & $U$ & 704.5 & $18.71 \pm 0.05$ \\
\hline 2009 Sep 26.85 & 0.6816 & UVOT & $V$ & 703.3 & $19.09 \pm 0.10$ \\
\hline 2009 Sep 26.90 & 0.7299 & UVOT & $U$ & 526.0 & $18.84 \pm 0.07$ \\
\hline 2009 Sep 26.96 & 0.7966 & PROMPT-2 & $V$ & 560.0 & $18.49 \pm 0.22$ \\
\hline 2009 Sep 26.96 & 0.7969 & PROMPT-4 & $R$ & 640.0 & $18.80 \pm 0.16$ \\
\hline 2009 Sep 26.96 & 0.7986 & PROMPT-5 & $I$ & 400.0 & $18.13 \pm 0.19$ \\
\hline 2009 Sep 26.98 & 0.8062 & UVOT & $U$ & 459.4 & $18.94 \pm 0.08$ \\
\hline 2009 Sep 26.99 & 0.8172 & UVOT & $V$ & 459.4 & $18.97 \pm 0.12$ \\
\hline 2009 Sep 27.00 & 0.8250 & PROMPT-5 & $I$ & 720.0 & $18.23 \pm 0.07$ \\
\hline 2009 Sep 27.00 & 0.8259 & PROMPT-4 & $R$ & 880.0 & $18.60 \pm 0.05$ \\
\hline 2009 Sep 27.00 & 0.8314 & PROMPT-2 & V & 1600.0 & $18.85 \pm 0.10$ \\
\hline 2009 Sep 27.00 & 0.8372 & PROMPT-5 & $I$ & 880.0 & $18.17 \pm 0.05$ \\
\hline 2009 Sep 27.00 & 0.8379 & PROMPT-4 & $R$ & 880.0 & $18.56 \pm 0.04$ \\
\hline 2009 Sep 27.00 & 0.8553 & PROMPT-4 & $R$ & 800.0 & $18.52 \pm 0.04$ \\
\hline 2009 Sep 27.00 & 0.8553 & PROMPT-5 & $I$ & 800.0 & $18.15 \pm 0.05$ \\
\hline 2009 Sep 27.00 & 0.8583 & SMARTS/ANDICAM & $I$ & 2160.0 & $18.17 \pm 0.07$ \\
\hline 2009 Sep 27.00 & 0.8583 & SMARTS/ANDICAM & $J$ & 1800.0 & $17.28 \pm 0.07$ \\
\hline 2009 Sep 27.00 & 0.8603 & PROMPT-2 & $V$ & 1520.0 & $18.85 \pm 0.09$ \\
\hline 2009 Sep 27.04 & 0.8654 & PROMPT-3 & $B$ & 880.0 & $19.11 \pm 0.10$ \\
\hline 2009 Sep 27.04 & 0.8659 & PROMPT-4 & $R$ & 800.0 & $18.47 \pm 0.04$ \\
\hline 2009 Sep 27.04 & 0.8660 & PROMPT-5 & $I$ & 800.0 & $18.08 \pm 0.05$ \\
\hline 2009 Sep 27.04 & 0.8662 & UVOT & $U$ & 689.8 & $18.80 \pm 0.06$ \\
\hline 2009 Sep 27.04 & 0.8761 & PROMPT-4 & $R$ & 800.0 & $18.47 \pm 0.04$ \\
\hline 2009 Sep 27.04 & 0.8764 & PROMPT-5 & $I$ & 640.0 & $18.06 \pm 0.05$ \\
\hline 2009 Sep 27.04 & 0.8808 & PROMPT-2 & $V$ & 1520.0 & $18.92 \pm 0.09$ \\
\hline 2009 Sep 27.04 & 0.8811 & PROMPT-3 & $B$ & 1520.0 & $19.12 \pm 0.07$ \\
\hline 2009 Sep 27.05 & 0.8826 & UVOT & V & 689.7 & $18.86 \pm 0.09$ \\
\hline 2009 Sep 27.04 & 0.8858 & PROMPT-5 & $I$ & 800.0 & $17.98 \pm 0.04$ \\
\hline 2009 Sep 27.04 & 0.8864 & PROMPT-4 & $R$ & 800.0 & $18.42 \pm 0.04$ \\
\hline 2009 Sep 27.04 & 0.8962 & PROMPT-5 & $I$ & 800.0 & $18.08 \pm 0.05$ \\
\hline 2009 Sep 27.04 & 0.8967 & PROMPT-4 & $R$ & 800.0 & $18.40 \pm 0.04$ \\
\hline 2009 Sep 27.04 & 0.9012 & PROMPT-3 & $B$ & 1600.0 & $19.11 \pm 0.07$ \\
\hline 2009 Sep 27.04 & 0.9013 & PROMPT-2 & V & 1520.0 & $18.75 \pm 0.08$ \\
\hline 2009 Sep 27.08 & 0.9055 & PROMPT-5 & $I$ & 640.0 & $17.96 \pm 0.05$ \\
\hline 2009 Sep 27.08 & 0.9065 & PROMPT-4 & $R$ & 720.0 & $18.45 \pm 0.04$ \\
\hline 2009 Sep 27.08 & 0.9172 & PROMPT-4 & $R$ & 880.0 & $18.40 \pm 0.04$ \\
\hline 2009 Sep 27.08 & 0.9172 & PROMPT-5 & $I$ & 880.0 & $17.83 \pm 0.03$ \\
\hline 2009 Sep 27.08 & 0.9223 & PROMPT-3 & $B$ & 1680.0 & $18.93 \pm 0.05$ \\
\hline 2009 Sep 27.08 & 0.9225 & PROMPT-2 & $V$ & 1600.0 & $18.68 \pm 0.07$ \\
\hline 2009 Sep 27.08 & 0.9280 & PROMPT-4 & $R$ & 800.0 & $18.40 \pm 0.04$ \\
\hline 2009 Sep 27.08 & 0.9284 & PROMPT-5 & $I$ & 560.0 & $17.96 \pm 0.05$ \\
\hline 2009 Sep 27.10 & 0.9331 & UVOT & $U$ & 691.7 & $18.64 \pm 0.05$ \\
\hline 2009 Sep 27.08 & 0.9388 & PROMPT-4 & $R$ & 880.0 & $18.26 \pm 0.03$ \\
\hline 2009 Sep 27.08 & 0.9388 & PROMPT-5 & $I$ & 880.0 & $17.87 \pm 0.04$ \\
\hline 2009 Sep 27.08 & 0.9419 & PROMPT-3 & $B$ & 1360.0 & $19.11 \pm 0.08$ \\
\hline 2009 Sep 27.08 & 0.9421 & PROMPT-2 & $V$ & 1280.0 & $18.58 \pm 0.08$ \\
\hline 2009 Sep 27.13 & 0.9475 & PROMPT-4 & $R$ & 480.0 & $18.36 \pm 0.05$ \\
\hline 2009 Sep 27.13 & 0.9477 & PROMPT-5 & $I$ & 480.0 & $17.93 \pm 0.05$ \\
\hline 2009 Sep 27.12 & 0.9495 & UVOT & $V$ & 691.7 & $18.76 \pm 0.09$ \\
\hline 2009 Sep 27.17 & 0.9933 & PROMPT-3 & $B$ & 960.0 & $19.15 \pm 0.07$ \\
\hline 2009 Sep 27.17 & 0.9933 & PROMPT-5 & $I$ & 960.0 & $17.93 \pm 0.04$ \\
\hline 2009 Sep 27.17 & 0.9935 & PROMPT-2 & V & 960.0 & $18.61 \pm 0.10$ \\
\hline 2009 Sep 27.17 & 0.9938 & PROMPT-4 & $R$ & 1040.0 & $18.32 \pm 0.03$ \\
\hline 2009 Sep 27.17 & 0.9992 & UVOT & $U$ & 554.0 & $18.65 \pm 0.06$ \\
\hline 2009 Sep 27.17 & 1.0058 & PROMPT-5 & $I$ & 960.0 & $17.94 \pm 0.03$ \\
\hline 2009 Sep 27.17 & 1.0062 & PROMPT-4 & $R$ & 880.0 & $18.35 \pm 0.03$ \\
\hline 2009 Sep 27.17 & 1.0100 & PROMPT-3 & $B$ & 1200.0 & $18.98 \pm 0.06$ \\
\hline 2009 Sep 27.17 & 1.0107 & PROMPT-2 & V & 1520.0 & $18.76 \pm 0.09$ \\
\hline 2009 Sep 27.17 & 1.0166 & PROMPT-4 & $R$ & 560.0 & $18.30 \pm 0.04$ \\
\hline 2009 Sep 27.17 & 1.0173 & PROMPT-5 & $I$ & 640.0 & $17.90 \pm 0.04$ \\
\hline
\end{tabular}


Table 7

(Continued)

\begin{tabular}{|c|c|c|c|c|c|}
\hline $\begin{array}{l}\text { Date }^{\mathrm{a}} \\
\text { (UT) }\end{array}$ & $\begin{array}{c}\text { Time Since Burst }{ }^{\mathrm{b}} \\
\text { (days) }\end{array}$ & Telescope/Instrument & Filter & $\begin{array}{c}\text { Exposure Time } \\
\text { (s) }\end{array}$ & Magnitude $^{c}$ \\
\hline 2009 Sep 27.21 & 1.0293 & PROMPT-4 & $R$ & 960.0 & $18.37 \pm 0.03$ \\
\hline 2009 Sep 27.21 & 1.0295 & PROMPT-5 & $I$ & 880.0 & $17.96 \pm 0.04$ \\
\hline 2009 Sep 27.21 & 1.0352 & PROMPT-2 & $V$ & 1680.0 & $18.62 \pm 0.07$ \\
\hline 2009 Sep 27.21 & 1.0363 & PROMPT-3 & $B$ & 1360.0 & $19.08 \pm 0.07$ \\
\hline 2009 Sep 27.21 & 1.0408 & PROMPT-5 & $I$ & 800.0 & $17.98 \pm 0.04$ \\
\hline 2009 Sep 27.21 & 1.0412 & PROMPT-4 & $R$ & 880.0 & $18.54 \pm 0.04$ \\
\hline 2009 Sep 27.21 & 1.0530 & PROMPT-5 & $I$ & 960.0 & $18.12 \pm 0.04$ \\
\hline 2009 Sep 27.21 & 1.0530 & PROMPT-4 & $R$ & 960.0 & $18.53 \pm 0.04$ \\
\hline 2009 Sep 27.21 & 1.0590 & PROMPT-2 & $V$ & 1840.0 & $18.86 \pm 0.10$ \\
\hline 2009 Sep 27.21 & 1.0592 & PROMPT-3 & $B$ & 1920.0 & $19.19 \pm 0.06$ \\
\hline 2009 Sep 27.21 & 1.0653 & PROMPT-4 & $R$ & 960.0 & $18.54 \pm 0.04$ \\
\hline 2009 Sep 27.21 & 1.0655 & PROMPT-5 & $I$ & 960.0 & $18.11 \pm 0.05$ \\
\hline 2009 Sep 27.24 & 1.0658 & UVOT & $U$ & 730.1 & $18.67 \pm 0.05$ \\
\hline 2009 Sep 27.25 & 1.0777 & PROMPT-4 & $R$ & 960.0 & $18.67 \pm 0.04$ \\
\hline 2009 Sep 27.25 & 1.0780 & PROMPT-5 & $I$ & 960.0 & $18.13 \pm 0.04$ \\
\hline 2009 Sep 27.25 & 1.0839 & PROMPT-3 & $B$ & 1920.0 & $19.14 \pm 0.05$ \\
\hline 2009 Sep 27.25 & 1.0839 & PROMPT-2 & V & 1840.0 & $18.88 \pm 0.09$ \\
\hline 2009 Sep 27.25 & 1.0900 & PROMPT-4 & $R$ & 960.0 & $18.56 \pm 0.03$ \\
\hline 2009 Sep 27.25 & 1.0902 & PROMPT-5 & $I$ & 880.0 & $18.08 \pm 0.04$ \\
\hline 2009 Sep 27.25 & 1.1028 & PROMPT-5 & $I$ & 1040.0 & $18.09 \pm 0.04$ \\
\hline 2009 Sep 27.25 & 1.1029 & PROMPT-4 & $R$ & 1040.0 & $18.50 \pm 0.03$ \\
\hline 2009 Sep 27.25 & 1.1091 & PROMPT-3 & $B$ & 2000.0 & $19.06 \pm 0.04$ \\
\hline 2009 Sep 27.25 & 1.1093 & PROMPT-2 & V & 1920.0 & $18.69 \pm 0.07$ \\
\hline 2009 Sep 27.29 & 1.1158 & PROMPT-4 & $R$ & 960.0 & $18.58 \pm 0.03$ \\
\hline 2009 Sep 27.29 & 1.1158 & PROMPT-5 & $I$ & 960.0 & $18.15 \pm 0.04$ \\
\hline 2009 Sep 27.29 & 1.1283 & PROMPT-5 & $I$ & 960.0 & $18.13 \pm 0.04$ \\
\hline 2009 Sep 27.29 & 1.1287 & PROMPT-4 & $R$ & 1040.0 & $18.56 \pm 0.03$ \\
\hline 2009 Sep 27.29 & 1.1348 & PROMPT-3 & $B$ & 2000.0 & $19.17 \pm 0.05$ \\
\hline 2009 Sep 27.29 & 1.1352 & PROMPT-2 & V & 1920.0 & $18.82 \pm 0.09$ \\
\hline 2009 Sep 27.29 & 1.1413 & PROMPT-5 & $I$ & 1040.0 & $18.08 \pm 0.04$ \\
\hline 2009 Sep 27.29 & 1.1415 & PROMPT-4 & $R$ & 960.0 & $18.54 \pm 0.03$ \\
\hline 2009 Sep 27.33 & 1.1545 & PROMPT-4 & $R$ & 960.0 & $18.58 \pm 0.03$ \\
\hline 2009 Sep 27.33 & 1.1550 & PROMPT-5 & $I$ & 1040.0 & $18.17 \pm 0.05$ \\
\hline 2009 Sep 27.33 & 1.1617 & PROMPT-2 & V & 2000.0 & $18.84 \pm 0.07$ \\
\hline 2009 Sep 27.33 & 1.1617 & PROMPT-3 & $B$ & 2080.0 & $19.22 \pm 0.06$ \\
\hline 2009 Sep 27.33 & 1.1684 & PROMPT-4 & $R$ & 1040.0 & $18.62 \pm 0.03$ \\
\hline 2009 Sep 27.33 & 1.1685 & PROMPT-5 & $I$ & 1040.0 & $18.17 \pm 0.05$ \\
\hline 2009 Sep 27.33 & 1.1818 & PROMPT-4 & $R$ & 1040.0 & $18.63 \pm 0.04$ \\
\hline 2009 Sep 27.33 & 1.1826 & PROMPT-5 & $I$ & 960.0 & $18.35 \pm 0.06$ \\
\hline 2009 Sep 27.33 & 1.1850 & PROMPT-3 & $B$ & 1520.0 & $19.29 \pm 0.08$ \\
\hline 2009 Sep 27.33 & 1.1859 & PROMPT-2 & $V$ & 1600.0 & $18.78 \pm 0.10$ \\
\hline 2009 Sep 27.33 & 1.1919 & PROMPT-4 & $R$ & 480.0 & $18.74 \pm 0.07$ \\
\hline 2009 Sep 27.33 & 1.1923 & PROMPT-5 & $I$ & 480.0 & $18.39 \pm 0.11$ \\
\hline 2009 Sep 27.38 & 1.2093 & PROMPT-3 & $B$ & 480.0 & $19.28 \pm 0.19$ \\
\hline 2009 Sep 27.38 & 1.2093 & PROMPT-4 & $R$ & 480.0 & $18.82 \pm 0.08$ \\
\hline 2009 Sep 27.38 & 1.2093 & PROMPT-5 & $I$ & 480.0 & $18.29 \pm 0.10$ \\
\hline 2009 Sep 27.38 & 1.2095 & PROMPT-2 & $V$ & 480.0 & $18.70 \pm 0.23$ \\
\hline 2009 Sep 27.84 & 1.6702 & UVOT & $U$ & 663.2 & $19.67 \pm 0.10$ \\
\hline 2009 Sep 27.91 & 1.7349 & UVOT & $U$ & 735.0 & $19.74 \pm 0.09$ \\
\hline 2009 Sep 27.95 & 1.7846 & UVOT & $V$ & 2168.5 & $19.82 \pm 0.09$ \\
\hline 2009 Sep 27.97 & 1.8018 & UVOT & $U$ & 737.0 & $19.71 \pm 0.09$ \\
\hline 2009 Sep 28.04 & 1.8685 & UVOT & $U$ & 742.9 & $19.78 \pm 0.10$ \\
\hline 2009 Sep 28.11 & 1.9344 & UVOT & $U$ & 577.3 & $19.79 \pm 0.11$ \\
\hline 2009 Sep 28.12 & 1.9476 & UVOT & $U$ & 341.3 & $19.73 \pm 0.14$ \\
\hline 2009 Sep 28.40 & 2.2343 & UVOT & $U$ & 1329.2 & $20.13 \pm 0.08$ \\
\hline 2009 Sep 28.55 & 2.3829 & UVOT & $U$ & 1730.2 & $20.01 \pm 0.07$ \\
\hline 2009 Sep 28.56 & 2.3950 & UVOT & $V$ & 1131.6 & $20.07 \pm 0.14$ \\
\hline 2009 Sep 28.88 & 2.7199 & UVOT & $U$ & 2642.7 & $20.11 \pm 0.06$ \\
\hline 2009 Sep 28.89 & 2.7277 & UVOT & $V$ & 2417.0 & $20.11 \pm 0.10$ \\
\hline 2009 Sep 29.08 & 2.9301 & PROMPT-5 & $I$ & 3200.0 & $19.59 \pm 0.10$ \\
\hline 2009 Sep 29.08 & 2.9346 & PROMPT-4 & $R$ & 2560.0 & $20.07 \pm 0.12$ \\
\hline 2009 Sep 29.13 & 2.9642 & PROMPT-5 & $I$ & 1840.0 & $19.50 \pm 0.11$ \\
\hline 2009 Sep 29.13 & 2.9644 & PROMPT-4 & $R$ & 1840.0 & $19.99 \pm 0.12$ \\
\hline 2009 Sep 29.16 & 2.9892 & SMARTS/ANDICAM & $I$ & 2160.0 & $19.53 \pm 0.07$ \\
\hline
\end{tabular}


Table 7

(Continued)

\begin{tabular}{|c|c|c|c|c|c|}
\hline $\begin{array}{l}\text { Date }^{\mathrm{a}} \\
\text { (UT) }\end{array}$ & $\begin{array}{c}\text { Time Since Burst }{ }^{\mathrm{b}} \\
\text { (days) }\end{array}$ & Telescope/Instrument & Filter & $\begin{array}{c}\text { Exposure Time } \\
\text { (s) }\end{array}$ & Magnitude $^{c}$ \\
\hline 2009 Sep 29.16 & 2.9892 & SMARTS/ANDICAM & $J$ & 1800.0 & $18.35 \pm 0.09$ \\
\hline 2009 Sep 29.17 & 3.0041 & PROMPT-5 & $I$ & 2720.0 & $19.58 \pm 0.10$ \\
\hline 2009 Sep 29.17 & 3.0055 & PROMPT-3 & $B$ & 3520.0 & $20.85 \pm 0.24$ \\
\hline 2009 Sep 29.17 & 3.0056 & PROMPT-4 & $R$ & 2960.0 & $19.96 \pm 0.09$ \\
\hline 2009 Sep 29.17 & 3.0083 & PROMPT-2 & $V$ & 3600.0 & $20.64 \pm 0.43$ \\
\hline 2009 Sep 29.53 & 3.3664 & UVOT & $V$ & 2533.9 & $20.75 \pm 0.13$ \\
\hline 2009 Sep 29.54 & 3.3796 & UVOT & $U$ & 3233.8 & $20.58 \pm 0.07$ \\
\hline 2009 Sep 30.00 & 3.8439 & PROMPT-5 & $I$ & 5840.0 & $19.89 \pm 0.11$ \\
\hline 2009 Sep 30.00 & 3.8446 & PROMPT-4 & $R$ & 6560.0 & $20.39 \pm 0.10$ \\
\hline 2009 Sep 30.08 & 3.9295 & PROMPT-5 & $I$ & 6000.0 & $19.95 \pm 0.12$ \\
\hline 2009 Sep 30.08 & 3.9302 & PROMPT-4 & $R$ & 5680.0 & $20.65 \pm 0.14$ \\
\hline 2009 Sep 30.17 & 4.0116 & PROMPT-3 & $B$ & 30640.0 & $21.29 \pm 0.13$ \\
\hline 2009 Sep 30.17 & 4.0121 & PROMPT-2 & $V$ & 28720.0 & $20.71 \pm 0.17$ \\
\hline 2009 Sep 30.17 & 4.0249 & PROMPT-5 & $I$ & 7440.0 & $20.10 \pm 0.12$ \\
\hline 2009 Sep 30.17 & 4.0253 & PROMPT-4 & $R$ & 7520.0 & $20.55 \pm 0.09$ \\
\hline 2009 Sep 30.29 & 4.1428 & PROMPT-5 & $I$ & 10400.0 & $20.18 \pm 0.11$ \\
\hline 2009 Sep 30.29 & 4.1445 & PROMPT-4 & $R$ & 10400.0 & $20.73 \pm 0.10$ \\
\hline 2009 Sep 30.55 & 4.3878 & UVOT & $V$ & 3091.7 & $20.60 \pm 0.11$ \\
\hline 2009 Sep 30.55 & 4.3944 & UVOT & $U$ & 3341.7 & $20.90 \pm 0.08$ \\
\hline 2009 Oct 1.04 & 4.8842 & PROMPT-4 & $R$ & 12640.0 & $20.73 \pm 0.10$ \\
\hline 2009 Oct 1.04 & 4.8854 & PROMPT-5 & $I$ & 12400.0 & $20.17 \pm 0.10$ \\
\hline 2009 Oct 1.11 & 4.9658 & SMARTS/ANDICAM & $I$ & 2160.0 & $20.33 \pm 0.10$ \\
\hline 2009 Oct 1.11 & 4.9658 & SMARTS/ANDICAM & $J$ & 1800.0 & $19.16 \pm 0.13$ \\
\hline 2009 Oct 1.13 & 4.9841 & PROMPT-2 & $V$ & 23520.0 & $21.15 \pm 0.31$ \\
\hline 2009 Oct 1.17 & 4.9896 & PROMPT-3 & $B$ & 24240.0 & $21.32 \pm 0.17$ \\
\hline 2009 Oct 1.25 & 5.1003 & PROMPT-5 & $I$ & 12640.0 & $20.17 \pm 0.11$ \\
\hline 2009 Oct 1.25 & 5.1024 & PROMPT-4 & $R$ & 11920.0 & $20.92 \pm 0.13$ \\
\hline 2009 Oct 1.64 & 5.4915 & UVOT & $U$ & 4142.8 & $21.36 \pm 0.10$ \\
\hline 2009 Oct 2.04 & 5.8878 & PROMPT-4 & $R$ & 11600.0 & $20.83 \pm 0.11$ \\
\hline 2009 Oct 2.17 & 5.9904 & PROMPT-2 & $V$ & 21360.0 & $21.14 \pm 0.33$ \\
\hline 2009 Oct 2.17 & 6.0045 & PROMPT-3 & $B$ & 28400.0 & $21.71 \pm 0.25$ \\
\hline 2009 Oct 2.25 & 6.0812 & PROMPT-4 & $R$ & 16960.0 & $21.04 \pm 0.11$ \\
\hline 2009 Oct 2.25 & 6.0880 & PROMPT-5 & $I$ & 15040.0 & $20.61 \pm 0.15$ \\
\hline 2009 Oct 3.00 & 6.8605 & PROMPT-4 & $R$ & 4240.0 & $>21.34$ \\
\hline 2009 Oct 3.04 & 6.8617 & PROMPT-5 & $I$ & 4000.0 & $>20.65$ \\
\hline 2009 Oct 3.04 & 6.8650 & PROMPT-3 & $B$ & 3520.0 & $>21.00$ \\
\hline 2009 Oct 3.00 & 6.8781 & UVOT & $U$ & 8789.3 & $21.59 \pm 0.08$ \\
\hline 2009 Sep 4.74 & 8.5887 & UVOT & $U$ & 4052.7 & $22.09 \pm 0.18$ \\
\hline 2009 Oct 5.17 & 8.9975 & PROMPT-4 & $R$ & 12400.0 & $21.36 \pm 0.26$ \\
\hline 2009 Oct 5.17 & 9.0014 & PROMPT-5 & $I$ & 13280.0 & $>21.35$ \\
\hline 2009 Oct 5.49 & 9.3449 & UVOT & $U$ & 4640.7 & $22.07 \pm 0.16$ \\
\hline 2009 Oct 6.38 & 10.2257 & UVOT & $U$ & 4392.5 & $22.84 \pm 0.29$ \\
\hline 2009 Oct 7.17 & 11.0096 & PROMPT-4 & $R$ & 27840.0 & $22.09 \pm 0.22$ \\
\hline 2009 Oct 7.21 & 11.0377 & PROMPT-5 & $I$ & 23680.0 & $>21.47$ \\
\hline 2009 Oct 7.42 & 11.2777 & UVOT & $U$ & 5096.2 & $22.22 \pm 0.17$ \\
\hline 2009 Oct 8.32 & 12.1742 & UVOT & $U$ & 4573.2 & $22.94 \pm 0.33$ \\
\hline 2009 Oct 19.05 & 22.90 & Gemini-S/GMOS & $g^{\prime}$ & 600.0 & $24.56 \pm 0.11$ \\
\hline 2009 Oct 19.05 & 22.90 & Gemini-S/GMOS & $r^{\prime}$ & 600.0 & $23.97 \pm 0.11$ \\
\hline 2009 Oct 19.05 & 22.90 & Gemini-S/GMOS & $i^{\prime}$ & 600.0 & $23.67 \pm 0.12$ \\
\hline
\end{tabular}

Notes.

${ }^{a}$ UT at the beginning of exposure.

b Time from midpoint of exposure to Fermi-GBM trigger.

${ }^{\mathrm{c}}$ Reported magnitudes have not been corrected for Galactic extinction $\left(E(B-V)=0.024 \mathrm{mag}\right.$; Schlegel et al. 1998). Observations in the $g^{\prime}$,

$r^{\prime}$, and $i^{\prime}$ bands are reported on the AB magnitude system (Oke \& Gunn 1983). All other filters are referenced to Vega.

Finally, the field of GRB 090926A was observed in the radio $(5.5 \mathrm{GHz})$ on 2009 October 1 with the Australia Telescope Compact Array. No source was detected at the afterglow location to a $2 \sigma$ limit of $f_{v}<1.5 \mathrm{mJy}$ (Moin et al. 2009).

\subsubsection{Spectroscopy}

Malesani et al. (2009) obtained a spectrum of the afterglow of GRB 090926A with the X-Shooter instrument mounted on the
$8 \mathrm{~m}$ Very Large Telescope (VLT) UT2. Based on the detection of a damped-Ly $\alpha$ system and many strong, narrow absorption features, these authors derive a redshift of 2.1062 for the host galaxy of GRB 090926A. Rau et al. (2010) find a consistent result based on a spectrum taken with FORS2 on the VLT.

At this redshift, the prompt isotropic gamma-ray energy release in the $1-10^{4} \mathrm{keV}$ bandpass is $E_{\gamma \text {,iso }}=(1.89 \pm 0.03) \times$ $10^{54} \mathrm{erg}$. Assuming a nonthermal spectrum up to $E_{\mathrm{obs}}=20 \mathrm{GeV}$, 


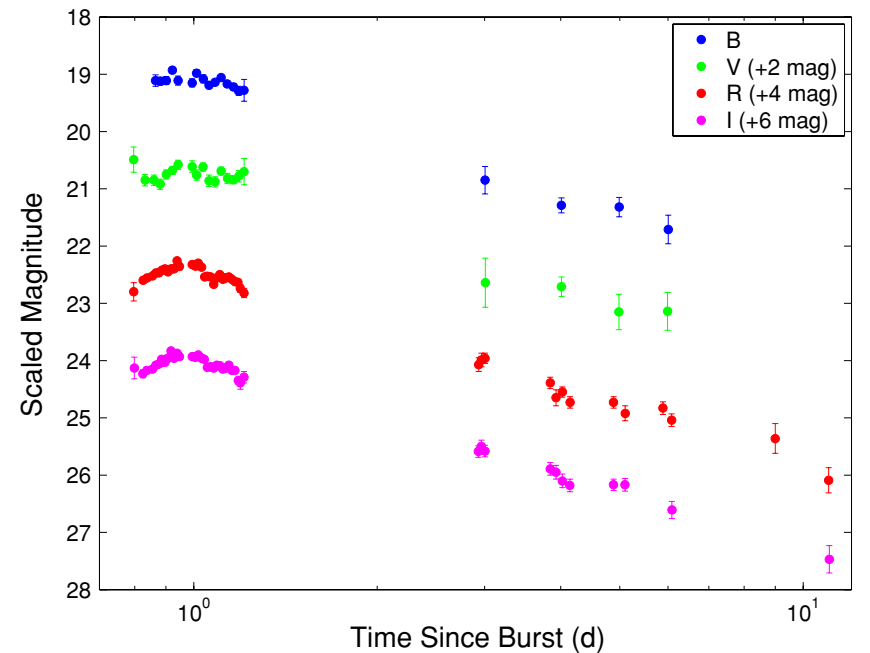

Figure 8. PROMPT $B V R I$ observations of GRB 090926A. The afterglow experiences a prominent rebrightening in all filters, peaking at $t \approx 1 \mathrm{day}$. Such behavior is difficult to reconcile with standard afterglow theory, and may suggest a late-time $\left(t \gg \Delta t_{\mathrm{GRB}}\right)$ injection of energy from the central engine (Rees \& Meszaros 1998).

(A color version of this figure is available in the online journal.)

we infer a lower limit for the initial Lorentz factor of $\Gamma_{0} \gtrsim 700$ (Lithwick \& Sari 2001).

\section{AFTERGLOW MODELING AND RESULTS}

In the standard "fireball” model of GRBs (e.g., Piran 2005), a compact central engine (either a black hole or a rapidly spinning proto-neutron star) drives a collimated $\left(\theta \lesssim 10^{\circ}\right)$, ultrarelativistic $\left(\Gamma_{0} \gtrsim 100\right)$ outflow of matter and/or radiation. Some dissipative process within the outflow (possibly collisionless shocks) gives rise to the prompt gamma-ray emission, converting some fraction $\eta_{\gamma} \equiv E_{\gamma \text {,iso }} /\left(E_{\gamma \text {,iso }}+E_{\mathrm{KE} \text {,iso }}\right)$ of the total relativistic energy to high-energy radiation.

The outflow is ultimately slowed as it sweeps up and shock heats the circumburst medium, and synchrotron radiation from electrons accelerated at the shock front causes the broadband afterglow. The resulting spectrum is well described as a series of broken power laws with three characteristic frequencies: $v_{a}$, the frequency below which the radiation is self-absorbed; $v_{m}$, the characteristic frequency of the emitting electrons; and $v_{c}$, the frequency above which electrons are able to cool efficiently through radiation (e.g., Granot \& Sari 2002).

The temporal evolution of the afterglow depends on the density profile of the circumburst medium. We consider here two possibilities: a constant-density circumburst medium $[\rho(r) \propto$ $\left.r^{0}\right]$, as would be expected in an environment similar to the interstellar medium (ISM; Sari et al. 1998), and a wind-like environment $\left[\rho(r) \propto r^{-2}\right]$, as would be the case for a massivestar progenitor that sheds its outer envelope at a constant rate (Chevalier \& Li 2000).

At early times, the afterglow emission appears isotropic to distant observers due to the effects of relativistic beaming. However, the outflow slows as it sweeps up more and more circumburst material. When $\Gamma(t) \approx 1 / \theta$, lateral spreading of the jet becomes important and observers will notice "missing" emission from wide angles (Rhoads 1999; Sari et al. 1999). This hydrodynamic transition manifests itself as an achromatic steepening in the afterglow light curve. Measuring the time of this jet break $\left(t_{\mathrm{j}}\right)$ allows us to infer the opening angle of the outflow.
In order to ascertain the total relativistic energy output from a GRB, we require three measurements: (1) $E_{\gamma, \text { iso }}$, the isotropic energy release in the prompt gamma-ray emission, which is inferred from the high-energy fluence and the associated afterglow or host redshift; (2) $\theta$, the half-opening angle of the beamed emission, inferred from the detection of a jet break; and (3) $E_{\mathrm{KE}}$, the kinetic energy of the blast wave that is powering the broadband afterglow, which can be inferred either via afterglow modeling or, more accurately, from late-time radio calorimetry in the nonrelativistic phase (Berger et al. 2004; Frail et al. 2005; van der Horst et al. 2008). We stress here that we are neglecting contributions from nonelectromagnetic phenomena (neutrinos, gravity waves, etc.) and slower moving material (i.e., $\mathrm{SN}$ emission), and so are providing only lower limits on the total energy budget.

Unfortunately, none of the radio afterglows in our sample of LAT events is sufficiently bright to perform calorimetry in the nonrelativistic phase. Instead we take the approach of applying the standard relativistic blast-wave model to these multi-wavelength afterglow data. Our objective is to translate the three critical frequencies observed, together with the peak flux density, $F_{v, \max }$, and the jet break time, $t_{\mathrm{j}}$, into a physical description of the outflow. In particular, we shall attempt to estimate seven parameters: $E_{\mathrm{KE}}$, the kinetic energy of the blast wave; $n$, the density of the circumburst medium (or, alternatively for a wind-like circumburst medium, $A_{*}$, where $\left.\rho=5 \times 10^{11} A_{*} r^{-2}\right)^{26} ; \epsilon_{e}$, the fraction of the total energy apportioned to electrons; $\epsilon_{B}$, the fraction of the total energy apportioned to the magnetic field; $p$, the electron power-law index; $A_{V}$, the host-galaxy extinction ${ }^{27}$; and $\theta$, the jet opening angle.

The particular method that we use has been described in some detail in the literature (Harrison et al. 2001; Yost et al. 2002; Frail et al. 2003; Chandra et al. 2008; Cenko et al. 2010) and a similar approach has been taken by Panaitescu \& Kumar (2000, 2001a, 2001b). In brief, we assume a spherical or jet outflow expanding relativistically into either a constant-density medium or one with a radial $r^{-2}$ profile, caused by the mass-loss rate from a progenitor star. The dynamics and hence the evolution of the break frequencies is governed by the Blandford-McKee solution. We adopt a simple broken-power-law evolution for the jet, using the time dependences from Sari et al. (1999). Our afterglow emission is dominated by synchrotron emission, but we include both corrections for radiative losses and inverseCompton emission (Sari \& Esin 2001).

To account for differences in instrumental configurations, we have applied a $7 \%$ cross-calibration uncertainty to all data points before calculating the models. All reported uncertainties have been determined using a Markov-Chain Monte Carlo bootstrap analysis with 1000 trials and represent only statistical errors associated with the fit. Systematic errors associated with model uncertainties are likely much larger and difficult to estimate. Both Yost et al. (2003) and Panaitescu \& Kumar (2002) have explored the sensitivity of solutions on various model assumptions in an effort to quantify the systematics

\footnotetext{
26 For a wind-like medium, the circumburst density is normalized for a progenitor mass-loss rate of $\dot{M}=10^{-5} M_{\odot} \mathrm{yr}^{-1}$ and a wind speed of $v_{w}=1000 \mathrm{~km} \mathrm{~s}^{-1}$ (Chevalier \& Li 1999). The units of $A_{*}$ are thus $\mathrm{g} \mathrm{cm}^{-1}$, and the conversion to a particle density (as a function of radius) is given by $n=30 A_{*} r_{17}^{-2} \mathrm{~cm}^{-3}$.

27 We have assumed an SMC-like extinction curve for all events here (Pei 1992; Kann et al. 2006). Given the relatively modest amounts of dust inferred for these host galaxies, this choice does not affect any of our primary conclusions.
} 
Table 8

GRB 090323 Afterglow Fit Parameters

\begin{tabular}{|c|c|c|c|c|c|c|c|c|}
\hline Model & $\begin{array}{c}E_{\mathrm{KE}, \text { iso }} \\
\left(10^{52} \mathrm{erg}\right)\end{array}$ & $\begin{array}{c}A_{*} \\
\left(\mathrm{~g} \mathrm{~cm}^{-1}\right)\end{array}$ & $\begin{array}{c}\epsilon_{e} \\
(\%)\end{array}$ & $\begin{array}{l}\epsilon_{B} \\
(\%)\end{array}$ & $\begin{array}{c}\theta \\
\left({ }^{\circ}\right)\end{array}$ & $p$ & $\begin{array}{c}A_{V} \text { (host) } \\
(\mathrm{mag})\end{array}$ & $\chi_{v}^{2}(\mathrm{dof})$ \\
\hline Wind & $116_{-9}^{+13}$ & $0.10 \pm 0.01$ & $7.0 \pm 0.5$ & $0.89_{-0.18}^{+0.07}$ & $2.8_{-0.1}^{+0.4}$ & $2.71 \pm 0.02$ & $0.12 \pm 0.07$ & $1.33(239)$ \\
\hline Wind-Iso $^{\mathrm{a}}$ & $\ldots$ & .. & $\ldots$ & ${ }^{-0.18}$ & 90 & $\ldots$ & $\ldots$ & $1.35(240)$ \\
\hline ISM & 623 & 0.11 & $33^{\mathrm{b}}$ & 0.13 & 2.8 & 2.10 & 0.19 & 2.45 (239) \\
\hline
\end{tabular}

Notes.

${ }^{a}$ For the isotropic model, all parameters are identical to the jetted model, except for the opening angle, which is held fixed.

$\mathrm{b}$ The electron partition fraction was fixed to its equipartition value.

and limitations of these fitting methods. Recently, more GRB afterglow emission has been calculated using detailed numerical simulations (e.g., Zhang \& MacFadyen 2009; van Eerten et al. 2010,2011 ), but with few exceptions the quality of the afterglow data is not sufficient to make detailed comparisons of methods. We return to the issue of systematic uncertainties in our energy measurements in Section 4.4.

\subsection{GRB 090323}

\subsubsection{Preliminary Considerations}

Before proceeding to a detailed model, we derive some initial constraints by looking at the spectral and temporal behavior of the afterglow. This type of " $\alpha-\beta$ " analysis is useful for comparing to other published work, but we do not use it in our detailed afterglow modeling.

Considering first the X-ray afterglow, we fit the flux density to a power-law decay and find a best-fit index of $\alpha_{\mathrm{X}}=1.5 \pm 0.1$ $\left(\chi^{2}=28.2\right.$ for 22 degrees of freedom (dof)). Performing an analogous power-law fit to the X-ray spectrum, we find $\beta_{\mathrm{X}}=1.06_{-0.13}^{+0.34}\left(\chi^{2}=8.0\right.$ for 13 dof; see the online compilation of N.R.B. for details).

We perform a similar power-law fit in the optical/NIR $\left(f_{\nu} \propto t^{-\alpha} v^{-\beta}\right)$, forcing the decay index to be identical in all filters and ignoring the points at $t>10$ days due to hostgalaxy contamination (see below). Though the fit quality is somewhat worse $\left(\chi^{2}=153.3\right.$ for 99 dof $)$, we find similar power-law indices as seen in the X-rays: $\alpha_{\mathrm{O}}=1.74 \pm 0.05$; $\beta_{\mathrm{O}}=1.14 \pm 0.06$.

For typical values of the electron index $p \approx 2.5$ (Shen et al. 2006; Starling et al. 2008; Curran et al. 2009), this steep temporal decay is difficult to reconcile with expansion into a constantdensity medium, where $\alpha$ is typically $\sim 1.0-1.5$ (Sari et al. 1998). However, expansion into a wind-like medium, even with relatively standard electron indices, can naturally accommodate such a temporal decay (Chevalier \& Li 2000).

The temporal decay index we derive is slightly shallower than that inferred by McBreen et al. $\left(2010 ; \alpha_{O}=1.90\right)$. These authors include an additional emission mechanism beyond the standard synchrotron emission, a "bump" at $t \approx 7$ days that effectively removes flux from the power-law component at late times. Our derived spectral index is noticeably shallower than that found by McBreen et al. $\left(2010 ; \beta_{\mathrm{O}}=0.65\right)$, predominantly because we have yet to include the effects of dust extinction in the GRB host galaxy. We shall correct for this in our full modeling in the following section.

The comparable spectral and temporal indices in the optical and X-rays suggest that both bandpasses fall in the same synchrotron spectral regime. In other words, the cooling frequency should fall either below the optical or above the X-ray bandpass for the duration of these observations ( $t \approx 1-10$ days).
Examining the "closure" relations between $\alpha$ and $\beta$ in different circumburst media and spectral regimes (e.g., Price et al. 2002), we find that we can rule out a low cooling frequency at large significance, as this would require $\alpha=(3 \beta-1) / 2 \approx 1.0$ for both a wind-like and an ISM-like circumburst medium.

The relatively flat radio light curve (modulo effects from ISS) until $t \approx 20$ days has two important implications. First, it suggests a wind-like circumburst medium, as the flux would be expected to rise in proportion to $t^{1 / 2}$ for $v_{a}<v<v_{m}$ in a constant-density environment. Because of the effects of ISS, however, the statistical significance is not very large and the full suite of broadband observations is required to compare the competing circumburst density profiles. Second, together with the lack of evidence for late-time steepening in the $\mathrm{X}$-ray and optical light curves, this suggests that if a jet break is required, it should occur at $t \gtrsim 20$ days. (Another possibility for the decay could be the peak frequency $v_{m}$ passing through the radio bands.)

Finally, the relatively bright $r^{\prime}$-band detection at $t=130$ days is almost certainly dominated by flux from the host galaxy and not the afterglow of GRB 090323. If we assume this flux is due entirely to host light, the host will contribute a significant fraction of the flux in some of the late-time points of our optical light curve ( $\sim 40 \%$ at $t=14$ days in $\left.r^{\prime}\right)$. A similar conclusion is reached by $\mathrm{McBreen}$ et al. (2010) who also detect the host in the $i^{\prime}$ filter. In our detailed modeling, we assume a constant contribution from the host galaxy equal to the values from these measurements in the $R / r^{\prime}$ and $i^{\prime}$ filters.

\subsubsection{Modeling Results}

With these constraints in hand, we have modeled the afterglow of GRB 090323 with the software described above. The resulting best-fit wind model is plotted in Figure 2 and the derived parameters are provided in Table 8 . The overall fit quality is reasonable $\left(\chi^{2}=316.8\right.$ for 239 dof $)$, with comparable residuals in the radio, optical, and X-ray bandpasses. The best-fit ISM model results in a dramatically worse fit $\left(\chi^{2}=585.0\right.$ for 239 dof), with poorer residuals in all bandpasses (i.e., not just the radio).

As expected, the cooling frequency $v_{c}$ lies near or above the $\mathrm{X}$-ray bandpass for the duration of our observations ( $t \gtrsim 1$ day). Consequently, the ratio of $E_{\mathrm{KE} \text {,iso }}$ to $A_{*}$ is required to be larger than inferred for previous GRBs (e.g., Panaitescu \& Kumar 2001b; Yost et al. 2003). While the isotropic blast-wave kinetic energy is relatively large compared to that of previously modeled GRBs $\left(E_{\mathrm{KE} \text {, iso }}=1.2 \times 10^{54} \mathrm{erg}\right)$, it is in fact comparable to the prompt gamma-ray energy release, as would be expected for reasonable values of the gamma-ray efficiency $\eta_{\gamma}$. The inferred density is slightly lower than usual $\left(A_{*}=0.10 \mathrm{~g} \mathrm{~cm}^{-1}\right)$, though smaller values have been reported in the literature (e.g., GRB 020405; Chevalier et al. 2004). 
Table 9

GRB 090328 Afterglow Fit Parameters

\begin{tabular}{|c|c|c|c|c|c|c|c|c|}
\hline Model & $\begin{array}{c}E_{\mathrm{KE}, \text { iso }} \\
\left(10^{52} \mathrm{erg}\right)\end{array}$ & $\begin{array}{c}A_{*} \\
\left(\mathrm{~g} \mathrm{~cm}^{-1}\right)\end{array}$ & $\begin{array}{c}\epsilon_{e} \\
(\%)\end{array}$ & $\begin{array}{l}\epsilon_{B} \\
(\%)\end{array}$ & $\begin{array}{c}\theta \\
\left(^{\circ}\right)\end{array}$ & $p$ & $\begin{array}{c}A_{V} \text { (host) } \\
\text { (mag) }\end{array}$ & $\chi_{v}^{2}(\mathrm{dof})$ \\
\hline Wind & $82_{-18}^{+28}$ & $0.26_{-0.07}^{+0.13}$ & $11_{-1}^{+6}$ & $0.19_{-0.08}^{+0.04}$ & $4.2_{-0.8}^{+1.3}$ & $2.81_{-0.07}^{+0.14}$ & $0.33 \pm 0.05$ & $0.97(101)$ \\
\hline Wind-Iso $^{\mathrm{a}}$ & $\ldots$ & $\ldots$ & $\ldots$ & $\ldots$ & 90 & $\ldots$ & $\ldots$ & $1.63(102)$ \\
\hline ISM & 87 & 82 & 19 & 0.12 & 7.6 & 2.26 & 0.30 & $1.11(101)$ \\
\hline
\end{tabular}

Note. ${ }^{\text {a }}$ For the isotropic model, all parameters are identical to the jetted model, except for the opening angle, which is held fixed.

The best-fit model results in a jet break time of $t_{\mathrm{j}}=$ $17.6_{-2.3}^{+11.2}$ days. This occurs largely after the X-ray and optical observations have ceased (except for the host detections in the optical), and is therefore most directly constrained by the radio light curve. In Figure 2, we also plot the same model parameters but for an isotropic explosion, where the turnover in the radio at late times is due to the peak frequency $v_{m}$ passing through the radio bandpass (see also Table 8). For the entire data set, the fit quality for the isotropic model is only marginally worse $\left(\chi^{2}=324.3\right.$ for 240 dof $)$; however, this masks the significant improvement in the fit achieved in the radio bandpass $\left(\chi^{2}=7.8\right.$ for the jet-like model compared to $\chi^{2}=13.5$ for the isotropic model). Conservatively, the opening angle and beaming-corrected energetics derived here can at worst be treated as lower limits.

Though the upper bound on the jet break time is not extremely well constrained, due to the relatively weak dependence of $\theta$ on $t_{\mathrm{j}}$ in a wind-like circumburst medium (Equation (5)), the uncertainty in the jet opening angle is significantly smaller. Compared with previous GRB samples, the jet break occurs rather late in the observer frame (e.g., Zeh et al. 2006), and the inferred opening angle $(\theta=2.8)$ is still relatively small compared with previous samples. Though the dependence is not strong $\left(\theta \propto\left(E_{\mathrm{KE}, \text { iso }} / A_{*}\right)^{-1 / 4}\right)$, the large ratio of $E_{\mathrm{KE} \text {,iso }}$ to $A_{*}$ effectively lowers the opening angle for a given jet break time.

After applying the collimation correction, we find that the true energy release of GRB 090323 is $E_{\gamma}=4.0_{-0.4}^{+1.2} \times 10^{51} \mathrm{erg}$, $E_{\mathrm{KE}}=1.4_{-0.2}^{+0.6} \times 10^{51} \mathrm{erg}$. We compare these results with a larger sample of events in Section 4.1.

\subsection{GRB 090328}

\subsubsection{Preliminary Considerations}

Following the results of Section 3.1.1, we first consider the spectral and temporal indices of GRB 090328 in the observed bandpasses independently. The X-ray afterglow light curve is well fit by a single power-law decay with index $\alpha_{\mathrm{X}}=1.65 \pm 0.09$ $\left(\chi^{2}=24.1\right.$ for 26 dof $)$. The spectral index, on the other hand, is less well constrained: $\beta_{\mathrm{X}}=1.1_{-0.3}^{+0.4}\left(\chi^{2}=6.7\right.$ for 14 dof). We note that the X-ray spectral fit indicates the presence of an additional absorption component in the host galaxy of $\operatorname{GRB} 090328\left(N_{\mathrm{H}}=4.9_{-2.8}^{+3.5} \times 10^{21} \mathrm{~cm}^{-2}\right.$ at $\left.z=0.736\right)$.

Along the lines of our analysis of the optical light curve in Section 3.1.1, we fit our Swift $U$-band observations, together with the optical/NIR data from GROND (McBreen et al. 2010), to a power-law model of the form $f_{v} \propto t^{-\alpha} v^{-\beta}$. After subtracting the host flux values derived in each filter from GROND photometry, we find a relatively poor fit $\left(\chi^{2}=99.7\right.$ for $41 \mathrm{dof}$ ) with $\alpha_{\mathrm{O}}=1.85 \pm 0.13$ and $\beta_{\mathrm{O}}=1.40 \pm 0.05$. The resulting spectral index is comparable to that found by McBreen et al. (2010; before we account for host reddening; see below), though again we derive a somewhat shallower temporal index due to the fact that these authors added a bump to their lightcurve model at late times.

In the case of GRB 090328, examining the $\alpha-\beta$ closure relations yields little insight. Because of the poor fit quality in the optical and the large uncertainty in the X-ray spectral index, we are unable to rule out much of the available parameter space. We note, however, that with the exception of the excess absorption, the X-ray and optical afterglow light curves and spectral energy distributions of GRB 090328 broadly resemble those of GRB 090323.

\subsubsection{Modeling Results}

The results of our best-fit model for the afterglow of GRB 090328 are shown in Figure 4 and the parameters are provided in Table 9 . The overall fit quality is quite good $\left(\chi^{2}=98.4\right.$ for $101 \mathrm{dof}$ ). The improvement over our simple power-law fits in the optical in the previous section results largely from incorporating the modest host-galaxy extinction $\left(A_{V}=0.33 \mathrm{mag}\right)$. Only the early radio light curve, which suffers from strong scintillation at early times, differs systematically from the model predictions.

Much like the case of GRB 090323, we find that only a wind-like circumburst medium is favored, though not nearly in as statistically significant a manner. The largest discrepancy between the wind-like and constant-density environments actually occurs in the X-ray bandpass, where the wind is strongly favored $\left(\chi_{\nu, \text { wind }}^{2}=1.08\right.$ compared with $\left.\chi_{\nu, \text { ISM }}^{2}=1.48\right)$.

The steep decline in the radio light curve at late times offers relatively tight constraints on the presence of a jet break. The difference in model fits for the radio band is $\chi_{\nu}^{2}=2.52$ (beamed) compared with $\chi_{v}^{2}=10.81$ (isotropic), a fact clearly evidenced by the rising isotropic light curve in Figure 4. In fact, the break is consistent with (though not formally required by) the final few points in the X-ray light curve as well. For our best-fit model, we infer $t_{\mathrm{j}}=9.0_{-6.0}^{+11.6}$ days and $\theta=\left(4.2_{-0.8}^{+1.3}\right)^{\circ}$. The break is essentially impossible to detect in the optical due to the presence of the bright host galaxy.

Correcting for the effects of beaming, we find an energy release of $E_{\gamma}=2.6_{-1.1}^{+2.3} \times 10^{50} \mathrm{erg}$ and $E_{\mathrm{KE}}=2.2_{-0.9}^{+2.9} \times 10^{51} \mathrm{erg}$. The total relativistic energy release, $E_{\text {tot }} \approx 2 \times 10^{51} \mathrm{erg}$, is somewhat less than the value we derive for GRB 090323 . We note that unlike most of the other events in our sample, the implied gamma-ray efficiency for GRB 090328 is relatively modest.

\subsection{GRB 090902B}

\subsubsection{Preliminary Considerations}

A single power-law model with $\alpha_{\mathrm{X}}=1.36 \pm 0.03$ provides a good fit to the X-ray light curve of GRB 090902B over 
Table 10

GRB 090902B Afterglow Fit Parameters

\begin{tabular}{|c|c|c|c|c|c|c|c|c|}
\hline Model & $\begin{array}{c}E_{\mathrm{KE}, \text { iso }} \\
\left(10^{52} \mathrm{erg}\right)\end{array}$ & $\begin{array}{c}n \\
\left(\mathrm{~cm}^{-3}\right)\end{array}$ & $\begin{array}{c}\epsilon_{e} \\
(\%)\end{array}$ & $\begin{array}{c}\epsilon_{B} \\
(\%)\end{array}$ & $\begin{array}{c}\theta \\
\left({ }^{\circ}\right)\end{array}$ & $p$ & $\begin{array}{c}A_{V} \text { (host) } \\
\text { (mag) }\end{array}$ & $\chi_{v}^{2}($ dof $)$ \\
\hline ISM & $56_{-3}^{+7}$ & $\left(5.6_{-0.7}^{+0.9}\right) \times 10^{-4}$ & $13 \pm 1$ & $33^{\mathrm{a}}$ & $3.9 \pm 0.2$ & $2.21 \pm 0.02$ & $0.05 \pm 0.03$ & $1.11(103)$ \\
\hline ISM-Iso $^{b}$ & $\cdots$ & -0 & $\ldots$ & $\cdots$ & 90 & $\ldots$ & $\ldots$ & $1.79(104)$ \\
\hline Wind & 0.063 & 0.19 & 8.9 & $33^{\mathrm{a}}$ & 28 & 1.54 & 0.04 & $1.54(103)$ \\
\hline
\end{tabular}

Notes.

a The electron partition fraction was fixed to its equipartition value.

${ }^{\mathrm{b}}$ For the isotropic model, all parameters are identical to the jetted model, except for the opening angle, which is held fixed.

the entire span of the XRT observations $\left(\chi^{2}=94.8\right.$ for 107 dof). Likewise, the X-ray spectrum is well fit by a power law with $\beta_{\mathrm{X}}=0.90 \pm 0.13\left(\chi^{2}=42.8\right.$ for 56 dof $)$. The X-ray spectrum strongly favors the presence of absorption in excess of the Galactic value along the GRB line of sight. Assuming the dust arises in the GRB host galaxy at $z=1.82$, the best-fit host column density is $N_{\mathrm{H}}=\left(6.3_{-1.7}^{+2.1}\right) \times 10^{21} \mathrm{~cm}^{-2}$. These results are all consistent with a similar analysis of the X-ray afterglow performed by Pandey et al. (2010).

The decline inferred from the early-time ROTSE-III $R$-band observations is significantly steeper than the late-time ( $t \gtrsim 1$ day) optical decay. Following Pandey et al. (2010), we attribute this emission largely to the presence of a reverse shock and do not include these data points in any subsequent analysis. Simultaneously fitting the remaining optical data to a power law of the form $f_{v} \propto t^{-\alpha} v^{-\beta}$, we find $\alpha_{\mathrm{O}}=0.89 \pm 0.03$ and $\beta_{\mathrm{O}}=0.82 \pm 0.10\left(\chi^{2}=113.1\right.$ for $\left.67 \mathrm{dof}\right)$. Again, our results are consistent with those reported by Pandey et al. (2010) and McBreen et al. (2010).

The large discrepancy between the X-ray and optical temporal decay slopes suggests that the two bandpasses fall in different synchrotron spectral regimes (i.e., $v_{\mathrm{O}}<v_{c}<v_{\mathrm{X}}$ ). Furthermore, the relatively shallow optical decline strongly rules out a windlike circumburst medium. We find that both the X-ray and optical bandpasses are broadly consistent with expansion into a constant-density medium and an electron index $p \approx 2.2$. The observed X-ray temporal decline is somewhat steeper than what is predicted, but this could be accounted for by synchrotron radiative losses at later times.

The radio light curve appears to decay from the earliest observations at $t_{0}+1.5$ days onward. This behavior is not expected in the simple forward-shock model where radio afterglows are typically observed to rise (for a constant-density medium) or remain flat (for a stratified environment) until either a jet break occurs or the peak frequency reaches the radio bandpass (typically weeks after the burst). While ISS can affect the radio afterglow dramatically, particularly at early times, such behavior nonetheless requires an alternate explanation.

Bright, early-time radio emission is actually rather common, having been detected in a quarter of all radio afterglows to date (Soderberg \& Ramirez-Ruiz 2003; P. Chandra et al. 2011, in preparation). This prompt component is most commonly attributed to the reverse shock propagating in the shocked ejecta. The reverse-shock emission fades away quite quickly, and at late times ( $t \gtrsim 5$ days) the afterglow will be dominated by the forward-shock emission. GRB 990123 was the first known example (Kulkarni et al. 1999), but there have been many more claimed detections since (e.g., Berger et al. 2003b; Panaitescu 2005; Chandra et al. 2010).

The radio light curve of GRB 090902B most closely resembles that of GRB 991216 in which the early power-law decline is due to the overlap of emission from the reverse and forward shocks (Frail et al. 2000b). Since we are concerned here with the modeling of the behavior of the forward shock, we will exclude the early radio point and postpone a full discussion to a subsequent paper dealing with a compilation of all known GRBs having prompt radio emission (P. Chandra et al. 2011, in preparation).

\subsubsection{Modeling Results}

Using the constraints derived above, our best-fit model for GRB 090902B assuming a constant-density circumburst medium is shown in Figure 6, and the derived model parameters are presented in Table 10. The overall fit quality is quite reasonable $\left(\chi^{2}=114.8\right.$ for 102 dof $)$, with comparable fit quality in all bandpasses.

We stress, however, that we have not included the first two $R$-band data points and the first VLA detection in our fitting, as we believe the flux at these points is dominated by reverseshock emission. These points are severely underpredicted by our models and, if included, could result in somewhat different fit parameters. Including the early-time radio emission at $t \approx$ 1.5 days would not dramatically affect our results, however, due mostly to the large effects of ISS.

We find that a wind-like environment is strongly disfavored by our models, with the best-fit wind model having $\chi^{2}=$ 156.7 (102 dof). Furthermore, the parameters of the best-fit wind model are relatively unphysical, with an extremely low afterglow energy $\left(E_{\mathrm{KE} \text {,iso }}=6.3 \times 10^{50} \mathrm{erg}\right.$, more than four orders of magnitude less than the prompt gamma-ray energy) and an electron energy distribution index of $p=1.54$ ( $p$ values less than 2 require a spectral cutoff to avoid an infinite energy).

Much like the other events considered here, our inferred parameters for GRB 090902B suggest a large afterglow kinetic energy $\left(E_{\mathrm{KE} \text {,iso }}=5.6_{-0.7}^{+0.3} \times 10^{53} \mathrm{erg}\right.$; a factor of five less than the prompt gamma-ray energy release) and a low circumburst density $\left(n=5.6_{-0.7}^{+0.9} \times 10^{-4} \mathrm{~cm}^{-3}\right)$. In fact, we are unable to find any acceptable solutions $\left(\chi_{v}^{2}<2\right)$ with $n>10^{-2} \mathrm{~cm}^{-3}$. The inferred circumburst density for many long-duration GRBs is lower than typical values observed in dense molecular clouds (where presumably their massive-star progenitors have formed). Yet the value we have derived for GRB 090902B is closer in fact to what one might find in the ambient ISM or even the intergalactic medium, as has been found for the few shorthard GRBs with sufficient afterglow data (e.g., Panaitescu 2006; Perley et al. 2009b). We return to this issue in more detail in Section 4.3.

Isotropic models are strongly disfavored as well $\left(\chi^{2}=182.8\right.$ for 104 dof), with evidence for a jet break from both the optical (in particular the late-time measurement from McBreen et al. 2010 at $t \approx 23$ days) and the radio. Our models suggest that 
Table 11

GRB 090926A Afterglow Fit Parameters

\begin{tabular}{|c|c|c|c|c|c|c|c|c|}
\hline Model & $\begin{array}{c}E_{\mathrm{KE}, \text { iso }} \\
\left(10^{52} \mathrm{erg}\right)\end{array}$ & $\begin{array}{c}A_{*} \\
\left(\mathrm{~g} \mathrm{~cm}^{-1}\right)\end{array}$ & $\begin{array}{c}\epsilon_{e} \\
(\%)\end{array}$ & $\begin{array}{c}\epsilon_{B} \\
(\%)\end{array}$ & $\begin{array}{c}\theta \\
\left({ }^{\circ}\right)\end{array}$ & $p$ & $\begin{array}{c}A_{V} \text { (host) } \\
\text { (mag) }\end{array}$ & $\chi_{v}^{2}$ (dof) \\
\hline Wind & $6.8 \pm 0.2$ & $0.56 \pm 0.03$ & $33^{a}$ & $8.1_{-0.7}^{+1.3}$ & $9.6 \pm 0.2$ & $2.11 \pm 0.02$ & $<0.05$ & $0.87(202)$ \\
\hline Wind-Iso $^{\mathrm{b}}$ & $\ldots$ & $\ldots$ & $\ldots$ & $\ldots$ & 90 & $\ldots$ & $\ldots$ & $2.56(203)$ \\
\hline ISM & 185 & 2.5 & $33^{\mathrm{a}}$ & 0.14 & 9.6 & 2.13 & 0 & $1.02(202)$ \\
\hline
\end{tabular}

Notes.

a The electron partition fraction was fixed to its equipartition value.

${ }^{\mathrm{b}}$ For the isotropic model, all parameters are identical to the jetted model, except for the opening angle, which is held fixed.

the peak synchrotron frequency, $v_{m}$, will not fall to the radio bandpass until $t \gtrsim 40$ days. We find that the jet break time is relatively tightly constrained to be $t_{\mathrm{j}}=18.6 \pm 0.8$ days. This is very similar to that found by McBreen et al. (2010). Again, because of the large ratio of $E_{\mathrm{KE} \text {,iso }}$ to $n$, this corresponds to a relatively narrow opening angle: $\theta=\left(3.9_{-0.2}^{+0.2}\right)^{\circ}$.

Correcting for collimation, we find the true energy release from GRB 090902B to be $E_{\gamma}=(7.4 \pm 0.8) \times 10^{51} \mathrm{erg}$, $E_{\mathrm{KE}}=(1.3 \pm 0.3) \times 10^{51} \mathrm{erg}$.

\subsection{GRB 090926A}

\subsubsection{Preliminary Considerations}

Fitting a single power-law decay to the X-ray light curve, we find a reasonable quality fit $\left(\chi^{2}=120.8\right.$ for 97 dof) with $\alpha_{\mathrm{X}}=1.43 \pm 0.03$. The fit quality improves somewhat if we allow for a steeper decay at late times: $\alpha_{X, 1}=1.35 \pm 0.04$, $\alpha_{X, 2}=2.6_{-0.5}^{+0.9}, t_{b}=9.1_{-1.4}^{+2.1}$ days, $\chi^{2}=111.9$ (95 dof), although this in and of itself is not particularly statistically significant. The X-ray spectrum is well fit $\left(\chi^{2}=41.7\right.$ for 51 dof $)$ by a single power law with index $\beta_{\mathrm{X}}=1.12 \pm 0.13$ and does not require any absorption in addition to the Galactic component.

The behavior in the optical bandpass, however, is significantly more complex (Figure 8). The flux in the initial UVOT $U$ - and $V$-band observations declines until $t \approx 0.8$ days, at which point a rebrightening or plateau phase is evident in all five observed filters $(U B V R I)$. The optical light curve of GRB 090926A is reminiscent of the rebrightening seen at $t \approx 1$ day from GRB 970508 (Djorgovski et al. 1997) and GRB 071003 (Perley et al. 2008 and references therein). Such a phenomenon is difficult to reconcile with the standard afterglow paradigm. Possible explanations include a sharp change in the circumburst density (e.g., Lazzati et al. 2002; Tam et al. 2005; but see also Nakar \& Granot 2007) or a smooth injection of energy into the forward shock from the central engine (e.g., Rees \& Meszaros 1998). A more detailed analysis of the early-time behavior of GRB 090926A is beyond the scope of this work.

Simultaneously fitting all of the $B g^{\prime} V r^{\prime} R i^{\prime} I J H K$ data in the range 1 day $<t<10$ days, we find $\alpha_{\mathrm{O}}=1.38 \pm 0.05, \beta_{\mathrm{O}}=$ $0.88 \pm 0.07\left(\chi^{2}=657.6\right.$ for 309 dof $)$. Our derived spectral slope is in good agreement with that found by Rau et al. (2010). However, the temporal decay index we infer is significantly shallower than that reported by both Rau et al. (2010) and Swenson et al. (2010). Again, the discrepancy results largely from how these authors handled deviations from pure powerlaw behavior. Rau et al. (2010) introduced several bumps to their light curve, while Swenson et al. (2010) removed periods of flaring from their fits. We have chosen to instead adopt the simplest power-law models and accept the poorer fit residuals this encompasses. We return to the effect these differences introduce in the derived opening angles and energetics in Section 4.1.

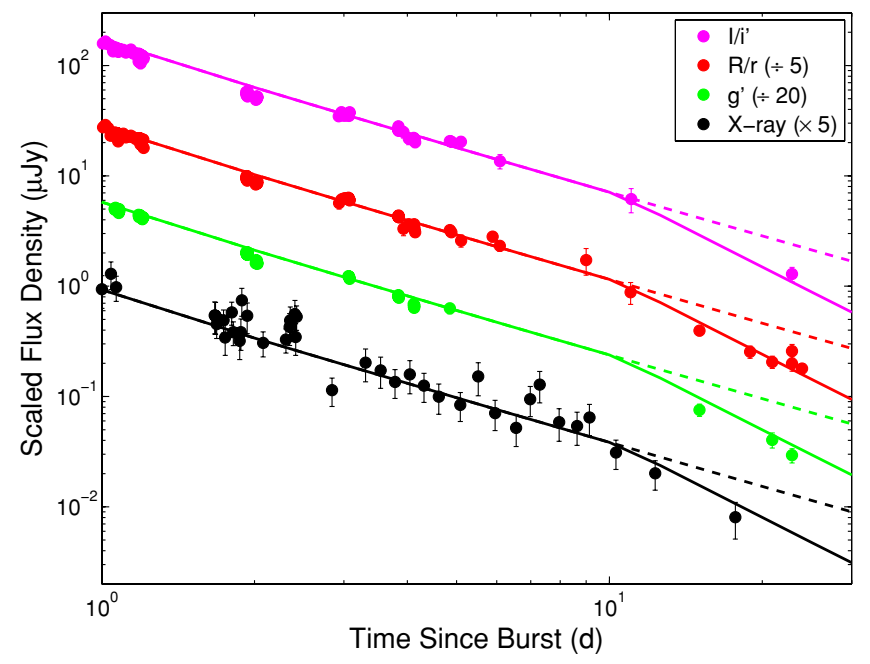

Figure 9. Late-time afterglow and model of GRB 090926A. Both the X-ray and optical $\left(g^{\prime} r^{\prime} i^{\prime}\right)$ bandpasses exhibit a steepening decline at $t \approx 10$ days, strongly indicative of a jet break.

(A color version of this figure is available in the online journal.)

Extrapolating the observed decay indices to our late-time $g^{\prime} r^{\prime} i^{\prime}$ Gemini imaging at $t \approx 23$ days, we find that the flux at late times is significantly overpredicted (Figure 9), suggesting the presence of a break. To quantify this, we fit all of the available $g^{\prime}, r^{\prime}$, and $i^{\prime}$ data at $t>1$ day first to a single power law. The resulting fit is relatively poor, with $\chi^{2}=412.7$ (164 dof). Introducing a simultaneous break in all three filters, we find a significantly improved fit $\left(\chi^{2}=311.3\right.$ for 162 dof; $t_{\mathrm{b}}=10.7 \pm 1.3$ days). The result strongly suggests the presence of a jet break, which we return to discuss in the following section.

Taken together, we find similar temporal $\left(\alpha_{X, 1}=1.35\right.$, $\left.\alpha_{\mathrm{O}, 1}=1.38\right)$ and spectral $\left(\beta_{\mathrm{X}}=1.12, \beta_{\mathrm{O}}=1.03\right)$ indices in the X-ray and optical for $t \approx 1-9$ days. Furthermore, the optical to X-ray spectral index, $\beta_{\mathrm{OX}}$, is comparable to both the optical and X-ray spectral indices. These facts strongly suggest that both the X-ray and optical bandpasses fall in the same synchrotron spectral regime.

Considering the various synchrotron closure relations, the best fit appears to occur when both bandpasses fall above the cooling frequency, $v_{c}$. The afterglow decay in this regime is independent of the circumburst medium. Such a low cooling frequency is somewhat unusual, though not unprecedented (e.g., GRB 050904; Frail et al. 2006), in GRB afterglows. The implied electron index in this case would be $p \approx 2.3$.

\subsubsection{Modeling Results}

Unfortunately, without a radio light curve we cannot uniquely solve for the physical parameters of the afterglow of GRB 090926A. We can, however, at the very least more robustly 
Table 12

Summary of GRB Parameters

\begin{tabular}{lcccccccc}
\hline \hline GRB & $z$ & $\begin{array}{c}f_{\gamma}{ }^{\mathrm{a}} \\
\left(10^{-4} \mathrm{erg} \mathrm{cm}^{-2}\right)\end{array}$ & $\begin{array}{c}E_{\gamma, \text { iso }}{ }^{\mathrm{a}} \\
\left(10^{54} \mathrm{erg}\right)\end{array}$ & $\Gamma_{0}$ & $\begin{array}{c}\theta \\
\left({ }^{\circ}\right)\end{array}$ & $\begin{array}{c}E_{\gamma} \\
\left(10^{51} \mathrm{erg}\right)\end{array}$ & $\begin{array}{c}E_{\mathrm{KE}} \\
\left(10^{51} \mathrm{erg}\right)\end{array}$ & $\begin{array}{c}n / A_{*} \\
\left(\mathrm{~cm}^{-3} / \mathrm{g} \mathrm{cm}^{-1}\right)\end{array}$ \\
\hline 090323 & 3.568 & $1.24 \pm 0.05$ & $3.30 \pm 0.13$ & $\gtrsim 600$ & $2.8_{-0.1}^{+0.4}$ & $4.0_{-0.4}^{+1.2}$ & $1.4_{-0.2}^{+0.6}$ & $0.10 \pm 0.01$ \\
090328 & 0.7357 & $0.68 \pm 0.07$ & $0.096 \pm 0.010$ & $\gtrsim 200$ & $4.2_{-0.8}^{+1.3}$ & $0.26_{-0.11}^{+0.23}$ & $2.2_{-0.9}^{+2.9}$ & $0.26_{-0.07}^{+0.13}$ \\
090902B & 1.8229 & $3.83 \pm 0.05$ & $3.20 \pm 0.04$ & $\gtrsim_{1000}$ & $3.9 \pm 0.2$ & $7.4 \pm 0.8$ & $1.3 \pm 0.3$ & $\left(5.6_{-0.7}^{+0.9} \times 10^{-4}\right.$ \\
090926A & 2.1062 & $1.73 \pm 0.03$ & $1.89 \pm 0.03$ & $\gtrsim 700$ & $9_{-2}^{+4}$ & $23_{-9}^{+26}$ & $\ldots . \mathrm{b}$ & $\ldots$ \\
\hline
\end{tabular}

Notes.

a $1-10^{4} \mathrm{keV}$ observer frame bandpass.

${ }^{b}$ We have not included an estimate for the kinetic afterglow energy or density of GRB 090926A, as these parameters were not well constrained due to the lack of radio data.

constrain the jet break time and opening angle of this event, as well as identify broad trends in the parameters associated with acceptable solutions. As discussed previously, we consider only data at $t \geqslant 1$ day due to the rebrightening observed in the optical before then. To simplify our models and not overly weight the optical bandpass, we have only included observations in the $g^{\prime}$, $r^{\prime}$, and $i^{\prime}$ filters (while this changes the details of the chosen models slightly, it does not alter any of our primary results).

As expected, we were able to find a number of acceptable solutions, both for constant-density and wind-like environments. The best achieved fits for both a wind-like and a constant-density medium are provided in Table 11, with the best-fit wind model plotted in Figure 9. The model provides a reasonable description of the data in all bandpasses, with $\chi^{2}=176.3 .1$ (202 dof). Without radio data, however, the solutions presented in Table 11 are not unique in a global sense, and therefore should only be taken as representative of a larger family of solutions.

Most importantly for our purposes, a jet break at $t \approx$ $10 \pm 2$ days is required to provide a reasonable fit to the latetime optical data (Figure 9). An analogous fit without a jet break results in a significantly worse quality $\left(\chi^{2}=519.7\right.$ for 203 dof $)$. The precise translation into an opening angle is dependent on the circumburst density (both profile and absolute value) and the afterglow energy. Taking the values derived above into account, we find a typical value for a wind-like circumburst medium of $\theta \approx 9^{\circ}$. Based on the observed spread of $E_{\mathrm{KE}}$ and $A_{*}$ in our wind models, we adopt a conservative uncertainty in this value of $\theta \approx\left(9_{-2}^{+4}\right)^{\circ}$.

Correcting for collimation, we find a prompt energy release of $E_{\gamma}=2.3_{-0.9}^{+2.6} \times 10^{52} \mathrm{erg}$. For our best-fit wind model, we find $E_{\mathrm{KE}}=8_{-3}^{+10} \times 10^{50} \mathrm{erg}$, although we note, despite the small statistical uncertainties, that this value is highly ambiguous due to the lack of radio data.

\section{DISCUSSION}

\subsection{Central-engine Constraints I: Energetics and Remnants}

In Table 12, we summarize the primary results from this work, including the redshift, initial Lorentz factor, beaming angle, density, and collimation-corrected energy release for each of the four LAT GRBs considered here.

Before the launch of the Swift satellite in 2004, the majority of all well-observed afterglows were inferred to be highly collimated, with opening angles $\theta \lesssim 10^{\circ}$ (Zeh et al. 2006). The most notable exceptions were the handful of the least distant events, including GRB 980425 (Galama et al. 1998; Kulkarni et al. 1998), GRB 031203 (Soderberg et al. 2004; Sazonov et al. 2004; Thomsen et al. 2004; Cobb et al. 2004; Malesani et al. 2004; Gal-Yam et al. 2004), and (post-Swift) GRB 060218
(Mirabal et al. 2006; Campana et al. 2006; Soderberg et al. 2006; Modjaz et al. 2006; Sollerman et al. 2006; Pian et al. 2006; Ferrero et al. 2006; Maeda et al. 2007), all of which appear to be isotropic explosions that were energetically dominated by their nonrelativistic ejecta (i.e., their associated $\mathrm{SNe}$ ).

The typical GRB afterglows discovered by Swift, however, did not fit neatly into this simple bimodal picture. The afterglows of most Swift GRBs, including the large fraction at cosmological distances, exhibit a much broader range of opening angles (Kocevski \& Butler 2008; Racusin et al. 2009), with some extreme events lacking a detectable jet-break signature in the X-rays out to hundreds of days after the burst (e.g., GRB 060729; Grupe et al. 2007, 2010).

All four of the LAT-detected events we have studied here are consistent with a relatively high degree of collimation $\left(\theta \lesssim 10^{\circ}\right)$. In this respect, then, the afterglows of LAT-detected GRBs more closely resemble the pre-Swift sample. This in and of itself is not entirely surprising, given the tremendous high- $E_{\gamma, \text { iso }}$ bias for events detected by the LAT (Figure 1).

In Figure 10, we plot the two-dimensional (prompt + afterglow) collimation-corrected relativistic energy release for the four LAT events in this work, compared with several additional samples. Shown in red are 11 pre-Swift GRBs at cosmological distances $(z>0.5)$ for which sufficient broadband (X-ray, optical, and radio) data exist to constrain both $E_{\gamma}$ and $E_{\mathrm{KE}}$. We find the logarithmic mean for the sum of these two values, $E_{\text {rel }} \approx E_{\gamma}+E_{\mathrm{KE}}{ }^{28}$ to be $\left\langle E_{\mathrm{rel}}\right\rangle=2.0 \times 10^{51} \mathrm{erg}$, with an error in the mean of $0.17 \mathrm{dex}$. The solid black line indicates a constant value of $E_{\text {rel }}$ corresponding to this mean, while the gray shaded regions indicate $1 \sigma, 2 \sigma$, and $3 \sigma$ confidence intervals.

The gray dotted line in Figure 10 represents a constant gamma-ray efficiency of $\eta_{\gamma}=50 \%$. Most events are roughly consistent with (or even exceed) this value. This result presents a problem for most internal-shock models of the prompt emission, which predict a maximal gamma-ray efficiency of $\eta_{\gamma} \lesssim 10 \%$ (Kobayashi et al. 1997; Daigne \& Mochkovitch 1998). Alternatively, if the early afterglow undergoes a relatively long-lived radiative phase, we may be significantly underestimating the blast-wave kinetic energy (Ghisellini et al. 2010).

As discussed previously, the three most nearby GRBs are clearly subenergetic outliers. The energy release from the $\mathrm{SNe}$ associated with these events, $E_{\mathrm{SN}} \approx 10^{51}-10^{52} \mathrm{erg}$ (Iwamoto et al. 1998; Mazzali et al. 2006), is several orders of magnitude larger than the energy apportioned to the relativistic ejecta. To further distinguish these events, the rate of such subluminous GRBs suggests that they are many times more common (per unit

\footnotetext{
28 Assuming an approximately log-normal distribution, we calculate the weighted mean of $\left\langle\log _{10}\left(E_{\text {rel }}\right)\right\rangle$, where $\sigma=\delta E_{\text {rel }} /\left(\ln (10) \times E_{\text {rel }}\right)$.
} 


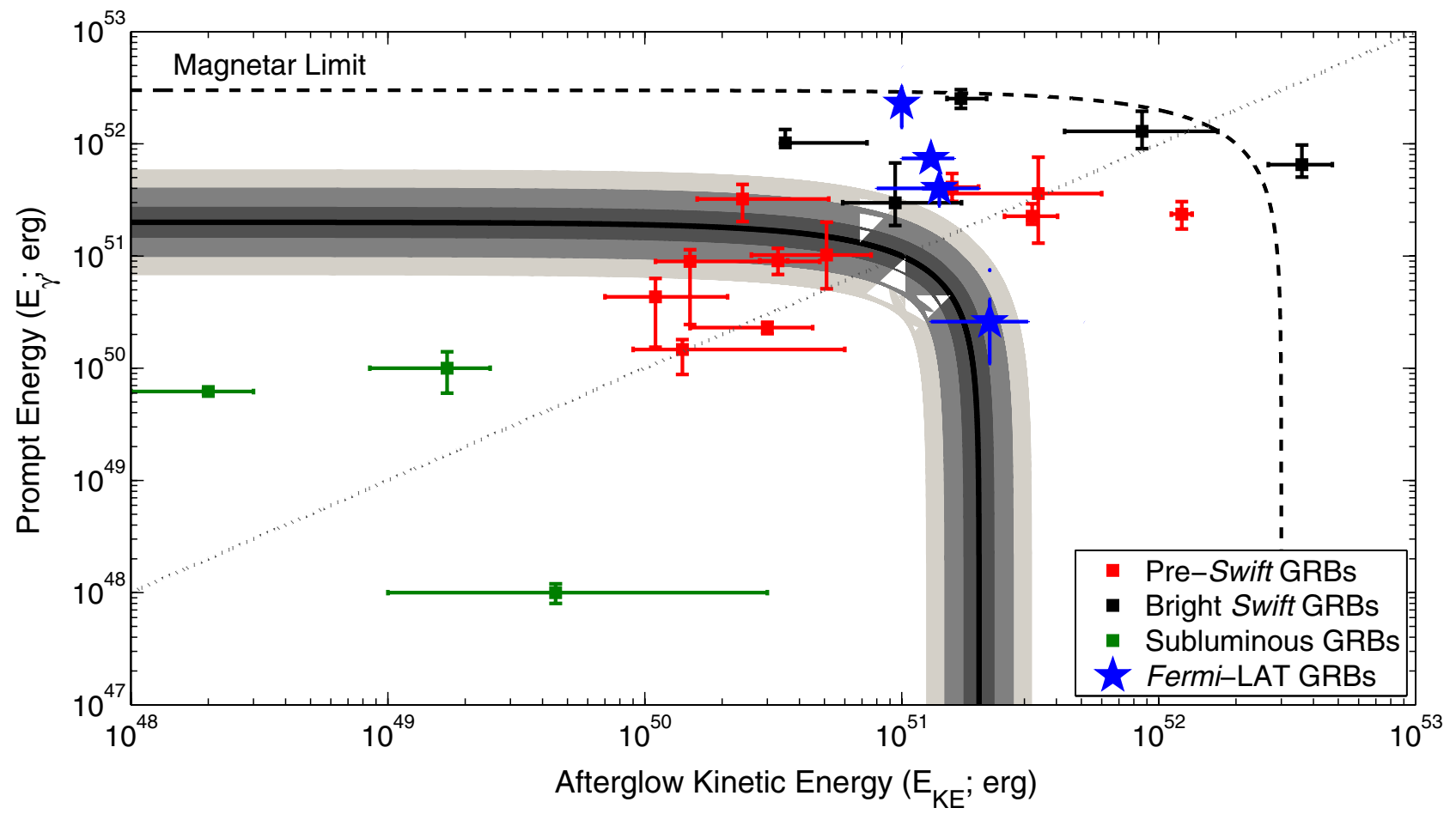

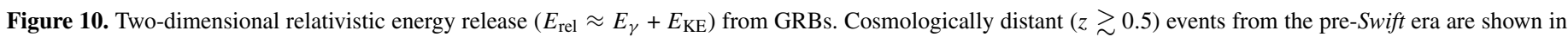

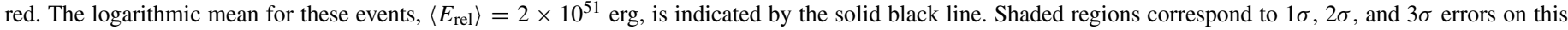

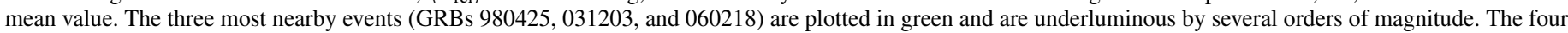

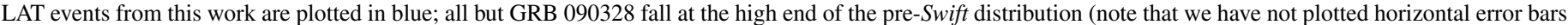

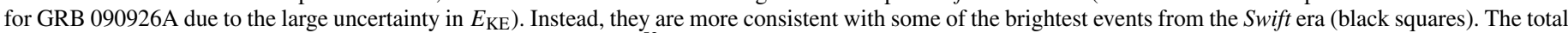

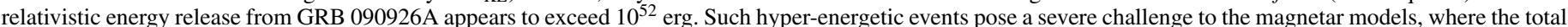

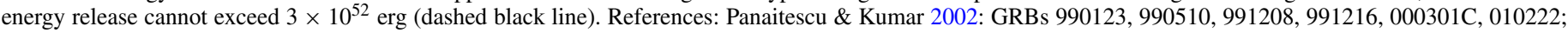

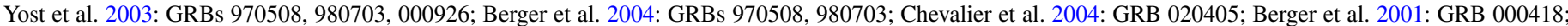

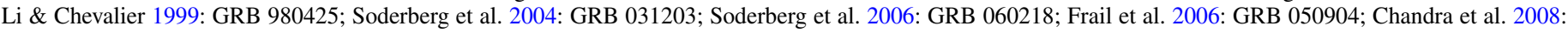
GRB 070125; Cenko et al. 2010: GRBs 050820A, 060418, 080319B.

(A color version of this figure is available in the online journal.)

volume) than the typical cosmological GRBs (Soderberg et al. 2006; Cobb et al. 2006; Guetta \& Della Valle 2007), although Butler et al. (2010) have suggested that both samples may be described by a single luminosity function - that is, subluminous GRBs may simply extend continuously from the higher-energy population.

Of the LAT events studied here, the total relativistic energy output from only a single one (GRB 090328) is consistent with the pre-Swift mean at the $3 \sigma$ level. This is not entirely surprising, however, as the $E_{\text {rel }}$ distribution of pre-Swift GRBs exhibits a reasonable dispersion $(\sim 0.55 \mathrm{dex}$, or a factor of 3.5). It is clear, however, that GRB 090323, GRB 090902B, and GRB 090926A all fall at the very high end of the pre-Swift distribution. In particular, the only event comparable to GRB 090926A, with $E_{\gamma} \approx 10^{52} \mathrm{erg}$ and $E_{\mathrm{KE}}$ relatively unconstrained, is GRB $970508\left(E_{\text {rel }} \approx 1.5 \times 10^{52} \mathrm{erg}\right.$, dominated by the large afterglow kinetic energy; Yost et al. 2003; Berger et al. 2004).

GRBs 090323, 090902B, and 090926A appear consistent instead with a subsample of the brightest Swift events from Cenko et al. (2010; see also Frail et al. 2006; Chandra et al. 2008). This, too, is not unexpected, as these Swift events were chosen on the basis of large $E_{\gamma \text {,iso }}$ values, and therefore should generally mimic at least this component of the LAT selection effects.

Much like several events in the bright Swift sample, GRB 090926A appears to exceed the canonical GRB relativistic energy release of $10^{51}$ erg by roughly an order of magnitude.
We stress that our methodology provides a relatively conservative estimate for the prompt energy release of GRB 090926A, for a number of reasons. First, we consider only the rest-frame $1-10^{4} \mathrm{keV}$ bandpass for all events considered in Figure 10. Extrapolating measurements from previous instruments, with bandpasses typically extending only to $\sim 1 \mathrm{MeV}$ (and just a few hundred $\mathrm{keV}$ for Swift), up to the $\mathrm{GeV}$ range would introduce significant uncertainties in $E_{\gamma, \text { iso }}$. While allowing for a more robust burst-to-burst comparison, we have not included a significant fraction of the detected high-energy fluence in our energy calculations for these LAT events ( 30\% for GRB 090926A).

Second, we have derived the opening angles of all our events using parameters inferred from our broadband modeling. Consequently, the $E_{\text {rel }}$ we derive for GRB 090902B is a factor of several lower than that reported by other authors (Sections 4.3 and 4.4). Most importantly, for GRB 090926A we have deliberately used only wind-like models to derive the beaming angle, even though without radio data it is difficult to differentiate between the two possibilities. For ISM-like models, the opening angles we found were on average somewhat larger, corresponding to a beaming-corrected prompt energy release of $E_{\gamma} \approx 4 \times 10^{52} \mathrm{erg}$.

Finally, we note that our models assume that energy injection into the afterglow has ceased at $t \gtrsim 1$ day. In particular, for GRBs 090323 and 090926A, other authors have attempted to incorporate these effects as late-time bumps in the afterglow light curve (McBreen et al. 2010; Rau et al. 2010). Most notably, 
using this technique for GRB 090926A (for which we have no additional constrains from the radio bandpass), Rau et al. (2010) find no evidence for a jet break out to $t>21$ days. If this is indeed a more appropriate model for the afterglow behavior, our results should be interpreted as a firm lower limit on the opening angle and hence jet break from this event. This would, however, only strengthen the conclusions that follow.

Together with the discovery of several events comparable to GRB 090926A in the past few years, including GRB 050904 $\left(E_{\mathrm{rel}} \approx 2 \times 10^{52} \mathrm{erg} ;\right.$ Frail et al. 2006), GRB $070125\left(E_{\mathrm{rel}} \approx\right.$ $3 \times 10^{52} \mathrm{erg}$; Chandra et al. 2008), GRB 050820A $\left(E_{\mathrm{rel}} \approx\right.$ $4 \times 10^{52} \mathrm{erg}$; Cenko et al. 2010), and GRB $090423\left(E_{\text {rel }} \gtrsim\right.$ $1 \times 10^{52} \mathrm{erg}$; Chandra et al. 2010), we now believe there is substantial evidence in favor of a subpopulation of GRBs with relativistic energy outputs either very near or above $10^{52} \mathrm{erg}$. We refer to such events as hyper-energetic GRBs in what follows, and outline some of the implications of this particular energy threshold.

Much as was argued by Starling et al. (2009) for the case of GRB 080721, the total energy budget is an important diagnostic for any central-engine model. In particular, models for which the outflow is powered by the spindown of a highly magnetized $\left(B \gtrsim 10^{15} \mathrm{G}\right)$ proto-neutron star are subject to strict constraints on the total energy budget of $E_{\text {total }}<3 \times 10^{52} \mathrm{erg}$ (the rotational energy of a maximally spinning $1.4 M_{\odot}$ neutron star; e.g., Thompson et al. 2004; Metzger et al. 2007).

Only accounting for the relativistic energy output, GRB 090926A seems to approach within a factor of a few of this limit. While we caution that there are still significant uncertainties associated with the models used to infer the afterglow parameters (and hence $E_{\text {rel }}$ ), we have not yet accounted for additional sources of energy, including (nonrelativistic) SN emission, radiative losses at early times due to bright X-ray flares (Burrows et al. 2005a; Falcone et al. 2007), and synchrotron losses during the later phases of afterglow evolution (Yost et al. 2003). Even if we allow for a slightly more massive neutron star (e.g., Demorest et al. 2010), the tremendous efficiency required by this process strains credulity.

\subsection{Central-engine Constraints II: Lorentz Factor, Opening Angle, and Acceleration Mechanisms}

Unlike magnetar models that are powered by the spindown of a highly magnetized proto-neutron star, GRB models in which the core of an evolved massive star collapses promptly to form a black hole and accretion-disk system ("Type I collapsars" following the nomenclature of MacFadyen et al. 2001) have significantly relaxed constraints on the total energy budget that are in at least some cases capable of accommodating hyperenergetic events. For instance, the initial models of MacFadyen \& Woosley (1999) that begin with a $35 M_{\odot}$ He star with a $10 M_{\odot}$ evolved core lead to accretion rates of $\sim 0.1 M_{\odot} \mathrm{s}^{-1}$ onto a $3 M_{\odot}$ rotating black hole. Assuming approximately continuous feeding over the fallback time of the stellar envelope $(\sim 10 \mathrm{~s})$, this corresponds to a total accreted mass $\sim 1 M_{\odot}$. In addition to the accretion process, an even larger reserve lies in the rotational energy of the black hole:

$$
E_{\mathrm{rot}}=\frac{M_{\mathrm{BH}} c^{2}}{2}\left\{2-\left[\left(1+\sqrt{1-a^{2}}\right)^{2}+a^{2}\right]^{1 / 2}\right\},
$$

where $M_{\mathrm{BH}}$ is the black hole mass and $a$ is the dimensionless rotation parameter $\left(a \equiv J c / M_{\mathrm{BH}}^{2} G\right)$.
At least two competing theories exist, however, to explain how the system can channel this energy to produce the collimated, relativistic jets we observe from GRBs. First, the energy may be extracted from the accretion process via $\nu \bar{v}$ annihilation (e.g., Woosley 1993; MacFadyen \& Woosley 1999; Popham et al. 1999; Ruffert \& Janka 1999; Narayan et al. 2001). At the hyper-Eddington rates expected for collapsars, the accretion disk formed around the rotating black hole will be optically thick and photons are unable to escape. The viscous heat is instead balanced by cooling via neutrino emission. Annihilation of $v \bar{v}$ pairs will produce a gas of hot electron-positron pairs, which, assuming sufficiently low baryon loading, can then rapidly expand in the low-density regions along the axis of rotation (a "fireball"; e.g., Zhang et al. 2003).

Alternatively, if the black hole is rotating and the accretion disk is threaded by sufficiently large magnetic fields ( $B \approx 10^{15} \mathrm{G}$ for a $3 M_{\odot}$ black hole), energy can be extracted directly from the rotating black hole via the Blandford-Znajek mechanism (e.g., Blandford \& Znajek 1977; Lee et al. 2000). The accretion disk serves primarily to anchor the strong magnetic field (which would otherwise disperse), and the energy is emitted as a largely Poynting-flux-dominated outflow.

The efficiency of converting the potential energy of the rotating black hole plus accretion-disk system into a form suitable to launch a collimated, relativistic outflow has been intensively studied in the last decade. In the case of $v \bar{v}$ annihilation, the overall efficiency of converting the neutrino luminosity of the cooling disk $\left(L_{\nu}\right)$ into $e^{+} e^{-}$pairs $\left(L_{\nu \bar{v}}\right)$ depends sensitively on the mass accretion rate, the disk geometry, and (perhaps) the effects of general relativity on neutrino physics (e.g., Popham et al. 1999; MacFadyen \& Woosley 1999; Ruffert \& Janka 1999). But the majority of recent simulations suggest at most a modest efficiency $\left(\eta_{\nu \bar{v}} \equiv L_{\nu \bar{v}} / L_{\nu} \lesssim 0.5 \%\right)$ for the energy available to launch a relativistic jet (e.g., Rosswog et al. 2003; Birkl et al. 2007; Harikae et al. 2010). For typical disk luminosities of $L_{v} \approx 10^{51}-10^{52} \mathrm{erg} \mathrm{s}^{-1}$, this would require an extremely long-lived phase of continuous accretion to explain hyper-energetic GRBs.

Jets powered by MHD processes, on the other hand, can more easily accommodate large energy releases. To begin with, by extracting energy directly from the rotation of the black hole, the Blandford-Znajek process begins with a significantly larger energy reservoir: $E_{\text {rot }} \approx 8 \times 10^{53} \mathrm{erg}$ for a rapidly spinning $(a=0.9)$ black hole of mass $3 M_{\odot}$ (Equation (1)). Both analytic models (e.g., Lee et al. 2000) and numerical simulations (e.g., McKinney 2005) suggest that as much as $5 \%-10 \%$ of this rotational energy can be made available to power a collimated outflow, easily within the requirements of hyper-energetic events. We caution, however, that the jet efficiency is a strong function of the black hole spin, and slowly spinning black holes have significantly lower efficiencies.

Setting energetics considerations aside for the moment, there still remains the question of whether either the $v \bar{v}$ annihilation mechanism or MHD processes are capable of producing jets with the Lorentz factors and degree of collimation inferred from GRB observations. Here our sample of Fermi-LAT events, with their extreme initial Lorentz factors, offers a distinct advantage over previous GRB studies. Combining the Lorentz-factor limits for the most relativistic GRBs with the inferred jet opening angles from our broadband afterglow models can provide critical diagnostics of the jet acceleration mechanism.

The results from MHD simulations of jet acceleration appear to depend sensitively on the nature of the medium into which 
the jet propagates. Tchekhovskoy et al. (2010) have recently conducted fully relativistic MHD simulations in which a mildly magnetized (magnetization parameter $\sigma \lesssim 1$ ) jet is initially confined by the pressure of a stellar envelope out to some radius, and then allowed to propagate freely in all directions (see also Komissarov et al. 2010). Both authors find that the jet accelerates rapidly in this transition region ("rarefaction" acceleration) and can reach Lorentz factors $\Gamma_{0}$ of a few hundred, yet still remains highly collimated $\left(\theta \lesssim 5^{\circ}\right)$ even after leaving the region of confinement. Furthermore, the deconfinement radii required to produce these outflows agree well with the expected value for Wolf-Rayet stars $\left(r \approx 10^{9}-10^{11} \mathrm{~cm}\right)$.

Based on both results from these simulations and analytical arguments, Tchekhovskoy et al. (2010) demonstrate a relationship between the initial Lorentz factor and jet opening angle: $\Gamma_{0} \theta \approx 10-30$. This result is able to nicely reproduce the typical observed Lorentz factors (Lithwick \& Sari 2001) and opening angles (Zeh et al. 2006) of previous GRB samples. However, for the events studied here, both GRB 090902B $\left(\Gamma_{0} \theta \approx 70\right)$ and GRB 090926A $\left(\Gamma_{0} \theta \approx 110\right)$ appear inconsistent with this result.

One way to circumvent this requirement is if the jets have extremely high magnetization parameters $(\sigma \gg 1$; Tchekhovskoy et al. 2009), as such Poynting-flux-dominated jets can be accelerated to extreme Lorentz factors over relatively large angles. However, it is unclear how such outflows can convert sufficient electromagnetic energy to accelerate electrons and produce the observed prompt gamma-ray emission. Other particleacceleration mechanisms besides MHD shocks may be required in this case (e.g., Beloborodov 2010). Alternatively, the gammaray emission may be patchy (e.g., Kumar \& Piran 2000) or the jet may be structured (see, e.g., Granot 2007 and references therein), so that we are measuring only the extrema of $\Gamma_{0}$ and not the true bulk of the relativistic flow carrying most of the energy.

\subsection{Comparison with Other Work}

In this section, we attempt to place this work in context, both by comparing our results with those of other authors who have studied these same events and by highlighting additional differences between our LAT sample and GRBs detected by satellites at lower energies. As we have (for the most part) previously discussed the results from different model fits to the data in Section 3, we focus here on the implications for these events.

McBreen et al. (2010) present optical and NIR observations of three events from this work (GRBs 090323, 090328, and 090902B), taken primarily with the GROND instrument (Greiner et al. 2008). In the case of GRB 090323, McBreen et al. (2010) infer from the steep optical decay that a jet break occurred before the first optical observations began $\left(t_{\mathrm{j}} \lesssim 1\right.$ day), resulting in a narrow beaming angle $\left(\theta \lesssim 2^{\circ}\right)$ and a correspondingly small collimation-corrected prompt energy release $\left(E_{\gamma} \lesssim 3(1) \times 10^{51}\right.$ erg for a constant-density (wind-like) circumburst medium). We consider this possibility unlikely, however, as it is difficult to explain both the flat radio light curve and the more slowly fading $\mathrm{X}$-ray afterglow $\left(\alpha_{\mathrm{X}} \approx 1.5\right)$ through post-jet-break evolution (see also Section 4.4).

These authors arrive at a similar conclusion regarding the jet break of GRB 090328, inferring $t_{\mathrm{j}} \lesssim 1.5$ days. However, it is again difficult to reconcile the shallower X-ray decay $\left(\alpha_{\mathrm{X}} \approx 1.65\right)$ and in particular the rising radio light curve at this time with models of post-jet-break evolution (see also Section 4.4).
We find very similar results to those of McBreen et al. (2010) for the temporal and spectral indices, inferred circumburst medium, and jet break time for GRB 090902B. In particular, the late-time $(t \approx 23$ days) optical observation with the VLT provides strong confirmation that the radio decline at $t \gtrsim 6$ days is due to a jet break. However, our results diverge when translating the jet break time into an opening angle. Because of the low density we derive for the circumburst medium of GRB 090902B, a jet break time of $t_{\mathrm{j}} \approx 18$ days corresponds to a relatively narrow opening angle. The resulting constraints on $E_{\gamma}$ are roughly a factor of three less (note that part of this difference is also due to the wider bandpass McBreen et al. 2010 use to calculate $\left.E_{\gamma, \text { iso }}\right)$.

Pandey et al. (2010) also present optical and X-ray observations of GRB 090902B. The afterglow model derived by these authors is broadly similar to ours, but we favor a somewhat steeper electron index ( $p=2.2$ versus 1.8$)$. Most importantly, Pandey et al. (2010) derive a limit on the opening angle of $\theta>6^{\circ}$ (based on a lower limit to the jet break time of $t_{\mathrm{j}}>6$ days). Though our derived jet break time is consistent with the results of these authors, our inferred opening angle is a factor of 1.5 smaller due to the lower value we have used for the circumburst density. As a result, the limits we derive for the prompt gamma-ray energy release are a factor of a few less.

Kumar \& Barniol Duran (2010; see also Kumar \& Barniol Duran 2009) have also presented an analysis of the broadband afterglow of GRB 090902B. Interestingly, these authors suggest that the delayed high-energy ( $E \gtrsim 100 \mathrm{MeV}$ ) component observed from several LAT events is in fact due to the same source as the late-time afterglow emission: synchrotron radiation from accelerated electrons in the circumburst medium shock-heated by the outgoing blast wave (i.e., external-shock emission; see also Ghisellini et al. 2010).

The afterglow parameters derived by these authors for GRB 090902B differ somewhat from ours, largely due to the fact that they assume that the X-ray bandpass at $t \approx 1$ day falls below the synchrotron cooling frequency (i.e., $v_{\mathrm{X}}<v_{c}$ ). While the observed X-ray spectral and temporal indices at $t \approx 1$ day are consistent with this picture, we find that a better fit can be achieved for both the X-ray and optical data if $v_{\mathrm{X}}>v_{c}$ at $t \approx 1$ day (see also Pandey et al. 2010).

Using the best-fit late-time afterglow parameters we have derived for GRB 090902B (Table 10), we have attempted to calculate the magnitude of the afterglow (i.e., external shock) flux at $v=100 \mathrm{MeV}$ and $t=50 \mathrm{~s}$. We find $f_{v} \approx 10 \mathrm{nJy}$, roughly a factor of 10 below the observed value (Abdo et al. 2009a). The discrepancy between our results and those of Kumar \& Barniol Duran (2010) is largely due to the lower kinetic energy we have inferred for the blast wave $\left(E_{\mathrm{KE} \text {,iso }} \approx 6 \times 10^{53}\right.$, a factor of 20 lower than that used by Kumar \& Barniol Duran 2010). This would suggest that the delayed high-energy component is not due to afterglow emission. We caution, however, that our flux calculation does not incorporate the reduced cross section for inverse-Compton emission due to the Klein-Nishina effect at very high energies, and this will affect our flux calculation to some extent.

A further interesting claim from the work of Kumar \& Barniol Duran (2010) is that the inferred value of the magnetic field for GRB 090902B (along with several other LAT-detected events) is consistent with shock compression of a modest circumstellar field ( $B \gtrsim 30 \mu \mathrm{G}$; see also Piran \& Nakar 2010). In other words, no dynamo process is necessary to generate the magnetic field strengths needed to produce the observed synchrotron 


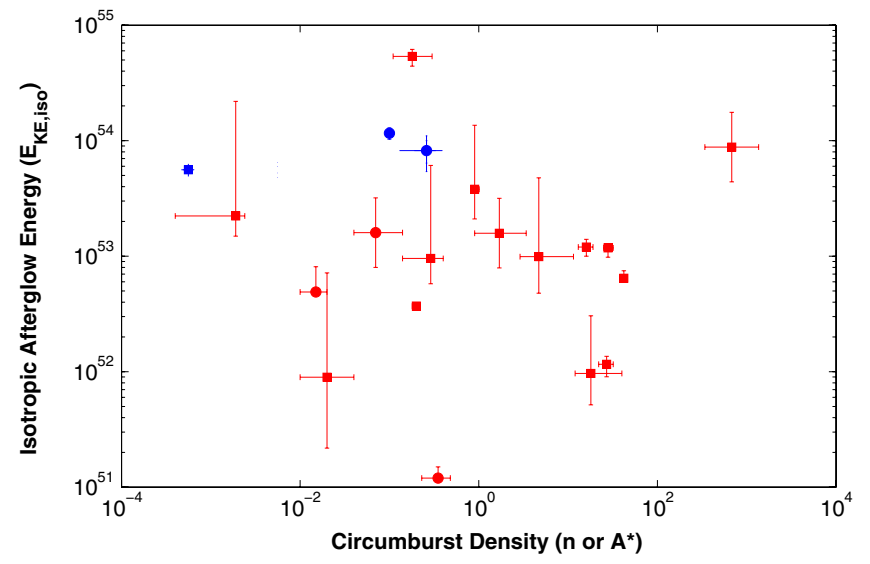

Figure 11. Circumburst density and isotropic afterglow energy release from cosmological $(z>0.5)$ GRBs. Constant-density models are shown as squares, while wind-like models are plotted as circles. The GRBs detected by the FermiLAT (blue) clearly have preferentially larger isotropic energy releases and smaller circumburst densities than the rest of the sample. See the caption of Figure 10 for references.

(A color version of this figure is available in the online journal.)

afterglow emission. For a constant-density circumburst medium, the preshock magnetic field is given by

$$
B=\left(2 \pi m_{p} \epsilon_{B} n\right)^{1 / 2} c .
$$

We also find that the product $\epsilon_{B} \times n$ (and hence the derived $B$ field) is smaller for LAT events than for other GRBs detected at lower energies, primarily due to the lower inferred circumburst densities (see below). However, using our best-fit parameters for GRB $090902 \mathrm{~B}$, we find $B \approx 1 \mathrm{mG}$. While this value is smaller than the preshock magnetic fields previously inferred for GRBs $\left(B \approx 10 \mathrm{mG}\right.$ for $\left.\epsilon_{B}=0.01, n=1 \mathrm{~cm}^{-3}\right)$, it suggests that at least some magnetic-field amplification may still be required.

Finally, we return to the issue of the relatively low circumburst densities derived in Section 3. Given the strong evidence for a connection between long-duration GRBs and broad-lined SNe $\mathrm{Ib} / \mathrm{c}$, it would be natural to expect the massive-star progenitors of GRBs to explode in the dense molecular cloud environments where we observe stars forming in our own Galaxy.

Past modeling of broadband GRB afterglows, however, has not always revealed this to be the case. Of the past GRBs with sufficient radio observations to estimate the circumburst density, the derived values span a large range from $1.9 \times 10^{-3} \mathrm{~cm}^{-3}$ (GRB 990123; Panaitescu \& Kumar 2002) to $680 \mathrm{~cm}^{-3}$ (GRB 050904; Frail et al. 2006). We plot in Figure 11 the derived circumburst densities for these previous events (either $n$ or $A_{*}$, depending on the circumburst density profile) as a function of isotropic afterglow kinetic energy, along with three events from our LAT sample (we have not included GRB 090926A as lack of radio coverage makes density estimates highly degenerate). It is clear that the events detected by the LAT have on average larger isotropic energies and smaller densities than the previous sample.

The larger isotropic kinetic energy is not hard to understand, as the LAT is less sensitive than, for example, the Burst Alert Telescope (Barthelmy et al. 2005) on board Swift and should therefore select brighter events (in terms of the high-energy fluence). Unless $E_{\gamma, \text { iso }}$ and $E_{\mathrm{KE} \text {,iso }}$ were anticorrelated, we would expect LAT events to have larger isotropic blast-wave energies as well. But the lower densities require an additional explanation. We speculate on possible reasons for this difference in Section 5.
The large ratio of $E_{\mathrm{KE} \text {,iso }} / n$ (or, alternatively $E_{\mathrm{KE} \text {,iso }} / A_{*}$ ) we have found for these events has two important implications. First, the opening angle derived for a given jet break time scales inversely as $E_{\mathrm{KE} \text {,iso }}$ and proportionally to $n / A_{*}$ (Section 4.4). Thus, for a given jet break time, the large ratios we have inferred for LAT events imply smaller opening angles than were one to simply use a canonical value (e.g., $n=0.1 \mathrm{~cm}^{-3}$ from Frail et al. 2001; $n=10 \mathrm{~cm}^{-3}$ from Bloom et al. 2003). In this manner, LAT events can have relatively late jet breaks but still be narrowly collimated $\left(\theta \lesssim 10^{\circ}\right)$.

Second, a large $E_{\mathrm{KE}, \text { iso }} / n$ ratio will act to delay the deceleration time of the outflow. Assuming the "thin shell" case, the outgoing relativistic blast wave will decelerate when (Sari \& Piran 1999; Molinari et al. 2007)

$$
\begin{gathered}
t_{\mathrm{dec}}(\mathrm{ISM})=\left(\frac{3 E_{\mathrm{KE}, \text { iso }}(1+z)^{3}}{32 \pi m_{p} c^{5} n \Gamma_{0}^{8}}\right)^{1 / 3}, \\
t_{\mathrm{dec}}(\text { Wind })=\frac{E_{\mathrm{KE}, \text { iso }}(1+z)}{8 \pi c^{3} A \Gamma_{0}^{4}} .
\end{gathered}
$$

(Note that we have modified Equation (2) from Molinari et al. 2007 , as these authors use an alternative definition of $A$.) To some extent, the large ratio of $E_{\mathrm{KE} \text {,iso }} / n$ will offset the effect of the larger initial Lorentz factors for Fermi-LAT events. Using the parameters we have derived from the LAT events in our sample, we find $t_{\mathrm{dec}} \approx 2 \mathrm{~s}$ for GRB 090323, $t_{\mathrm{dec}} \approx 3 \mathrm{~s}$ for GRB 090328, and $t_{\mathrm{dec}} \approx 33 \mathrm{~s}$ for GRB 090902B. If the latetime $\mathrm{GeV}$ emission is indeed due to external shock emission, the delay between the $\mathrm{MeV}$ and $\mathrm{GeV}$ photons should correspond roughly to the blast-wave deceleration time. This may be problematic for GRB 090902B, for which the observed delay $(\sim 4 \mathrm{~s})$ is significantly less than our inferred deceleration time. We caution, however, that the large systematic uncertainties in $E_{\mathrm{KE} \text {,iso }}, n$, and particularly $\Gamma_{0}($ Section 4.4$)$ may introduce a significant error into calculations of the deceleration time.

\subsection{Limitations and Future Work}

To better understand our results in Sections 4.1, 4.2, and 4.3 on GRB energetics and circumburst density, it is important to consider the robustness of our modeling. The most crucial factor in determining the true energy release is the collimation correction, which we therefore examine in more detail here.

For a wind-like circumburst medium, the jet half-opening angle $\theta$ can be written as (e.g., Chevalier \& Li 2000)

$$
\begin{aligned}
\theta(\text { Wind })= & 0.10\left(\frac{1+z}{2}\right)^{-1 / 4}\left(\frac{E_{\mathrm{KE}, \text { iso }}}{10^{52} \mathrm{erg}}\right)^{-1 / 4}\left(\frac{A_{*}}{1.0 \mathrm{~cm}^{-1}}\right)^{1 / 4} \\
& \times\left(\frac{t_{\mathrm{j}}}{1 \text { day }}\right)^{1 / 4}(\mathrm{rad}) .
\end{aligned}
$$

This result assumes only that the shock is ultrarelativistic and undergoes a self-similar evolution (Blandford \& McKee 1976), and is therefore relatively robust.

Only in the case of GRB 090926A (and also for GRB 090902B if we include the late-time VLT observation from McBreen et al. 2010) do we find clear evidence for a jet break in multiple bandpasses. For GRB 090323, the lack of a steepening in the X-ray and optical light curves apparently limits $t_{\mathrm{j}} \gtrsim 10$ days. If we simply ignore our modeling results and assume that the shock energy is comparable to the prompt 
gamma-ray energy $\left(E_{\mathrm{KE} \text {,iso }} \approx 4 \times 10^{54} \mathrm{erg}\right)$ and that the progenitor wind speed and mass-loss rate are comparable to those observed from Galactic Wolf-Rayet stars $\left(A_{*} \approx 1 \mathrm{~g} \mathrm{~cm}^{-1}\right)$, then we find a lower limit on the opening angle of $\theta$ (Wind) $\gtrsim 3^{\circ}$. The resulting limit on the prompt energy release is therefore $E_{\gamma} \gtrsim 5 \times 10^{51} \mathrm{erg}$, similar to what we derived from our broadband models.

If we were instead to assume the GRB exploded in a constantdensity medium, the opening angle then becomes (e.g., Sari et al. 1999)

$$
\begin{aligned}
\theta(\mathrm{ISM})= & 0.12\left(\frac{1+z}{2}\right)^{-3 / 8}\left(\frac{E_{\mathrm{KE}, \text { iso }}}{10^{52} \mathrm{erg}}\right)^{-1 / 8}\left(\frac{n}{1 \mathrm{~cm}^{-3}}\right)^{1 / 8} \\
& \times\left(\frac{t_{\mathrm{j}}}{1 \mathrm{day}}\right)^{3 / 8}(\mathrm{rad}) .
\end{aligned}
$$

Under similar assumptions as above, the limit on the opening angle would be $\theta$ (ISM) $\gtrsim 6^{\circ}$, corresponding to a prompt energy release of $E_{\gamma} \gtrsim 2 \times 10^{52} \mathrm{erg}$. Clearly a constant-density circumburst medium only makes the energy requirements more strict.

An alternative possibility for GRB 090323 (McBreen et al. 2010; also suggested by Schady et al. 2007 to explain the afterglow of GRB 061007) is a jet break before the beginning X-ray and optical observations ( $t \lesssim 1$ day for GRB 090323). Much like GRB 061007, however, the implied post-break decay index in the X-rays $\left(\alpha_{X} \approx 1.5\right)$ is too shallow to accommodate a typical electron spectral index $p$ (for $v>v_{m}, f_{v} \propto t^{-p}$ post-jet-break; Sari et al. 1999). The observed behavior would therefore require late-time energy input to flatten the X-ray and optical decay. Furthermore, the relatively flat radio light curve for $t \lesssim 20$ days would require that the synchrotron selfabsorption frequency fell above the radio bandpass during this phase; otherwise the radio bandpass would be required to decay, either as $t^{-1 / 3}\left(v<v_{m}\right)$ or $t^{-p}\left(v>v_{m}\right){ }^{29}$ We consider this somewhat contrived picture unlikely in the case of GRB 090323, particularly given the reasonable quality of our fits using only standard afterglow theory.

A similar analysis of GRB 090328 suggests that the jet-break time cannot occur any earlier than $t \approx 3$ days, based again on the relatively steady decay seen in the X-ray light curve and, in particular, on the rising radio emission at early times. The corresponding limits on the opening angles are therefore $\theta$ (Wind) $\gtrsim 8^{\circ}, \theta$ (ISM) $\gtrsim 7^{\circ}$. Both values result in a prompt energy release $E_{\gamma} \gtrsim 10^{51} \mathrm{erg}$, broadly consistent with our modeling results.

In the end, all of these estimates of energy, density, and jet geometry rest on the relativistic synchrotron model. While the standard afterglow model has undergone continuous improvements and it has been well tested (Meszaros \& Rees 1997; Sari et al. 1998, 1999; Wijers \& Galama 1999; Chevalier \& Li 2000; Panaitescu \& Kumar 2000; Yost et al. 2003; Willingale et al. 2007), it still must make several simplifying assumptions about the shock dynamics, magnetic-field generation, particle acceleration, energy injection, and the circumburst density structure.

One approach to improve our estimates of the relativistic energy output involves better numerical simulations of the shock hydrodynamics. Recent results from both Zhang \& MacFadyen

\footnotetext{
29 We note that the above results are independent of the circumburst medium, as the expansion of the outflow is predominantly lateral after the jet break (Chevalier \& Li 2000).
}

(2009) and van Eerten et al. (2010) suggest that viewing-angle effects, in particular observing lines of sight that are offset with respect to the outflow direction (but still inside the jet opening angle), can lead to overestimates of the energy budget by factors of a few. Given the tremendous Lorentz factors implied by the detection of $\mathrm{GeV}$ photons, it seems that offaxis effects should be more important for typical Swift GRBs, where relativistic beaming during the prompt emission is not nearly as severe, and play less of a role for Fermi-LAT events. Furthermore, competing geometric effects may actually serve to postpone the jet break time, particularly in the radio bands, leading to underestimates of the total explosion energy by factors approaching two (van Eerten et al. 2011).

Nonetheless, it is clear that if we are to verify this class of hyper-energetic bursts, we need independent estimates of the relativistic energy content. Waxman et al. (1998) first suggested that late-time (radio) calorimetry could be used for this purpose in order to sidestep early-time complications such as the outflow geometry, the density structure, and ongoing activity from the central engine. Recent relativistic hydrodynamic simulations show that the earlier analytic models which used spherical geometry and Sedov-Taylor dynamics to explain the late-time behavior were approximately correct and produce a robust estimate of the total kinetic energy (Zhang \& MacFadyen 2009). In the past, the method has been limited to only a small number of radio-bright events (e.g., van der Horst et al. 2008, and references therein). But it is somewhat encouraging that both early- and late-time estimates for the most energetic pre-Fermi event (GRB 970508; $E_{\mathrm{KE}} \approx 10^{52} \mathrm{erg}$ ) are in close agreement (Yost et al. 2003; Berger et al. 2004). With the advent of radio facilities having greatly increased sensitivity such as LOFAR (van Haarlem 2005) and the EVLA (Perley et al. 2009c), it should soon be possible to verify candidate hyperenergetic events by searching for long-lived $(t \gtrsim 100$ days) radio afterglows.

Finally, we add a further note of caution that the derivation of the initial Lorentz factor $\Gamma_{0}$ is also subject to significant systematic uncertainties (e.g., Bošnjak et al. 2009). In order to calculate the optical depth to pair production, it is necessary to determine the effective blast-wave radius as well as the instantaneous spectral parameters. The former is typically inferred from the variability timescale, while the latter is usually averaged over the entire duration of the prompt emission, and it is not always clear how large an effect this will have on the calculation of $\Gamma_{0}$. The calculations are further complicated if the spectrum is not a pure power law, as would be the case for thermal emission arising from the jet photosphere (Ryde et al. 2010). Here we have attempted to calculate the initial Lorentz factors in a consistent manner, and it is not unreasonable to believe that the relative ordering of $\Gamma_{0}$ is robust. However, small changes in $\Gamma_{0}$ can have a large effect on some of the parameters derived here (e.g., $t_{\mathrm{dec}}$; Equations (3) and (4)). Future early afterglow observations of Fermi-LAT GRBs to directly measure the deceleration time and hence constrain $\Gamma_{0}$ (e.g., Molinari et al. 2007) could provide valuable insight into this area.

\section{CONCLUSIONS}

We have undertaken extensive broadband continuum (radio, optical, and X-ray) and spectroscopic observations of four long-duration GRBs (GRBs 090323, 090328, 090902B, and 090926A) detected by the LAT instrument on the Fermi satellite at $\mathrm{GeV}$ energies. This work was motivated by the realization that Fermi is especially sensitive to GRBs with large isotropic 
energy release, and hence provides an interesting sample of events to test GRB central-engine models and their relativistic outflows. Our afterglow models constrain the jet break times and the density of the circumburst medium, from which we derive the collimation angle and hence the beaming-corrected energy release for each event. We find three GRBs with a total relativistic content about an order of magnitude in excess of the canonical $10^{51} \mathrm{erg}$, with GRB 090926A almost certainly in excess of $10^{52} \mathrm{erg}$. This analysis provides support for our earlier claim of a class of hyper-energetic GRBs. The discovery of more GRBs with total energy release $\gtrsim 10^{52} \mathrm{erg}$ is troubling for central engines in which the energy to drive the jet is derived either from a rotating magnetar or collapsars powered by neutrino annihilation. For this reason, we are led to believe that, at least for hyper-energetic GRBs, the massive star progenitor collapses directly to a black hole and the rotational energy of this system is extracted via the Blandford-Znajek process.

Although we find relatively narrow opening angles for all four events $\left(\theta \lesssim 10^{\circ}\right)$, the extreme initial Lorentz factors inferred for these LAT events imply that the product $\theta \Gamma_{0}$ can be a factor of 5-10 larger than estimates of previous GRBs detected at $\mathrm{MeV}$ energies. These values are inconsistent with recent simulations of low-magnetization MHD jets, suggesting that the outflow may be at least initially Poynting-flux dominated. If this is indeed the case, it is unclear how the initial kinetic energy of the outflow is converted to prompt gamma-ray emission.

Interestingly, for the three events having sufficient radio coverage to derive a circumburst density, we find anomalously large values of $E_{\mathrm{KE} \text {,iso }} / n$ (or, for a wind-like medium, $E_{\mathrm{KE} \text {,iso }} / A_{*}$ ). While the large $E_{\mathrm{KE} \text {,iso }}$ values are simple to understand, the low circumburst densities require a more complex explanation.

One possibility is that the progenitor stars of LAT GRBs are somehow different from the progenitors of most previous GRBs detected at $\mathrm{MeV}$ or $\mathrm{keV}$ energies. It is currently thought that GRB progenitors are distinguished from the progenitors of ordinary $\mathrm{SNe} \mathrm{Ib} / \mathrm{c}$ by their low metallicities (e.g., MacFadyen \& Woosley 1999; Woosley \& Heger 2006; Modjaz et al. 2008): the lower mass-loss rates allow the progenitors of GRBs to keep more angular momentum. The increased rotation evacuates a cavity through which a relativistic jet can propagate. If LAT events have larger initial Lorentz factors, it may be that they come from lower-metallicity progenitors with minimal pre-explosion mass loss. In fact a recent measurement of the metallicity of the host galaxy of GRB $090926 \mathrm{~A}, \log \left(Z / \mathrm{Z}_{\odot}\right) \approx$ -1.9 , represents one of the lowest values ever found for a GRB system, although the matter probed by these observations may lie at sufficiently large distances to be uncorrelated with progenitor properties (Rau et al. 2010). Observations of additional host galaxies of these events, both through absorption and emission spectroscopy, may help shed light on this matter.

It is also possible that the low-density preference is the result of other, more subtle, selection effects. In particular, if the $\mathrm{GeV}$ emission arises in the external shock, the Fermi-LAT could be biased toward events in low-density environments. If the circumburst density is too high, the blast wave will decelerate at small radii (Equations (3) and (4)), where the outflow may be opaque to $\mathrm{GeV}$ photons. More observations of LAT GRBs, particularly at very early times, would help to investigate this hypothesis.

We end by emphasizing the importance of afterglow observations of high- $E_{\gamma \text {,iso }}$ events in the Fermi era to provide further confirmation of this picture. Such GRBs are either highly collimated outflows $\left(\theta \lesssim 2^{\circ}\right)$ with a typical energy release, or truly hyper-energetic events; both represent extreme tests of jet collimation and central-engine models, respectively. Current efforts suffer from delays in LAT localizations and limited groundbased afterglow follow-up efforts. The latter can be improved by focusing rare follow-up resources on Fermi-LAT GRBs; as Nysewander et al. (2009) and McBreen et al. (2010) have shown, these events have brighter X-ray and optical afterglows on average, and are therefore accessible even for moderateaperture optical facilities. Targeting these bright afterglows will make it easier to measure the jet breaks, which have proven almost impossible to obtain in the Swift era. Finally, we note that one testable consequence of hyper-energetic GRBs is longlived afterglow emission ( $\gtrsim 1 \mathrm{yr}$ ). If the shock microphysics and the circumburst density do not undergo a drastic evolution, it should be possible to detect these afterglows with the upcoming generation of radio facilities and carry out calorimetry measurements.

We wish to thank D. A. Kann, R. Barniol Duran, P. Kumar, and the anonymous referee for valuable comments and discussions regarding this manuscript. We are grateful to the GROND team, in particular to S. McBreen and T. Krühler, for providing their observations of GRB 090323, GRB 090328, and GRB 090902B, and to A. Rau for assistance with the photometric calibration of the field of GRB 090926A.

S.B.C. and A.V.F. wish to acknowledge generous support from Gary and Cynthia Bengier, the Richard and Rhoda Goldman Fund, National Aeronautics and Space Administration (NASA)/Swift grants NNX09AL08G and NNX10AI21G, and National Science Foundation (NSF) grants AST-0607485 and AST-0908886. D.A.F. and F.A.H. were partially supported by NASA/Fermi Guest Investigator grants NNH07ZDA001N and NNH08ZDA001N. B.E.C. gratefully acknowledges support from an NSF Astronomy \& Astrophysics Postdoctoral Fellowship (AST-0802333). N.R.B. is supported through the Einstein Fellowship Program (NASA Cooperative Agreement NNG06DO90A). J.S.B. and his group were partially supported by NASA/Swift Guest Investigator grant NNX09AQ66G and a grant from DOE SciDAC.

P60 operations are funded in part by NASA through the Swift Guest Investigator Program (grant number NNG06GH61G). This work is based in part on observations obtained at the Gemini Observatory (Programs GS-2009A-Q-23, GS-2009B-Q-5, GN2009A-Q-26, and GN-2009B-Q-28), which is operated by the Association of Universities for Research in Astronomy, Inc., under a cooperative agreement with the NSF on behalf of the Gemini partnership: the NSF (US), the Particle Physics and Astronomy Research Council (UK), the National Research Council (Canada), CONICYT (Chile), the Australian Research Council (Australia), CNPq (Brazil), and CONICET (Argentina). We wish to thank the entire staff at Gemini for assistance with these observations. SMARTS is supported by NSF grant AST0707627.

Facilities: Fermi (LAT, GBM), VLA, PO:1.5m, Gemini: South (GMOS), Gemini:Gillett (GMOS), CTIO:2MASS (ANDICAM), Swift (XRT, UVOT)

\section{REFERENCES}

Abazajian, K. N., et al. 2009, ApJS, 182, 543

Abdo, A. A., et al. 2009a, ApJ, 706, L138

Abdo, A. A., et al. 2009b, Science, 323, 1688

Amati, L. 2006, MNRAS, 372, 233

Asano, K., Guiriec, S., \& Mészáros, P. 2009, ApJ, 705, L191 
Atwood, W. B., et al. 2009, ApJ, 697, 1071

Baldwin, J. A., \& Stone, R. P. S. 1984, MNRAS, 206, 241

Band, D., et al. 1993, ApJ, 413, 281

Barthelmy, S. D., et al. 2005, Space Sci. Rev., 120, 143

Beloborodov, A. M. 2010, MNRAS, 407, 1033

Berger, E., Kulkarni, S. R., \& Frail, D. A. 2003a, ApJ, 590, 379

Berger, E., Kulkarni, S. R., \& Frail, D. A. 2004, ApJ, 612, 966

Berger, E., Soderberg, A. M., Frail, D. A., \& Kulkarni, S. R. 2003b, ApJ, 587, L5

Berger, E., et al. 2001, ApJ, 556, 556

Berger, E., et al. 2005, ApJ, 634, 501

Birkl, R., Aloy, M. A., Janka, H.-T., \& Müller, E. 2007, A\&A, 463, 51

Bissaldi, E. 2009, GRB Coordinates Network, 9933, 1

Bissaldi, E. 2010, arXiv:1002.4194

Bissaldi, E., Briggs, M. S., Piron, F., Takahashi, H., \& Uehara, T. 2009, GRB Coordinates Network, 9972, 1

Bissaldi, E., \& Connaughton, V. 2009, GRB Coordinates Network, 9866, 1

Blandford, R. D., \& McKee, C. F. 1976, Phys. Fluids, 19, 1130

Blandford, R. D., \& Znajek, R. L. 1977, MNRAS, 179, 433

Bloom, J. S., Frail, D. A., \& Kulkarni, S. R. 2003, ApJ, 594, 674

Bloom, J. S., Frail, D. A., \& Sari, R. 2001, AJ, 121, 2879

Bošnjak, Ž., Daigne, F., \& Dubus, G. 2009, A\&A, 498, 677

Burenin, R., et al. 2009, GRB Coordinates Network, 9037, 1

Burrows, D. N., et al. 2005a, Science, 309, 1833

Burrows, D. N., et al. 2005b, Space Sci. Rev., 120, 165

Butler, N. R., Bloom, J. S., \& Poznanski, D. 2010, ApJ, 711, 495

Butler, N. R., \& Kocevski, D. 2007, ApJ, 663, 407

Butler, N. R., Kocevski, D., Bloom, J. S., \& Curtis, J. L. 2007, ApJ, 671, 656

Campana, S., et al. 2006, Nature, 442, 1008

Cavallo, G., \& Rees, M. J. 1978, MNRAS, 183, 359

Cenko, S. B., Bloom, J. S., Morgan, A. N., \& Perley, D. A. 2009, GRB Coordinates Network, 9053, 1

Cenko, S. B., \& Perley, D. A. 2009, GRB Coordinates Network, 9027, 1

Cenko, S. B., et al. 2006, PASP, 118, 1396

Cenko, S. B., et al. 2008, ApJ, 677, 441

Cenko, S. B., et al. 2010, ApJ, 711, 641

Chakrabarti, S. K., et al. 2009, GRB Coordinates Network, 10009, 1

Chandra, P., \& Frail, D. A. 2009, GRB Coordinates Network, 9889, 1

Chandra, P., et al. 2008, ApJ, 683, 924

Chandra, P., et al. 2010, ApJ, 712, L31

Chevalier, R. A., \& Li, Z.-Y. 1999, ApJ, 520, L29

Chevalier, R. A., \& Li, Z.-Y. 2000, ApJ, 536, 195

Chevalier, R. A., Li, Z.-Y., \& Fransson, C. 2004, ApJ, 606, 369

Chornock, R., Perley, D. A., Cenko, S. B., \& Bloom, J. S. 2009, GRB Coordinates Network, 9028, 1

Cobb, B. E., Bailyn, C. D., van Dokkum, P. G., Buxton, M. M., \& Bloom, J. S. 2004, ApJ, 608, L93

Cobb, B. E., Bailyn, C. D., van Dokkum, P. G., \& Natarajan, P. 2006, ApJ, 645, L113

Cucchiara, A., Fox, D. B., Tanvir, N., \& Berger, E. 2009, GRB Coordinates Network, 9873, 1

Curran, P. A., Starling, R. L. C., van der Horst, A. J., \& Wijers, R. A. M. J. 2009, MNRAS, 395, 580

Cutini, S., Vasileiou, V., \& Chiang, J. 2009, GRB Coordinates Network, 9077, 1

Daigne, F., \& Mochkovitch, R. 1998, MNRAS, 296, 275

Demorest, P. B., Pennucci, T., Ransom, S. M., Roberts, M. S. E., \& Hessels, J. W. T. 2010, Nature, 467, 1081

de Palma, F., Bregeon, J., \& Tajima, H. 2009, GRB Coordinates Network, 9867, 1

de Ugarte Postigo, A., Xu, D., Malesani, D., Hjorth, J., Fynbo, J. P. U., Jakobsson, P., \& Adamo, A. 2009, GRB Coordinates Network, 9051, 1

Djorgovski, S. G., et al. 1997, Nature, 387, 876

Evans, P. A. 2009, GRB Coordinates Network, 9871, 1

Falcone, A. D., et al. 2007, ApJ, 671, 1921

Ferrero, P., et al. 2006, A\&A, 457, 857

Filippenko, A. V. 1997, ARA\&A, 35, 309

Frail, D. A., Chandra, P., \& Cenko, B. 2009, GRB Coordinates Network, 9060, 1

Frail, D. A., Soderberg, A. M., Kulkarni, S. R., Berger, E., Yost, S., Fox, D. W., \& Harrison, F. A. 2005, ApJ, 619, 994

Frail, D. A., Waxman, E., \& Kulkarni, S. R. 2000a, ApJ, 537, 191

Frail, D. A., et al. 2000b, ApJ, 538, L129

Frail, D. A., et al. 2001, ApJ, 562, L55

Frail, D. A., et al. 2003, ApJ, 590, 992

Frail, D. A., et al. 2006, ApJ, 646, L99

Freedman, D. L., \& Waxman, E. 2001, ApJ, 547, 922
Fukugita, M., Shimasaku, K., \& Ichikawa, T. 1995, PASP, 107, 945

Galama, T. J., et al. 1998, Nature, 395, 670

Gal-Yam, A., et al. 2004, ApJ, 609, L59

Gehrels, N., Ramirez-Ruiz, E., \& Fox, D. B. 2009, ARA\&A, 47, 567

Gehrels, N., et al. 2004, ApJ, 611, 1005

Ghisellini, G., Ghirlanda, G., Nava, L., \& Celotti, A. 2010, MNRAS, 403, 926

Giuliani, A., et al. 2008, A\&A, 491, L25

Golenetskii, S., et al. 2009a, GRB Coordinates Network, 9049, 1

Golenetskii, S., et al. 2009b, GRB Coordinates Network, 9959, 1

Golenetskii, S., et al. 2009c, GRB Coordinates Network, 9030, 1

Golenetskii, S., et al. 2009d, GRB Coordinates Network, 9050, 1

González, M. M., Dingus, B. L., Kaneko, Y., Preece, R. D., Dermer, C. D., \& Briggs, M. S. 2003, Nature, 424, 749

Granot, J. 2007, RevMexAA Conf. Ser., 27, 140

Granot, J., \& Sari, R. 2002, ApJ, 568, 820

Greiner, J., et al. 2008, PASP, 120, 405

Greiner, J., et al. 2009, A\&A, 498, 89

Grupe, D., et al. 2007, ApJ, 662, 443

Grupe, D., et al. 2010, ApJ, 711, 1008

Guetta, D., \& Della Valle, M. 2007, ApJ, 657, L73

Guidorzi, C., et al. 2009, GRB Coordinates Network, 9039, 1

Haislip, J., et al. 2009a, GRB Coordinates Network, 9953, 1

Haislip, J., et al. 2009b, GRB Coordinates Network, 9937, 1

Haislip, J., et al. 2009c, GRB Coordinates Network, 9984, 1

Haislip, J., et al. 2009d, GRB Coordinates Network, 0003, 1

Haislip, J., et al. 2009e, GRB Coordinates Network, 9982, 1

Harikae, S., Kotake, K., \& Takiwaki, T. 2010, ApJ, 713, 304

Harrison, F., Cenko, B., Frail, D. A., Chandra, P., \& Kulkarni, S. 2009, GRB Coordinates Network, 9043, 1

Harrison, F. A., et al. 2001, ApJ, 559, 123

Hook, I. M., Jørgensen, I., Allington-Smith, J. R., Davies, R. L., Metcalfe, N., Murowinski, R. G., \& Crampton, D. 2004, PASP, 116, 425

Hurley, K., Briggs, M. S., Kippen, R. M., Kouveliotou, C., Meegan, C., Fishman, G., Cline, T., \& Boer, M. 1999, ApJS, 120, 399

Hurley, K., Goldsten, J., von Kienlin, A., Lichti, G., Rau, A., Connaughton, V., Briggs, M., \& Meegan, C. 2009, GRB Coordinates Network, 9023, 1

Hurley, K., et al. 1994, Nature, 372, 652

Iwamoto, K., et al. 1998, Nature, 395, 672

Jakobsson, P., et al. 2006, A\&A, 447, 897

Jordi, K., Grebel, E. K., \& Ammon, K. 2006, A\&A, 460, 339

Kann, D. A., Klose, S., \& Zeh, A. 2006, ApJ, 641, 993

Kann, D. A., Laux, U., Ludwig, F., \& Stecklum, S. 2009a, GRB Coordinates Network, 9063, 1

Kann, D. A., Laux, U., \& Stecklum, S. 2009b, GRB Coordinates Network, 9033, 1

Kann, D. A., Laux, U., \& Stecklum, S. 2009c, GRB Coordinates Network, 9041, 1

Kann, D. A., et al. 2010, ApJ, 720, 1513

Kennea, J. 2009, GRB Coordinates Network, 9045, 1

Kennea, J., Evans, P., \& Goad, M. 2009a, GRB Coordinates Network, 9024, 1

Kennea, J., Evans, P., \& Goad, M. 2009b, GRB Coordinates Network, 9046, 1

Kennea, J. A., \& Stratta, G. 2009, GRB Coordinates Network, 9868, 1

Kobayashi, S., Piran, T., \& Sari, R. 1997, ApJ, 490, 92

Kocevski, D., \& Butler, N. 2008, ApJ, 680, 531

Komissarov, S. S., Vlahakis, N., \& Königl, A. 2010, MNRAS, 407, 17

Kulkarni, S. R., et al. 1998, Nature, 395, 663

Kulkarni, S. R., et al. 1999, ApJ, 522, L97

Kumar, P., \& Barniol Duran, R. 2009, MNRAS, 400, L75

Kumar, P., \& Barniol Duran, R. 2010, MNRAS, 409, 226

Kumar, P., \& Piran, T. 2000, ApJ, 535, 152

Landolt, A. U. 1992, AJ, 104, 340

Lazzati, D., Rossi, E., Covino, S., Ghisellini, G., \& Malesani, D. 2002, A\&A, 396, L5

Lee, H. K., Wijers, R. A. M. J., \& Brown, G. E. 2000, Phys. Rep., 325, 83

Li, W., Jha, S., Filippenko, A. V., Bloom, J. S., Pooley, D., Foley, R. J., \& Perley, D. A. 2006, PASP, 118, 37

Li, Z.-Y., \& Chevalier, R. A. 1999, ApJ, 526, 716

Liang, E., Zhang, B., Virgili, F., \& Dai, Z. G. 2007, ApJ, 662, 1111

Liang, E.-W., Racusin, J. L., Zhang, B., Zhang, B.-B., \& Burrows, D. N. 2008, ApJ, 675, 528

Lithwick, Y., \& Sari, R. 2001, ApJ, 555, 540

MacFadyen, A. I., \& Woosley, S. E. 1999, ApJ, 524, 262

MacFadyen, A. I., Woosley, S. E., \& Heger, A. 2001, ApJ, 550, 410

Maeda, K., et al. 2007, ApJ, 658, L5

Malesani, D., et al. 2004, ApJ, 609, L5

Malesani, D., et al. 2009, GRB Coordinates Network, 9942, 1 
Matheson, T., Filippenko, A. V., Ho, L. C., Barth, A. J., \& Leonard, D. C. 2000, AJ, 120, 1499

Mazzali, P. A., et al. 2006, Nature, 442, 1018

McBreen, S., et al. 2010, A\&A, 516, A71

McEnery, J., Cutini, S., Ohno, M., \& Koerding, E. 2009, GRB Coordinates Network, 9044, 1

McKinney, J. C. 2005, ApJ, 630, L5

Meegan, C., et al. 2009, ApJ, 702, 791

Meszaros, P., \& Rees, M. J. 1997, ApJ, 476, 232

Mészáros, P., \& Rees, M. J. 2000, ApJ, 530, 292

Metzger, B. D., Thompson, T. A., \& Quataert, E. 2007, ApJ, 659, 561

Mirabal, N., Halpern, J. P., An, D., Thorstensen, J. R., \& Terndrup, D. M. 2006, ApJ, 643, L99

Modjaz, M., et al. 2006, ApJ, 645, L21

Modjaz, M., et al. 2008, AJ, 135, 1136

Moin, A., Frail, D. A., Tingay, S., \& Macquart, J. 2009, GRB Coordinates Network, 10020, 1

Molinari, E., et al. 2007, A\&A, 469, L13

Monet, D. G., et al. 2003, AJ, 125, 984

Nakar, E., \& Granot, J. 2007, MNRAS, 380, 1744

Narayan, R., Piran, T., \& Kumar, P. 2001, ApJ, 557, 949

Noda, K., et al. 2009, GRB Coordinates Network, 9951, 1

Nysewander, M., Fruchter, A. S., \& Pe'er, A. 2009, ApJ, 701, 824

Oates, S. R. 2009, GRB Coordinates Network, 9048, 1

Ohno, M., Cutini, S., McEnery, J., Chiang, J., \& Koerding, E. 2009, GRB Coordinates Network, 9021,1

Oke, J. B. 1990, AJ, 99, 1621

Oke, J. B., \& Gunn, J. E. 1983, ApJ, 266, 713

Panaitescu, A. 2005, MNRAS, 363, 1409

Panaitescu, A. 2006, MNRAS, 367, L42

Panaitescu, A. 2007, MNRAS, 380, 374

Panaitescu, A., \& Kumar, P. 2000, ApJ, 543, 66

Panaitescu, A., \& Kumar, P. 2001a, ApJ, 560, L49

Panaitescu, A., \& Kumar, P. 2001b, ApJ, 554, 667

Panaitescu, A., \& Kumar, P. 2002, ApJ, 571, 779

Pandey, S. B., et al. 2010, ApJ, 714, 799

Pei, Y. C. 1992, ApJ, 395, 130

Perley, D. A. 2009, GRB Coordinates Network, 9042, 1

Perley, D. A., Klein, C. R., Morgan, A. N., \& Petigura, E. 2009a, GRB Coordinates Network, 9036, 1

Perley, D. A., et al. 2008, ApJ, 688, 470

Perley, D. A., et al. 2009b, ApJ, 696, 1871

Perley, R., et al. 2009c, Proc. IEEE, 97, 1448

Perna, R., Sari, R., \& Frail, D. 2003, ApJ, 594, 379

Pian, E., et al. 2006, Nature, 442, 1011

Piran, T. 2005, Rev. Mod. Phys., 76, 1143

Piran, T., \& Nakar, E. 2010, ApJ, 718, L63

Piron, F., et al. 2009, in 2009 Fermi Symposium, ed. eConf Proceedings C0911022

Popham, R., Woosley, S. E., \& Fryer, C. 1999, ApJ, 518, 356

Price, P. A., et al. 2002, ApJ, 572, L51

Racusin, J. L., et al. 2009, ApJ, 698, 43

Rau, A., Connaughton, V., \& Briggs, M. 2009, GRB Coordinates Network, 9057, 1

Rau, A., et al. 2010, ApJ, 720, 862

Razzaque, S., Dermer, C. D., \& Finke, J. D. 2010, Open Astronomy Journal, 3, 150

Rees, M. J., \& Meszaros, P. 1998, ApJ, 496, L1

Reichart, D., et al. 2005, Il Nuovo Cimento C, 28, 767

Rhoads, J. E. 1999, ApJ, 525, 737

Roming, P. W. A., et al. 2005, Space Sci. Rev., 120, 95

Rosswog, S., Ramirez-Ruiz, E., \& Davies, M. B. 2003, MNRAS, 345, 1077

Ruffert, M., \& Janka, H.-T. 1999, A\&A, 344, 573

Rumyantsev, V., \& Pozanenko, A. 2009, GRB Coordinates Network, 9324, 1

Ryde, F. 2004, ApJ, 614, 827
Ryde, F., et al. 2010, ApJ, 709, L172

Sari, R., \& Esin, A. A. 2001, ApJ, 548, 787

Sari, R., \& Piran, T. 1999, ApJ, 517, L109

Sari, R., Piran, T., \& Halpern, J. P. 1999, ApJ, 519, L17

Sari, R., Piran, T., \& Narayan, R. 1998, ApJ, 497, L17

Sazonov, S. Y., Lutovinov, A. A., \& Sunyaev, R. A. 2004, Nature, 430, 646

Schady, P., et al. 2007, MNRAS, 380, 1041

Schlegel, D. J., Finkbeiner, D. P., \& Davis, M. 1998, ApJ, 500, 525

Shen, R., Kumar, P., \& Robinson, E. L. 2006, MNRAS, 371, 1441

Skrutskie, M. F., et al. 2006, AJ, 131, 1163

Soderberg, A. M., \& Ramirez-Ruiz, E. 2003, MNRAS, 345, 854

Soderberg, A. M., et al. 2004, Nature, 430, 648

Soderberg, A. M., et al. 2006, Nature, 442, 1014

Soderberg, A. M., et al. 2010, Nature, 463, 513

Sollerman, J., et al. 2006, A\&A, 454, 503

Spergel, D. N., et al. 2007, ApJS, 170, 377

Starling, R. L. C., van der Horst, A. J., Rol, E., Wijers, R. A. M. J., Kouveliotou,

C., Wiersema, K., Curran, P. A., \& Weltevrede, P. 2008, ApJ, 672, 433

Starling, R. L. C., et al. 2009, MNRAS, 400, 90

Stone, R. P. S., \& Baldwin, J. A. 1983, MNRAS, 204, 347

Stratta, G., D’Elia, V., \& Perri, M. 2009, GRB Coordinates Network, 9876, 1

Swenson, C. A., \& Siegel, M. H. 2009, GRB Coordinates Network, 9869, 1

Swenson, C. A., \& Stratta, G. 2009, GRB Coordinates Network, 9877, 1

Swenson, C. A., et al. 2010, ApJ, 718, L14

Tam, P. H., Pun, C. S. J., Huang, Y. F., \& Cheng, K. S. 2005, New Astron., 10, 535

Tchekhovskoy, A., McKinney, J. C., \& Narayan, R. 2009, ApJ, 699, 1789

Tchekhovskoy, A., Narayan, R., \& McKinney, J. C. 2010, New Astron., 15, 749

Terada, Y., et al. 2009, GRB Coordinates Network, 9897, 1

Thompson, T. A., Chang, P., \& Quataert, E. 2004, ApJ, 611, 380

Thomsen, B., et al. 2004, A\&A, 419, L21

Uehara, T., Takahashi, H., \& McEnery, J. 2009, GRB Coordinates Network, 9934, 1

Updike, A. C., Filgas, R., Kruehler, T., Greiner, J., \& McBreen, S. 2009, GRB Coordinates Network, 9026, 1

Usov, V. V. 1992, Nature, 357, 472

van der Horst, A. J. 2009a, GRB Coordinates Network, 9035, 1

van der Horst, A. J. 2009b, GRB Coordinates Network, 9047, 1

van der Horst, A. J., Kamble, A. P., Wijers, R. A. M. J., \& Kouveliotou, C. 2009, GRB Coordinates Network, 9883, 1

van der Horst, A. J., et al. 2008, A\&A, 480, 35

van Eerten, H., Zhang, W., \& MacFadyen, A. 2010, ApJ, 722, 235

van Eerten, H. J., Meliani, Z., Wijers, R. A. M. J., \& Keppens, R. 2011, MNRAS, 410, 2016

van Haarlem, M. P. 2005, in Radio Astronomy from Karl Jansky to Microjansky, ed. L. I. Gurvits, S. Frey, \& S. Rawlings (EAS Pub. Ser. 15; Les Ulis: EDP Science), 431

Vetere, L. 2009, GRB Coordinates Network, 9961, 1

Vetere, L., Evans, P. A., \& Goad, M. R. 2009, GRB Coordinates Network, 9936, 1

Wade, R. A., \& Horne, K. 1988, ApJ, 324, 411

Wang, X. F., Xin, L. P., Zheng, W. K., Qiu, Y. L., Wei, J. Y., Deng, J. S., \& Hu, J. Y. 2009, GRB Coordinates Network, 9034, 1

Waxman, E., Kulkarni, S. R., \& Frail, D. A. 1998, ApJ, 497, 288

Wijers, R. A. M. J., \& Galama, T. J. 1999, ApJ, 523, 177

Willingale, R., et al. 2007, ApJ, 662, 1093

Woosley, S. E. 1993, ApJ, 405, 273

Woosley, S. E., \& Bloom, J. S. 2006, ARA\&A, 44, 507

Woosley, S. E., \& Heger, A. 2006, ApJ, 637, 914

Yost, S. A., Harrison, F. A., Sari, R., \& Frail, D. A. 2003, ApJ, 597, 459

Yost, S. A., et al. 2002, ApJ, 577, 155

Zeh, A., Klose, S., \& Kann, D. A. 2006, ApJ, 637, 889

Zhang, W., \& MacFadyen, A. 2009, ApJ, 698, 1261

Zhang, W., Woosley, S. E., \& MacFadyen, A. I. 2003, ApJ, 586, 356 